\title{
Chandra Multiwavelength Project X\#Ray Point Source Catalog
}

\section{Citation}

Kim, Minsun, Dong\#Woo Kim, Belinda J. Wilkes, Paul J. Green, Eunhyeuk Kim, Craig S. Anderson, Wayne A. Barkhouse, et al. 2007. "Chandra Multiwavelength Project X\#Ray Point Source Catalog." The Astrophysical Journal Supplement Series 169 (2) (April): 401-429. doi:10.1086/511634.

\section{Published Version}

doi:10.1086/511634

\section{Permanent link}

http://nrs.harvard.edu/urn-3:HUL.InstRepos:29921872

\section{Terms of Use}

This article was downloaded from Harvard University's DASH repository, and is made available under the terms and conditions applicable to Other Posted Material, as set forth at http:// nrs.harvard.edu/urn-3:HUL.InstRepos:dash.current.terms-of-use\#LAA

\section{Share Your Story}

The Harvard community has made this article openly available.

Please share how this access benefits you. Submit a story.

\section{Accessibility}




\title{
CHANDRA MULTIWAVELENGTH PROJECT X-RAY POINT SOURCE CATALOG
}

\author{
Minsun Kim, ${ }^{1,2}$ Dong-Woo Kim, ${ }^{1}$ Belinda J. Wilkes, ${ }^{1}$ Paul J. Green, ${ }^{1}$ Eunhyeuk Kim, ${ }^{1}$ Craig S. Anderson, ${ }^{1}$

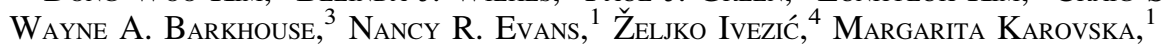 \\ Vinay L. Kashyap, ${ }^{1}$ Myung Gyoon Lee, ${ }^{2}$ Peter Maksym, ${ }^{5}$ Amy E. Mossman, ${ }^{1}$ \\ John D. Silverman, ${ }^{6}$ and Harvey D. Tananbaum ${ }^{1}$ \\ Received 2006 April 20; accepted 2006 November 20
}

\begin{abstract}
We present the Chandra Multiwavelength Project (ChaMP) X-ray point source catalog with $\sim 6800$ X-ray sources detected in 149 Chandra observations covering $\sim 10 \mathrm{deg}^{2}$. The full ChaMP catalog sample is 7 times larger than the initial published ChaMP catalog. The exposure time of the fields in our sample ranges from 0.9 to $124 \mathrm{ks}$, corresponding to a deepest X-ray flux limit of $f_{0.5-8.0}=9 \times 10^{-16} \mathrm{ergs} \mathrm{cm}^{-2} \mathrm{~s}^{-1}$. The ChaMP X-ray data have been uniformly reduced and analyzed with ChaMP-specific pipelines and then carefully validated by visual inspection. The ChaMP catalog includes X-ray photometric data in eight different energy bands as well as X-ray spectral hardness ratios and colors. To best utilize the ChaMP catalog, we also present the source reliability, detection probability, and positional uncertainty. To quantitatively assess those parameters, we performed extensive simulations. In particular, we present a set of empirical equations: the flux limit as a function of effective exposure time and the positional uncertainty as a function of source counts and off-axis angle. The false source detection rate is $\sim 1 \%$ of all detected ChaMP sources, while the detection probability is better than $\sim 95 \%$ for sources with counts $\gtrsim 30$ and off-axis angle $<5^{\prime}$. The typical positional offset between ChaMP X-ray source and their SDSS optical counterparts is $0.7^{\prime \prime} \pm 0.4^{\prime \prime}$, derived from $\sim 900$ matched sources.
\end{abstract}

Subject headings: catalogs — surveys - X-rays: general

Online material: color figures, machine-readable tables

\section{INTRODUCTION}

Since the cosmic X-ray background (CXRB) was discovered by Giacconi et al. (1962) there have been several X-ray missions, such as Einstein, ROSAT, ASCA, and BeppoSAX. Chandra and $X M M$-Newton are the current powerful X-ray missions with more sensitive imaging spectroscopy and higher positional accuracy than previous missions. To investigate the formation and evolution of galaxies, clusters of galaxies, and the large-scale structure of the universe, these previous and current X-ray missions have provided several deep and wide extragalactic X-ray surveys (see Brandt \& Hasinger 2005 and references therein for a detailed review). Bauer et al. (2004) established the X-ray number counts from the Chandra Deep Field-North (CDF-N) and South (CDF-S) and found that $\sim 90 \%(\sim 93 \%)$ of the CXRB is resolved into discrete X-ray sources in the $0.5-2 \mathrm{keV}(2-8 \mathrm{keV})$ band. Using the $X M M-N e w t o n$ observation of the Lockman Hole and the CDFs, Worsley et al. (2005) found that the resolved fractions of the CXRB are $\sim 85 \%(0.5-2 \mathrm{keV}), \sim 80 \%(2-10 \mathrm{keV})$, and $\sim 50 \%$ ( $\gtrsim 8 \mathrm{keV}$ ), respectively. Therefore, the CXRB is predominantly resolved into discrete sources in the $0.5-2$ and $2-10 \mathrm{keV}$ bands; however, the constituents of the CXRB in the hard energy band $(\gtrsim 8 \mathrm{keV})$ are still unknown.

\footnotetext{
${ }^{1}$ Harvard-Smithsonian Center for Astrophysics, Cambridge, MA 02138; mkim@cfa.harvard.edu.

2 Department of Physics and Astronomy, Astronomy Program, Seoul National University, Seoul, Korea.

3 Department of Astronomy, University of Illinois at Urbana-Champaign, Urbana, IL 61801.

4 Department of Astronomy, University of Washington, Seattle, WA 98195.

5 Department of Physics and Astronomy, Northwestern University, Evanston, IL 60208

${ }^{6}$ Max-Planck-Institut für extraterrestrische Physik, D-84571 Garching, Germany.
}

The deepest X-ray surveys are the 2 Ms CDF-N (Brandt et al. 2001; Alexander et al. 2003) and the 1 Ms CDF-S (Giacconi et al. 2001; Rosati et al. 2002) covering small sky areas $\left(\sim 0.12 \mathrm{deg}^{2}\right)$. The faint flux limits of the CDFs are $\sim 2 \times 10^{-17} \mathrm{erg} \mathrm{cm}^{-2} \mathrm{~s}^{-1}$ $(0.5-2 \mathrm{keV})$ and $\sim 2 \times 10^{-16} \mathrm{erg} \mathrm{cm}^{-2} \mathrm{~s}^{-1}(2-8 \mathrm{keV})$, respectively. The XMM-Newton survey of the Lockman Hole covers $\sim 0.43 \mathrm{deg}^{2}$ with an effective exposure time of $\sim 700 \mathrm{ks}$ (flux range of $\sim$ few $\times 10^{-16} \sim(3-5) \times 10^{-14} \mathrm{erg} \mathrm{cm}^{-2} \mathrm{~s}^{-1}$ in each band) (Worsley et al. 2004). The XMM-Newton large-scale structure survey $(X M M$-LSS $)$ is a medium depth $(\sim 10 \mathrm{ks})$ and large area $\left(\sim 64 \mathrm{deg}^{2}\right) \mathrm{X}$-ray survey (Pierre et al. 2004). The $3 \mathrm{deg}^{2}$ $X M M$-Newton medium depth survey (XMDS) pointed at the center of $X M M-$ LSS reaches a flux limit of $\sim 10^{-15} \mathrm{erg} \mathrm{cm}^{-2} \mathrm{~s}^{-1}$ in the 0.5-2 keV band (Chiappetti et al. 2005). The Chandra X-ray survey of the NDWFS Bootes is a contiguous wide $\left(\sim 9.3 \mathrm{deg}^{2}\right)$ and medium depth $(\sim 5 \mathrm{ks})$ survey and contains 4642 sources with flux limits of $4 \times 10^{-15} \mathrm{erg} \mathrm{cm}^{-2} \mathrm{~s}^{-1}$ in the $0.5-7 \mathrm{keV}$ band (Murray et al. 2005).

The Chandra Multiwavelength Project (ChaMP) is a serendipitous Chandra archival survey of X-ray sources covering a wide area $\left(\sim 10 \mathrm{deg}^{2}\right)$ with a range of depths $0.9-124$ ks exposures. The main scientific goals of the ChaMP are to investigate the (1) formation and evolution of high-redshift active galactic nuclei (AGNs), (2) properties of X-ray luminous galaxies and clusters, and (3) constituents of the CXRB. Kim et al. (2004a, hereafter Paper I) reported the first ChaMP X-ray source catalog including 991 near on-axis, bright X-ray sources obtained from 62 ChaMP fields, and Green et al. (2004) have performed optical and spectroscopic follow-up observations of a subset of these ChaMP $\mathrm{X}$-ray sources.

Using the first ChaMP X-ray point source catalog and the follow-up surveys of optical and spectroscopic observations, there have been several interesting results. Kim et al. (2004b) established 
the number counts of the ChaMP X-ray point sources in the $0.5-2$ and $2-8 \mathrm{keV}$ bands, which agreed with previous studies within the uncertainties, and found that there are no significant field-to-field variations in cosmic X-ray source number density on the scale of $\sim 16^{\prime}$, which corresponds to a single Chandra observational field of view. Silverman et al. (2005a) found the turnover in the comoving space density of X-ray selected, luminous type 1 AGNs $\left(\log L_{\mathrm{X}}>44.5 \mathrm{erg} \mathrm{s}^{-1}\right.$ measured in the $0.3-8 \mathrm{keV}$ band) to be at $z>2.5$, consistent with the optical results. The hard X-rayemitting AGNs in 20 ChaMP fields were investigated and classified as broad emission-line AGNs (62\%), narrow emission-line galaxies $(24 \%)$, absorption line galaxies $(7 \%)$, stars $(5 \%)$, or clusters $(2 \%)$. Most X-ray-unabsorbed AGNs $\left(N_{\mathrm{H}}<10^{22} \mathrm{~cm}^{-2}\right)$ have broad emission lines and blue optical colors, but there is a significant population of redder AGNs with broad optical emission lines. Most X-ray-absorbed AGNs $\left(10^{22} \mathrm{~cm}^{-2}<N_{\mathrm{H}}<10^{24} \mathrm{~cm}^{-2}\right)$ are associated with narrow emission-line galaxies, those with red optical colors being dominated by luminous, early-type galaxy hosts rather than dust-reddened AGNs (Silverman et al. 2005b).

Barkhouse et al. (2006) presented the ChaMP X-ray extended source catalog, which contains 55 extended sources from 130 Chandra fields. From the overlapping optical/X-ray fields $\left(6.1 \mathrm{deg}^{2}\right)$ they found 115 optical cluster candidates, of which 13 were detected as extended X-ray sources. A comparison of the richness of the optical-only versus X-ray/optically matched cluster samples shows that the average richness of the optical-only clusters is smaller by $\sim 4 \sigma$ than the matched X-ray/optical clusters. This result suggests that the optical-only sample is either (1) composed of mainly poor systems that lack sufficient hot gas for detection in the X-rays, or (2) are contaminated by nonvirialized filaments associated with the large-scale structure. Kim et al. (2006a) investigated the normal galaxies at intermediate redshift in the ChaMP fields and found that normal galaxies at redshift $z<0.1$ do not show significant evolution in $L_{X} / L_{B}$. They built cumulative number counts and luminosity functions of the normal galaxies, and they found that a group of NELGs appear to be heavily obscured in X-rays, while the low-redshift AGNs in this sample do not appear to be significantly absorbed. Also, they found two $\mathrm{E}+\mathrm{A}$ galaxy candidates, and they concluded that those galaxies support the merger/interaction scenario of galaxy formation from their X-ray spectra studies.

In this paper, we present the ChaMP X-ray point source catalog including $\sim 6800 \mathrm{X}$-ray point sources obtained from 149 ChaMP fields and covering a sky area of $\sim 10 \mathrm{deg}^{2}$. Compared to the first ChaMP X-ray point source catalog, this catalog contains 7 times more sources, covers 3 times more sky area, and includes fainter sources and those with larger off-axis angles. We performed extensive simulations to investigate the sensitivity, source probability, and positional uncertainty of the ChaMP sources. This catalog allows more statistically robust results from X-ray point source studies. The ChaMP data reduction procedures are similar to those in Paper I, therefore, we briefly summarize and/or skip those parts already described in Paper I and concentrate on newly added or improved procedures. In $\S 2$, we summarize the selection criteria and properties of the ChaMP fields. In $\S 3$, the data reduction and analysis of the ChaMP are described. Section 4 discusses the process and results of the ChaMP simulations. The ChaMP X-ray point source catalogs are provided in $\S 5$, and a summary and conclusions are given in $\S 6$.

\section{ChaMP FIELD SELECTION}

We selected Chandra fields observed with ACIS at high Galactic latitude, $|b|>20^{\circ}$ and excluded those fields containing large extended sources, planetary observations, and local group galaxies. Fields intended by their PIs as surveys were also excluded (see Paper I). These selection criteria yield 149 ChaMP fields in Chandra cycles 1 and 2, consisting of 35 ACIS-I and 114 ACIS-S observations. Seven ACIS-I and 28 ACIS-S ChaMP fields partly overlap one another on the sky, and those sources detected in multiple observations are listed separately, for example, to allow the study of source variability. In Table 1, the observational parameters of 149 ChaMP fields are listed in order of right ascension.

In Figure 1, we display the 149 ChaMP field locations in equatorial coordinates. Red circles represent ACIS-I at the aim point, blue circles ACIS-S.

The circle size crudely indicates the Chandra exposure time, ranging from 0.9 to $124 \mathrm{ks}$. The ChaMP samples are uniformly distributed over the entire celestial sphere except (by selection) the Galactic plane region. Figure 2 shows the number distributions of the exposure times and Galactic extinction of the ChaMP fields in top and bottom panels, respectively. The mean exposure time of the ChaMP is $\sim 25 \mathrm{ks}$ and the mean Galactic extinction is $N_{\mathrm{H}}=(3.4 \pm 2.2) \times 10^{20} \mathrm{~cm}^{-2}$. The ChaMP samples cover a wide range of exposure times, and the Galactic extinction of the ChaMP fields are generally much lower than those of Galactic plane $\left(N_{\mathrm{H}} \sim 10^{22}-10^{23} \mathrm{~cm}^{-2}\right)$. The 62 ChaMP fields included in Paper I are represented by shaded histograms. In this study, the X-ray point source catalog includes all X-ray sources in 149 ChaMP fields as well as fainter and larger off-axis angle sources, while the catalog in Paper I included only near on-axis (off-axis angle $<6^{\prime}$ or S3 chip for ACIS-S observations) and bright (net counts $>20$ ) sources in 62 ChaMP fields.

\section{ChaMP DATA REDUCTION}

We have developed a ChaMP-specific pipeline, XPIPE, to reduce the Chandra data. The pipeline consists of three main parts; (1) data correction and data screening using the $\mathrm{CIAO}^{7}$ package, (2) source detection using the wavdetect tool in the CIAO package, and (3) source extraction using the xapphot tool (E. Kim et al. 2007, in peparation; Martini et al. 2006) based on the cfitsio 8 library. The data correction and data screening procedures are the same as in Paper I. We do not use sources detected in the S4 chip (CCDID 8) because of the streaking problem (see Paper I).

\subsection{Source Detection}

For source detection, we use the wavdetect tool available in the CIAO package. The wavdetect tool consists of two parts: wtransform, convolving the data with the wavelet function for selected size scales; and wrecon, constructing a final source list and estimating various parameters for each source (Freeman et al. 2002). We run wavdetect in the B band (see Table 2 for energy band definition) with a significance threshold parameter of $10^{-6}$, which corresponds to one possible spurious pixel in one CCD (see $\S 4.3 .2$ for our simulation results on the probability of finding a spurious source). We select a range of scale size parameters in seven steps from 1 to 64 pixels $\left(1\right.$ pixel $\left.=0.492^{\prime \prime}\right)$. To avoid finding spurious sources located at the edge of the CCD chips, a minimum of $10 \%$ of the on-axis exposure was required for source detection. Exposure maps of the ChaMP fields were generated for each CCD at an energy of $1.5 \mathrm{keV}$ with an appropriate aspect histogram. ${ }^{9}$ Other parameters were set at the default values given in wavdetect. The positions provided by wavdetect in CIAO version 2.3 for off-axis sources, where the shape of the point-spread

\footnotetext{
7 See http://cxc.harvard.edu/ciao.

8 See http://heasarc.gsfc.nasa.gov/docs/software/fitsio/fitsio.html.

9 See http://cxc.harvard.edu/ciao/threads/expmap_acis_single.
} 
TABLE 1

List of ChaMP FIELDS

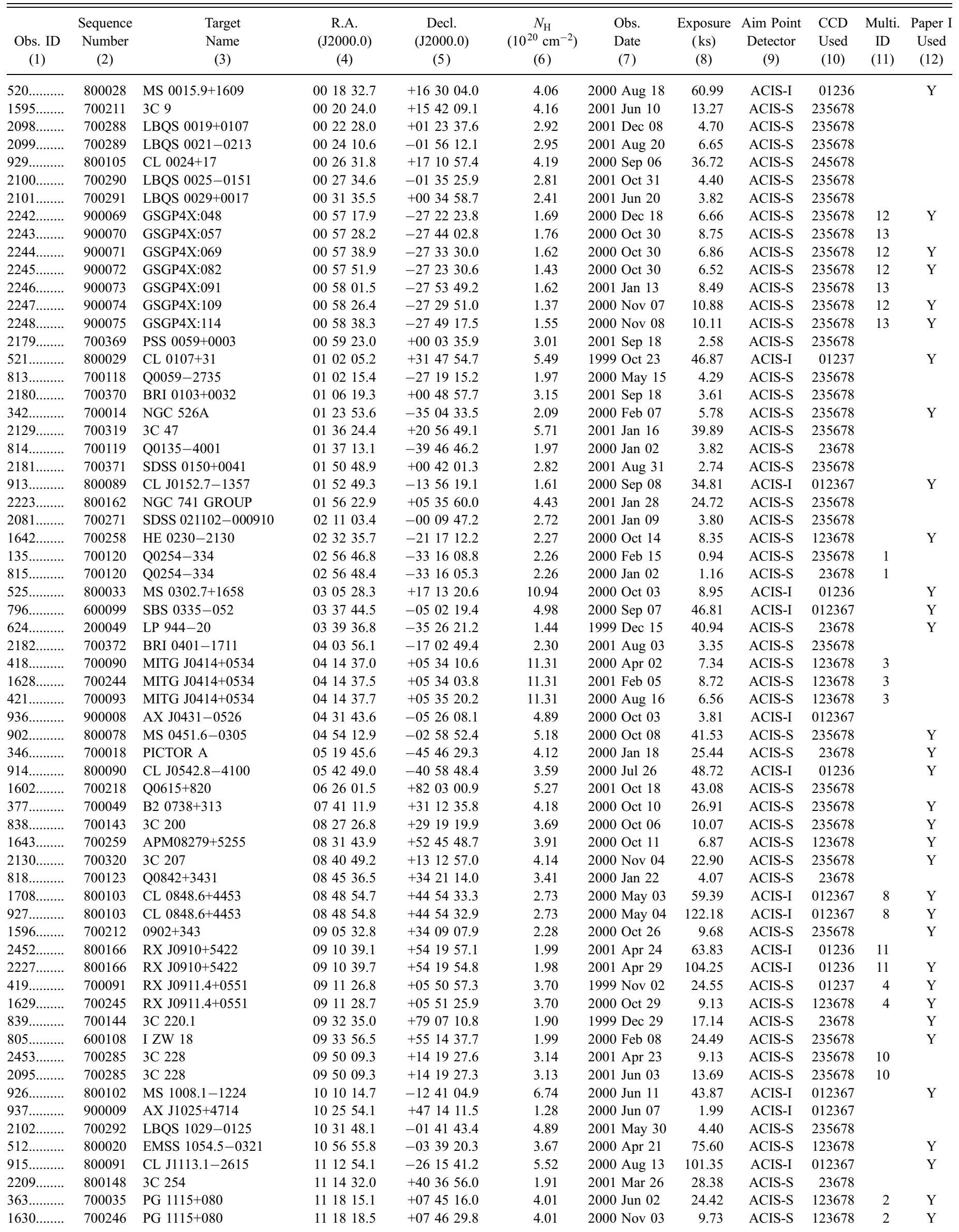


TABLE 1 - Continued

\begin{tabular}{|c|c|c|c|c|c|c|c|c|c|c|c|}
\hline $\begin{array}{c}\text { Obs. ID } \\
\text { (1) }\end{array}$ & $\begin{array}{c}\text { Sequence } \\
\text { Number } \\
\text { (2) }\end{array}$ & $\begin{array}{l}\text { Target } \\
\text { Name } \\
(3)\end{array}$ & $\begin{array}{l}\text { R.A. } \\
(\mathrm{J} 2000.0) \\
(4)\end{array}$ & $\begin{array}{c}\text { Decl. } \\
(\mathrm{J} 2000.0) \\
(5)\end{array}$ & $\begin{array}{c}N_{\mathrm{H}} \\
\left(10^{20} \mathrm{~cm}^{-2}\right) \\
(6)\end{array}$ & $\begin{array}{l}\text { Obs. } \\
\text { Date } \\
(7)\end{array}$ & $\begin{array}{c}\text { Exposure } \\
(\mathrm{ks}) \\
(8)\end{array}$ & $\begin{array}{l}\text { Aim Point } \\
\text { Detector } \\
\text { (9) }\end{array}$ & $\begin{array}{l}\text { CCD } \\
\text { Used } \\
(10)\end{array}$ & $\begin{array}{l}\text { Multi. } \\
\text { ID } \\
\text { (11) }\end{array}$ & $\begin{array}{c}\text { Paper I } \\
\text { Used } \\
\text { (12) }\end{array}$ \\
\hline $868 \ldots$ & 700173 & PG $1115+407$ & 111842.8 & +402517.6 & 1.91 & 2000 Oct 03 & 17.37 & ACIS-I & 012367 & & $\mathrm{Y}$ \\
\hline $1660 \ldots \ldots \ldots \ldots \ldots$ & 800128 & V $1121+2327$ & 112048.5 & +232409.1 & 1.30 & 2001 Apr 23 & 68.19 & ACIS-I & 01236 & & \\
\hline $819 \ldots \ldots \ldots \ldots \ldots$ & 700124 & UM 425 & 112319.6 & +013717.7 & 4.09 & 2000 Apr 07 & 2.55 & ACIS-S & 235678 & & \\
\hline 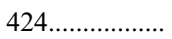 & 700096 & PMN J1131+0455 & 113158.0 & +045620.3 & 3.46 & 2000 Dec 15 & 6.37 & ACIS-S & 123678 & & \\
\hline $2126 \ldots \ldots \ldots \ldots \ldots$ & 700316 & $3 C 263$ & 114005.2 & +654759.7 & 1.15 & 2000 Oct 28 & 29.15 & ACIS-S & 235678 & & $\mathrm{Y}$ \\
\hline 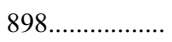 & 800074 & B1138-262 & 114046.1 & -263020.9 & 4.95 & 2000 Jun 06 & 23.50 & ACIS-S & 235678 & & $\mathrm{Y}$ \\
\hline $536 \ldots$ & 800044 & MS $1137.5+6625$ & 114047.1 & +6607 19.7 & 1.18 & 1999 Sep 30 & 114.61 & ACIS-I & 012367 & & $\mathrm{Y}$ \\
\hline $874 \ldots \ldots$ & 700179 & $1156+295$ & 115930.8 & +291428.4 & 1.66 & 2000 Jun 29 & 94.57 & ACIS-I & 012367 & & \\
\hline $2183 \ldots$ & 700373 & SDSS 1204-0021 & 120443.3 & -002119.6 & 2.13 & 2000 Dec 02 & 5.19 & ACIS-S & 235678 & & \\
\hline $2083 \ldots \ldots \ldots \ldots \ldots$ & 700273 & SDSS $120823+001027$ & 120824.2 & +001100.6 & 1.99 & 2001 Mar 18 & 4.52 & ACIS-S & 235678 & & \\
\hline $2103 \ldots \ldots \ldots \ldots \ldots$ & 700293 & LBQS $1205+1436$ & 120824.3 & +141952.6 & 2.56 & 2002 Mar 08 & 5.59 & ACIS-S & 235678 & & \\
\hline $2104 \ldots \ldots \ldots \ldots \ldots$ & 700294 & LBQS $1208+1535$ & 121124.4 & +151922.3 & 2.66 & 2002 Mar 08 & 4.01 & ACIS-S & 235678 & & \\
\hline $2106 \ldots \ldots \ldots \ldots \ldots$ & 700296 & LBQS $1216+1103$ & 121929.2 & +104729.0 & 2.14 & 2001 Mar 18 & 4.14 & ACIS-S & 235678 & & \\
\hline $1662 \ldots \ldots \ldots \ldots \ldots$ & 800130 & V $1221+4918$ & 122131.2 & +491507.5 & 1.43 & 2001 Aug 05 & 77.01 & ACIS-I & 01236 & & \\
\hline $2486 \ldots$ & 900080 & CHVC125 & 122737.4 & +752542.0 & 2.73 & 2001 Dec 24 & 20.54 & ACIS-S & 235678 & 15 & \\
\hline $938 \ldots \ldots$ & 900010 & AX J1227+4421 & 122745.0 & +442128.4 & 1.34 & 2000 Oct 04 & 4.75 & ACIS-I & 012367 & & \\
\hline $2484 \ldots \ldots \ldots \ldots \ldots$ & 900080 & CHVC125 & 122850.1 & +752423.8 & 2.71 & 2001 Oct 25 & 23.94 & ACIS-S & 235678 & 15 & \\
\hline $2253 \ldots \ldots \ldots \ldots \ldots$ & 900080 & CHVC125 & 122904.5 & +752543.5 & 2.69 & 2002 Jan 14 & 43.95 & ACIS-S & 235678 & 15 & \\
\hline $1712 \ldots$ & 790054 & $3 \mathrm{C} 273$ & 122906.3 & +02 0314.0 & 1.79 & 2000 Jun 14 & 12.04 & ACIS-S & 456789 & & $\mathrm{Y}$ \\
\hline $2107 \ldots \ldots \ldots \ldots \ldots$ & 700297 & LBQS $1231+1320$ & 123354.8 & +1303 35.4 & 2.57 & 2001 Aug 07 & 5.82 & ACIS-S & 235678 & & \\
\hline $2108 \ldots \ldots \ldots \ldots \ldots$ & 700298 & LBQS $1235+0857$ & 123753.8 & +084140.7 & 1.74 & 2001 Mar 18 & 5.33 & ACIS-S & 235678 & & \\
\hline $820 \ldots \ldots \ldots \ldots \ldots$ & 700125 & LBQS $1235+1807 B$ & 123822.0 & +1751 12.6 & 1.96 & 2000 Jan 21 & 1.24 & ACIS-S & 23678 & & \\
\hline $2109 \ldots \ldots \ldots \ldots \ldots$ & 700299 & LBQS 1239+0955 & 124134.8 & +093859.2 & 1.79 & 2001 Jul 25 & 5.06 & ACIS-S & 235678 & & \\
\hline $821 \ldots \ldots \ldots \ldots \ldots$ & 700126 & Q1246-0542 & 124915.6 & -055847.9 & 2.17 & $2000 \mathrm{Feb} 08$ & 5.38 & ACIS-S & 235678 & & \\
\hline $2084 \ldots \ldots \ldots \ldots \ldots$ & 700274 & PC $1247+3406$ & 124939.4 & +335010.0 & 1.23 & 2001 Mar 24 & 4.52 & ACIS-S & 235678 & & \\
\hline $1031 \ldots \ldots \ldots \ldots \ldots$ & 700389 & Mrk 231 & 125619.0 & +56 5224.6 & 1.25 & 2000 Oct 19 & 23.30 & ACIS-S & 235678 & & \\
\hline $2210 \ldots \ldots \ldots \ldots \ldots$ & 800149 & $3 \mathrm{C} 280$ & 125658.7 & +471948.2 & 1.15 & 2001 Aug 27 & 49.80 & ACIS-S & 235678 & & \\
\hline $325 \ldots \ldots \ldots \ldots \ldots$ & 800063 & S-Z CLUSTER & 131222.4 & +424142.8 & 1.37 & 1999 Dec 03 & 80.61 & ACIS-S & 23678 & & $\mathrm{Y}$ \\
\hline $2228 \ldots \ldots \ldots \ldots \ldots$ & 800167 & RX J1317.4+2911 & 131712.2 & +291018.3 & 1.04 & 2001 May 04 & 108.09 & ACIS-I & 01236 & & $\mathrm{Y}$ \\
\hline $2110 \ldots \ldots \ldots \ldots \ldots$ & 700300 & LBQS $1331-0108$ & 133428.8 & -012312.4 & 2.31 & 2001 Mar 18 & 4.96 & ACIS-S & 235678 & & \\
\hline $415 \ldots \ldots \ldots \ldots \ldots$ & 700087 & NGC 5273 & 134211.5 & +353734.9 & 0.96 & 2000 Sep 03 & 1.44 & ACIS-S & 235678 & & \\
\hline $2249 \ldots \ldots \ldots \ldots$ & 900076 & RX J13434+0001 & 134330.7 & +000204.7 & 1.90 & 2001 Jan 08 & 9.53 & ACIS-S & 235678 & 14 & \\
\hline $2250 \ldots \ldots \ldots \ldots$ & 900077 & F864X:052 & 134408.8 & -002801.6 & 2.02 & 2001 Jan 08 & 8.74 & ACIS-S & 235678 & & \\
\hline $2251 \ldots \ldots \ldots \ldots$ & 900078 & F864X:012 & 134426.6 & -000035.3 & 1.93 & 2001 Jan 08 & 9.54 & ACIS-S & 235678 & 14 & \\
\hline $809 \ldots \ldots \ldots \ldots \ldots$ & 700114 & MRK 273X & 134443.0 & +555416.4 & 1.09 & 2000 Apr 19 & 40.93 & ACIS-S & 012367 & & $\mathrm{Y}$ \\
\hline $507 \ldots \ldots \ldots \ldots \ldots$ & 800015 & RX J1347-114 & 134728.7 & -114624.2 & 4.88 & 2000 Apr 29 & 9.90 & ACIS-S & 235678 & & $\mathrm{Y}$ \\
\hline $2229 .$. & 800168 & RX J1350.0+6007 & 135055.1 & +600508.6 & 1.76 & 2001 Aug 29 & 54.86 & ACIS-I & 01236 & & \\
\hline $1588 \ldots \ldots \ldots \ldots$ & 700204 & $3 C 294$ & 140650.4 & +34 1120.0 & 1.21 & 2000 Oct 29 & 19.02 & ACIS-S & 235678 & & $\mathrm{Y}$ \\
\hline $578 \ldots \ldots \ldots \ldots \ldots$ & 890023 & $3 C 295$ & 141111.5 & +521301.6 & 1.34 & 1999 Aug 30 & 15.80 & ACIS-S & 235678 & 7 & $\mathrm{Y}$ \\
\hline $2254 \ldots \ldots$ & 900081 & $3 C 295$ & 141118.3 & +521210.3 & 1.34 & 2001 May 18 & 87.48 & ACIS-I & 012367 & 7 & \\
\hline $930 \ldots \ldots \ldots \ldots \ldots$ & 800106 & H1413+117 & 141543.9 & +113000.1 & 1.80 & 2000 Apr 19 & 24.09 & ACIS-S & 456789 & & $\mathrm{Y}$ \\
\hline $2024 \ldots \ldots \ldots \ldots \ldots$ & 600158 & RX J1416.4+2315 & 141628.0 & +231402.1 & 1.91 & 2001 Sep 05 & 14.47 & ACIS-S & 235678 & & \\
\hline $541 \ldots \ldots \ldots \ldots \ldots \ldots$ & 800049 & V1416+4446 & 141643.0 & +444828.5 & 1.24 & 1999 Dec 02 & 29.67 & ACIS-I & 01236 & & $\mathrm{Y}$ \\
\hline $1657 \ldots \ldots \ldots \ldots \ldots$ & 800125 & MACS J1423.8+2404 & 142336.2 & +240248.6 & 2.48 & 2001 Jun 01 & 18.23 & ACIS-I & 01236 & & \\
\hline $367 \ldots \ldots \ldots \ldots \ldots$ & 700039 & Q1422+231 & 142435.6 & +225543.7 & 2.69 & 2000 Jun 01 & 26.75 & ACIS-S & 123678 & & \\
\hline $907 \ldots \ldots \ldots \ldots \ldots \ldots$ & 800083 & QB 1429-008A,B & 143229.5 & -010558.4 & 3.54 & 2000 Mar 31 & 21.32 & ACIS-I & 01236 & & $\mathrm{Y}$ \\
\hline $2111 \ldots \ldots \ldots \ldots \ldots$ & 700301 & LBQS 1442-0011 & 144512.8 & -002421.1 & 3.69 & 2001 May 30 & 3.98 & ACIS-S & 235678 & & \\
\hline $2112 \ldots \ldots \ldots \ldots$ & 700302 & LBQS $1443+0141$ & 144545.7 & $+01: 29: 50.2$ & 3.47 & 2001 Mar 23 & 5.84 & ACIS-S & 235678 & & \\
\hline $939 \ldots \ldots \ldots \ldots \ldots$ & 900011 & AX J1510+0742 & 151022.2 & +074205.4 & 2.45 & 2000 Jun 01 & 4.54 & ACIS-I & 012367 & & \\
\hline $800 \ldots \ldots \ldots \ldots \ldots$ & 600103 & CB 58 & 151418.5 & +363611.7 & 1.35 & 2000 Jun 06 & 43.55 & ACIS-S & 235678 & & \\
\hline $800 \ldots \ldots \ldots \ldots \ldots$ & 600103 & CB 58 & 151418.5 & +363611.7 & 1.35 & $2000 \mathrm{Jul} 07$ & 43.55 & ACIS-S & 235678 & & \\
\hline $2085 \ldots \ldots \ldots \ldots \ldots$ & 700275 & SDSSJ153259-003944 & 153300.3 & -003906.5 & 6.25 & 2001 Mar 26 & 4.99 & ACIS-S & 235678 & & \\
\hline $869 \ldots \ldots \ldots \ldots \ldots$ & 700174 & ARP 220 & 153454.7 & +232952.5 & 4.28 & 2000 Jun 24 & 54.18 & ACIS-S & 235678 & & $\mathrm{Y}$ \\
\hline $822 \ldots \ldots \ldots \ldots \ldots$ & 700127 & SBSG $1542+541$ & 154358.3 & +535949.0 & 1.27 & 2000 Mar 22 & 4.05 & ACIS-S & 235678 & & \\
\hline $326 \ldots \ldots \ldots \ldots \ldots$ & 800064 & 3 C 324 & 154946.3 & +212519.3 & 4.30 & 2000 Jun 25 & 31.95 & ACIS-S & 235678 & & $\mathrm{Y}$ \\
\hline 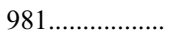 & 200088 & HD141569 & 154951.0 & -035538.3 & 9.59 & 2001 Jun 23 & 2.62 & ACIS-I & 012367 & & \\
\hline $2086 \ldots \ldots \ldots \ldots \ldots$ & 700276 & SDS S160501-011220 & 160459.2 & $-01 \quad 1244.5$ & 8.92 & 2001 Jun 24 & 4.54 & ACIS-S & 235678 & & \\
\hline $427 \ldots \ldots \ldots \ldots \ldots$ & 700099 & CLASS $1608+656$ & 160910.6 & +653226.2 & 2.68 & 2000 Jan 20 & 4.88 & ACIS-S & 01237 & & \\
\hline $2127 \ldots \ldots \ldots \ldots \ldots$ & 700317 & $3 C 330$ & 160939.8 & +655624.6 & 2.83 & 2001 Oct 16 & 40.23 & ACIS-S & 235678 & & \\
\hline $2184 \ldots \ldots \ldots \ldots \ldots$ & 700374 & SDSS $1621-0042$ & 162116.2 & -004325.0 & 7.26 & 2001 Sep 05 & 1.27 & ACIS-S & 235678 & & \\
\hline $546 \ldots \ldots \ldots \ldots \ldots$ & 800054 & MS $1621.5+2640$ & 162325.4 & +263612.4 & 3.59 & 2000 Apr 24 & 29.57 & ACIS-I & 01236 & & $\mathrm{Y}$ \\
\hline $615 \ldots \ldots \ldots \ldots \ldots$ & 200040 & VB 8 & 165534.0 & -082407.6 & 13.40 & 2000 Jul 10 & 8.54 & ACIS-S & 456789 & & $\mathrm{Y}$ \\
\hline $2221 \ldots \ldots \ldots \ldots$ & 800160 & 53W002 CLUSTER & 171416.5 & +501507.9 & 2.29 & 2001 Nov 01 & 35.97 & ACIS-S & 23678 & & \\
\hline 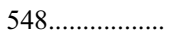 & 800056 & RX J1716.9+6708 & 171701.0 & +671144.1 & 3.71 & $2000 \mathrm{Feb} 27$ & 50.35 & ACIS-I & 01236 & & $\mathrm{Y}$ \\
\hline $841 \ldots \ldots \ldots \ldots \ldots$ & 700146 & $3 \mathrm{C} 371$ & 180652.3 & +695005.5 & 4.84 & 2000 Mar 21 & 9.43 & ACIS-S & 456789 & & $\mathrm{Y}$ \\
\hline
\end{tabular}


TABLE 1 - Continued

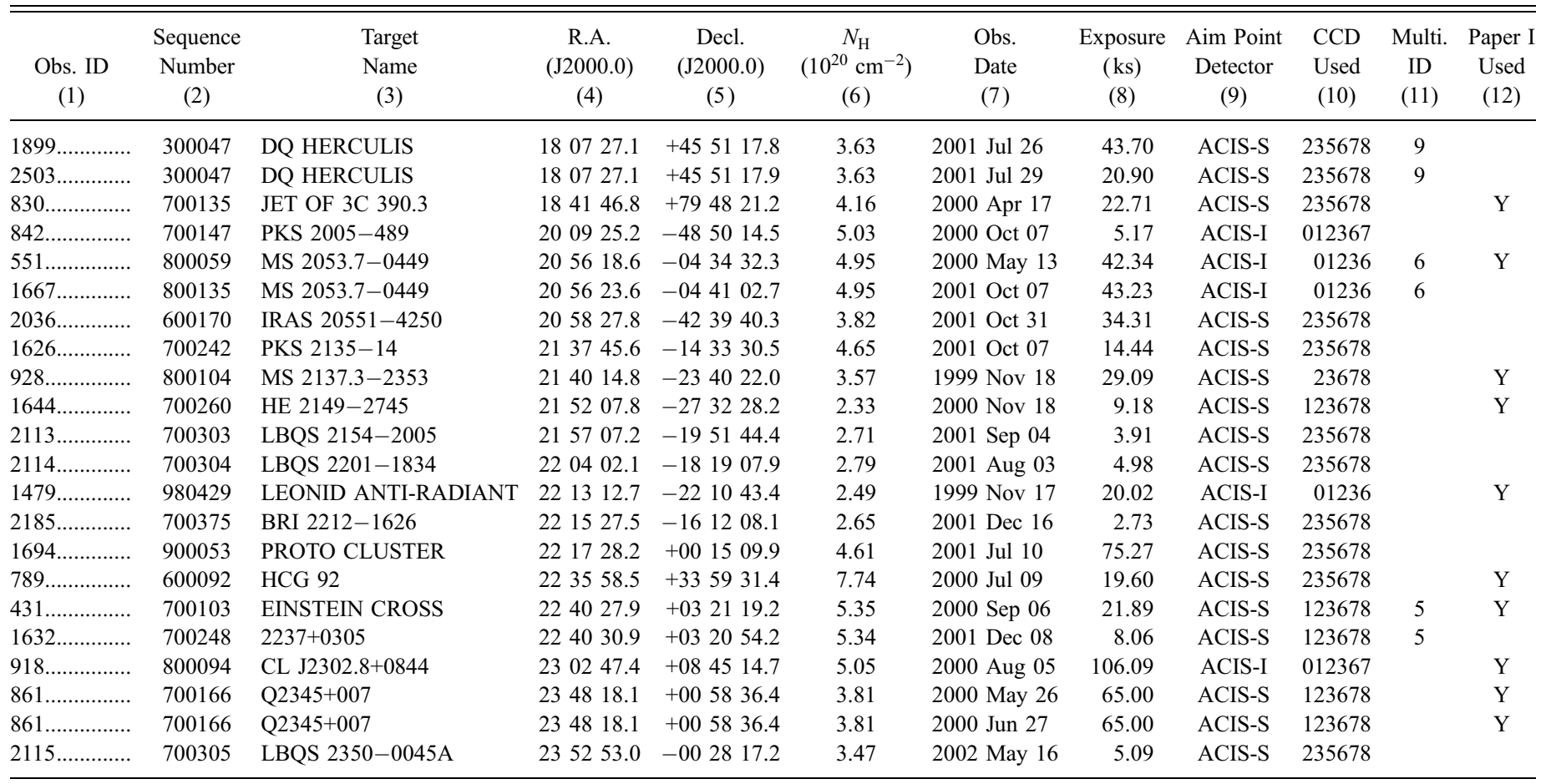

Notes.-Col. (1): Observational ID. Col. (2): Sequence number. Col. (3): Target name. Cols. (4) and (5): Right ascension in units of hours, minutes, and seconds, and declination in units of degrees, arcminutes, and arcseconds of the aim point in J2000.0. Col. (6): Galactic extinction in units of $10^{20} \mathrm{~cm}^{-2}$ from Stark et al. (1992). Col. (7): Date of observation. Col. (8): Net exposure time estimated after removing background flares within the aim pointed CCD chip. Col. (9): Aim point detector type. Col. (10): Used CCD chips (CCD ID 0-3 for ACIS-I and 4-9 for ACIS-S). Col. (11): Multiple observation ID indicates overlapping fields, labeled with the same ID. Col. (12): The 62 ChaMP fields included in Paper I are marked with "Y."

function (PSF) is highly asymmetrical and the background contribution to the counts in the source cell is non-negligible, are less accurate than the positions of on-axis sources (Paper I). To alleviate this problem, we applied a position refinement algorithm (P. Freeman 2003, private communication) that iteratively redetermines the position of the off-axis source until it converges on the best centroid (see Paper I for detail descriptions), to the $\mathrm{X}$-ray positions determined by wavdetect in CIAO 2.3. When
CIAO 3.0 or later versions are used, this process is not necessary, because the position refinement algorithm has been applied in wavdetect. ${ }^{10}$

The size and shape of the PSF for Chandra varies as a function of off-axis angle and radial direction. The wavdetect tool uses a Mexican Hat function, a reasonable function for mirrors/detectors

${ }^{10}$ See http://cxc.harvard.edu/ciao/releasenotes/ciao_3.0_release.html.

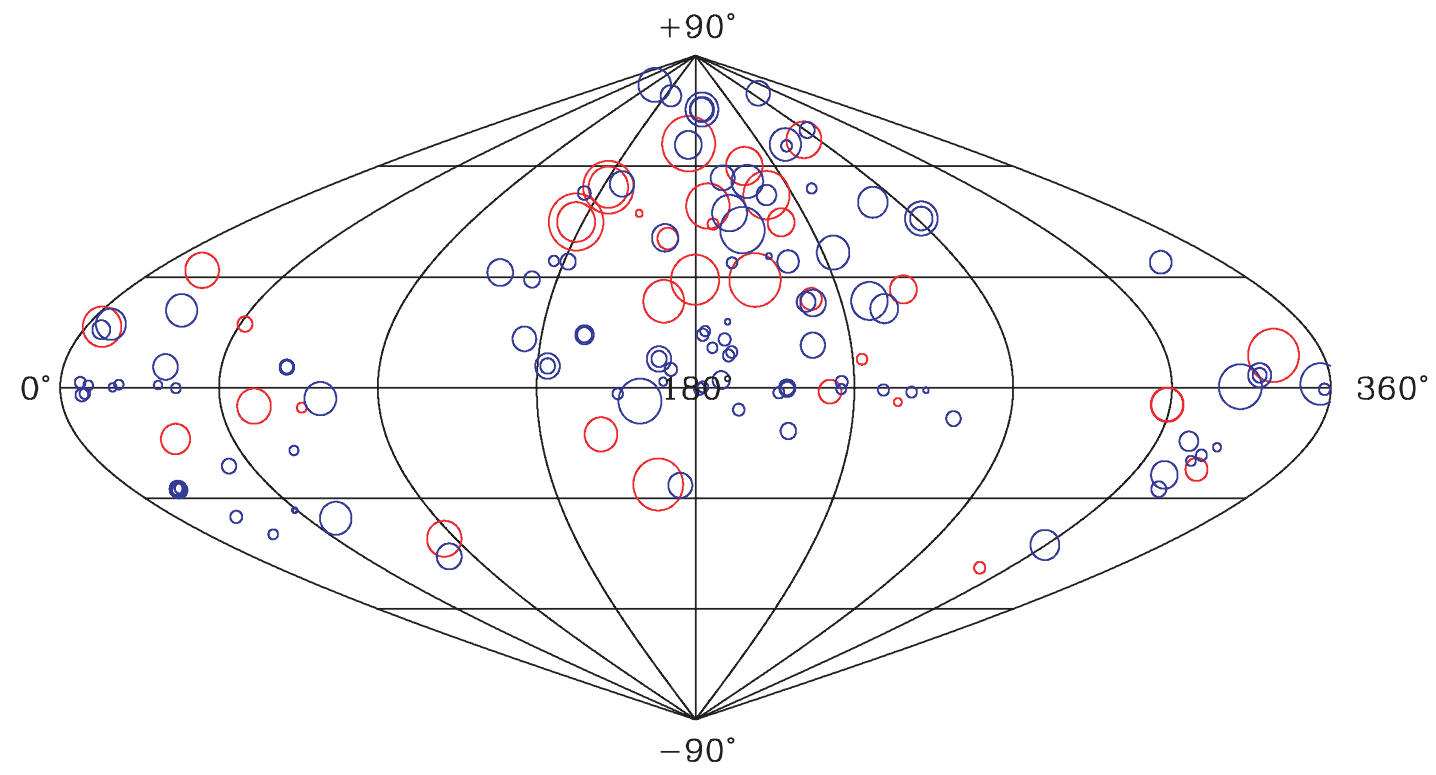

FIG. 1. - Location of 149 ChaMP fields in equatorial coordinates. Red circles represent ACIS-I at the aim point, and blue circles represent ACIS-S. The circle size crudely indicates the Chandra exposure time, ranging from 0.9 to $124 \mathrm{ks}$. The ChaMP fields are uniformly distributed over the celestial space except (by selection) the Galactic plane region. 

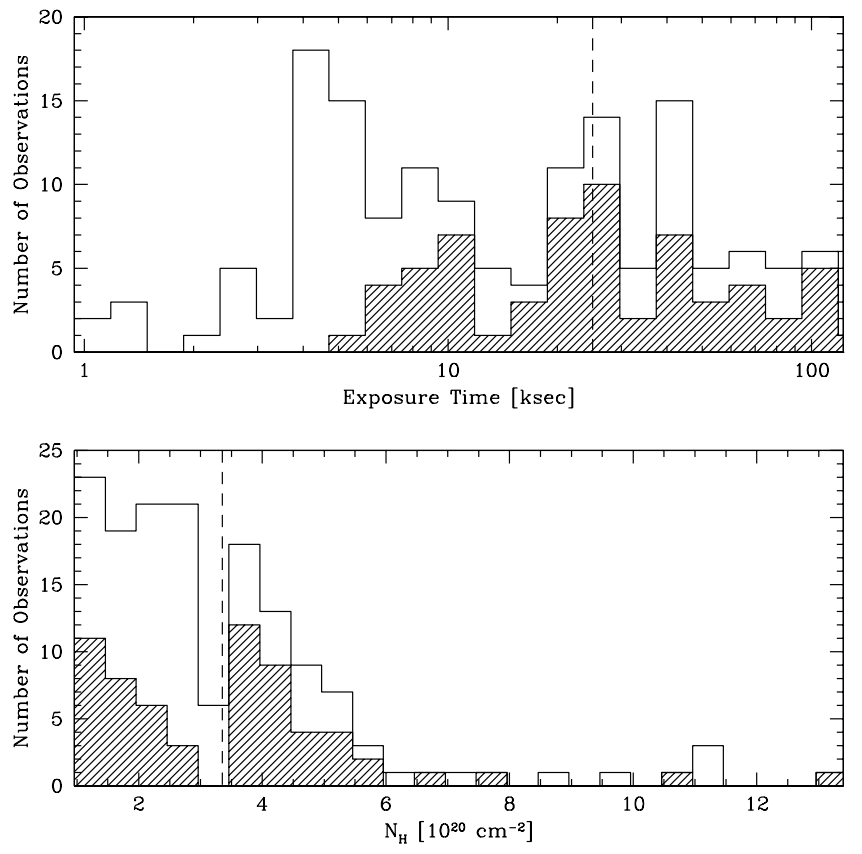

FIG. 2.-Number distribution of effective exposure times (top) and Galactic extinction $N_{\mathrm{H}}$ (bottom) of the 149 ChaMP observations. The dashed line indicates the mean of each number distribution. The mean exposure time of the ChaMP is $\sim 25 \mathrm{ks}$, and the mean Galactic extinction in the ChaMP is $N_{\mathrm{H}} \sim(3.4 \pm 2.2) \times$ $10^{20} \mathrm{~cm}^{-2}$. The ChaMP fields range from short to long exposure times, and their Galactic absorption is lower than the Galactic plane $\left(N_{\mathrm{H}} \sim 10^{22}-10^{23} \mathrm{~cm}^{-2}\right)$. The number distributions of the 62 ChaMP fields from Paper I are displayed as shaded histograms.

that are characterized by a quasi-Gaussian PSF, and one that detects sources successfully in most cases. However, the off-axis PSF is asymmetric and contains substructure in the core, causing wavdetect to detect sometimes a spurious pair of double sources. We note that the substructure of the off-axis PSF can be resolved by wavdetect because the size of the PSF becomes larger with increasing off-axis angle. This PSF effect can be corrected by a PSF deconvolution (Paper I). In Paper I, source pairs with small separations were inspected and three pairs of spurious doubles were found, bright (net counts $>$ few hundred counts) enough to deconvolve with their PSFs. In this study, to identify and correct this PSF effect, we generated a single PSF image at the median location of each overlapping pair of sources whose positional centers are very close together (see $\S 3.2 .2$ for quantitative definition of large overlapping sources) and a second image using a combination of two source PSFs. We then compared the observed source image with the modeled images. To generate the PSF images, we used a PSF ray trace tool ChaRT ${ }^{11}$ and a Chandra detector simulation tool MARX $^{12}$ assuming a monochromatic energy at $1.5 \mathrm{keV}$ and a source counts ratio corresponding to that of overlapping sources as determined by XPIPE.

We found 12 pairs of spurious double sources, and Figure 3 shows the observed sources image and the modeled images for a sample pair. First, we can see that the PSF shape at this location is asymmetric (middle) and the positional centers of the spurious double sources are located along the elongated PSF direction. The shape of the observed X-ray sources (left) is similar to the single PSF (middle) rather than that of the double-source PSFs (right). Therefore, we conclude that wavdetect has incorrectly detected a single source as a double source due to the asymmetric

\footnotetext{
11 See http://asc.harvard.edu/chart/threads/index.html.
}

12 See http://space.mit.edu/CXC/MARX/.
TABLE 2

Definition of Energy Bands and X-Ray Colors

\begin{tabular}{|c|c|}
\hline Band & Definition \\
\hline Broad (B)... & $0.3-8 \mathrm{keV}$ \\
\hline Soft $(\mathrm{S}) \ldots \ldots \ldots$ & $0.3-2.5 \mathrm{keV}$ \\
\hline 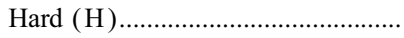 & $2.5-8 \mathrm{keV}$ \\
\hline Soft1 $\left(\mathrm{S}_{1}\right)$ & $0.3-0.9 \mathrm{keV}$ \\
\hline Soft2 $\left(\mathrm{S}_{2}\right)$ & $0.9-2.5 \mathrm{keV}$ \\
\hline Conventional broad $(\mathrm{Bc}) \ldots \ldots \ldots \ldots \ldots . . . . .$. & $0.5-8 \mathrm{keV}$ \\
\hline Conventional soft $(\mathrm{Sc}) \ldots \ldots \ldots \ldots \ldots \ldots \ldots$ & $0.5-2 \mathrm{keV}$ \\
\hline Conventional hard $(\mathrm{Hc}) \ldots \ldots \ldots \ldots \ldots \ldots . . . . . . .$. & $2-8 \mathrm{keV}$ \\
\hline 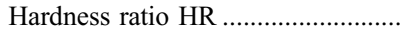 & $(\mathrm{Hc}-\mathrm{Sc}) /(\mathrm{Hc}+\mathrm{Sc})$ \\
\hline 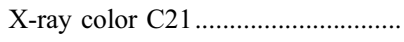 & $-\log \left(\mathrm{S}_{2}\right)+\log \left(\mathrm{S}_{1}\right)=\log \left(\mathrm{S}_{1} / \mathrm{S}_{2}\right)$ \\
\hline 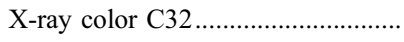 & $-\log (\mathrm{H})+\log \left(\mathrm{S}_{2}\right)=\log \left(\mathrm{S}_{2} / \mathrm{H}\right)$ \\
\hline
\end{tabular}

substructure in the PSF. All double sources found to be spurious are too faint (net counts $<$ few hundred counts) to deconvolve with their PSFs. Therefore, we assigned the median position of the spurious double sources as the new position of the single source and half of the distance between double sources was quadratically summed to their positional uncertainties (see $\S 4.2 .1$ for the positional uncertainty of the ChaMP X-ray point sources). The source counts were then extracted at the new source position (see $\S 3.2$ for the source counts extraction).

\subsection{Source Properties}

After detecting X-ray sources with wavdetect, we extracted their X-ray properties by applying aperture photometry. Since wavdetect sometimes underestimates the net counts for faint sources (see $\S 4.3$ ), we do not use the wavdetect-determined $\mathrm{X}$-ray photometry. Instead, we apply an aperture photometry source extraction tool xapphot, developed for a general purpose and applicable to both Chandra and XMM-Newton data (E. Kim et al. 2007 , in preparation). We note that XPIPE detects the source positions only in the B band with wavdetect and applies the same position and size for the source extraction regions in every energy band. The reliability of the ChaMP source properties using XPIPE will be discussed in $\S 4.3$.

\subsubsection{Source Count Extraction Regions}

With the X-ray source position determined by wavdetect, we extract source counts from a circle with a $95 \%$ encircled energy radius, determined at $1.5 \mathrm{keV}$ from the PSF table. ${ }^{13}$ A minimum radius of $3^{\prime \prime}$ and maximum of $40^{\prime \prime}$ are chosen to avoid small number statistics in the source counts and severe fluctuations in the background sky. The source radii in this study are slightly smaller than those in Paper I, which used an older version of the PSF tables. In the top panel of Figure 4, the source radius for Paper I (dotted line) and that for this study (solid line) are displayed as a function of off-axis angle. The difference between source sizes is plotted as a dashed line, indicating a significant difference at large off-axis angle. The source size is reduced by a maximum of $\sim 18^{\prime \prime}$ at off-axis angle of $\sim 13^{\prime}$ and unchanged in the axis region of $\lesssim 3^{\prime}$ compared to the old source size. In the bottom panel of Figure 4 , the difference between source counts in this and previous studies are displayed. The reduced source radii yield average net counts lower by $2 \% \pm 7 \%$ in this catalog compared with Paper I.

The size of the background extraction annulus is a free parameter generally chosen within the range 2-5 times the source radius, depending on local and global background fluctuations.

13 See http://cxc.harvard.edu/cal/Hrma/psf/index.html. 

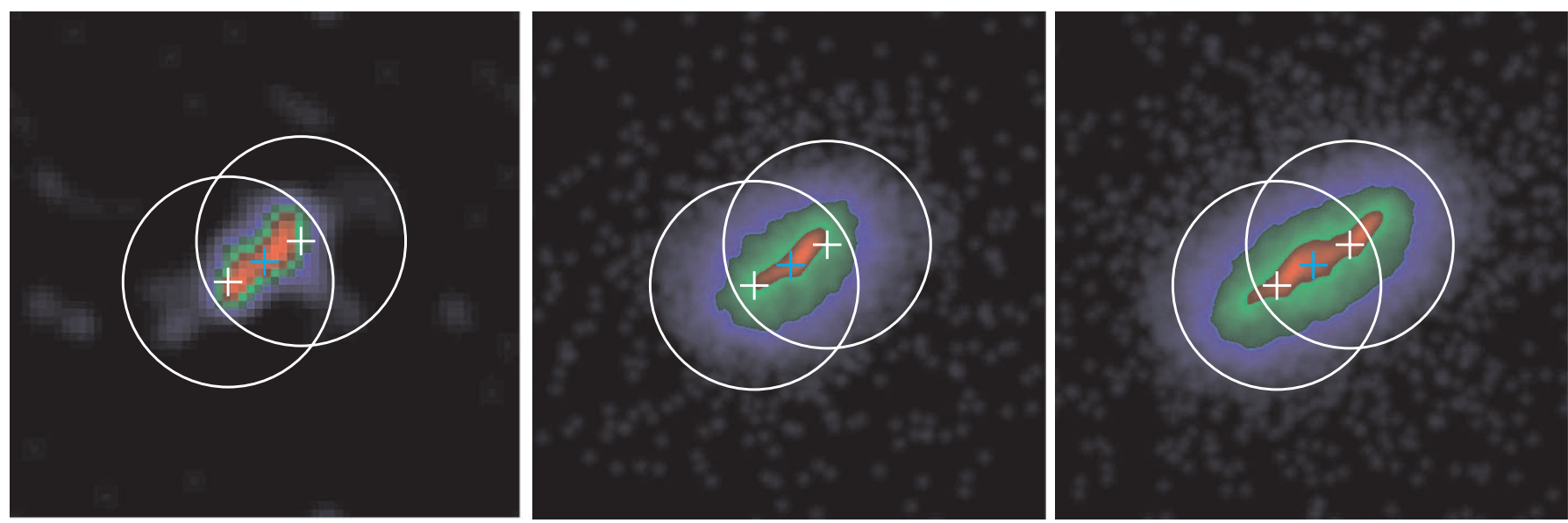

FIG. 3.- - Large overlapping double X-ray sources detected by wavdetect (left), the PSF image at their median location (middle), and the combined image of two PSFs at each X-ray source position (right). The white crosses and circles mark the positions and sizes of the double sources. The cyan crosses indicate the median location of the double sources. Circle size corresponds to the source extraction region used in xapphot. The shape of the detected overlapping X-ray sources (left) are similar to the shape of the single PSF (middle), rather than double PSFs (right). We conclude that the overlapping X-ray sources are spurious doubles due to the elongated PSF shape and substructures in the PSF. The PSF images are generated by the ChaRT tool in the CIAO package and MARX package. The size of all images is $\sim 30^{\prime \prime} \times 30^{\prime \prime}$, and all images are aligned with the world coordinate system.

However, for point sources inside an extended source, the size of the background extraction annulus was set to 1-2 times the source radius because in this case local variations in the background are much more important than global variations. The ChaMP X-ray extended sources are identified by wavdetect with a large wavelet and source properties extracted via fitting with a Gaussian profile and a $\beta$ model. The ChaMP extended source catalog used in the ChaMP point source photometry is provided in a separate paper (Barkhouse et al. 2006).
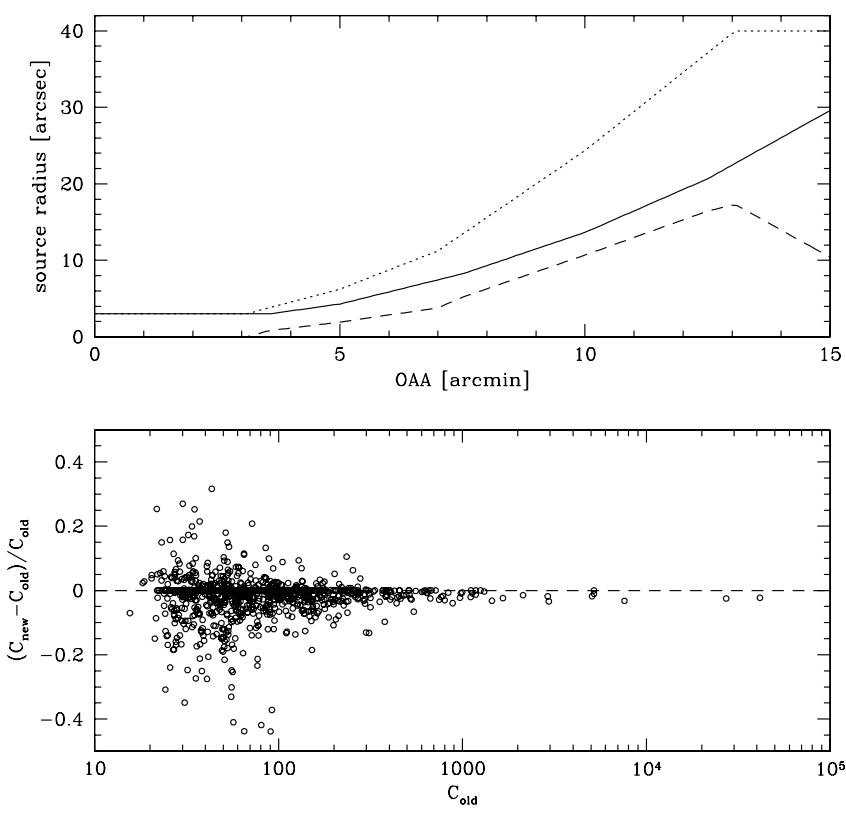

FIG. 4.- (Top) Source extraction radius as a function of off-axis angle. The dotted line represents the source radius from the old version of the CIAO CALDB used in the previous ChaMP catalog (Paper I). The solid line represents the source radius from the latest version of the CIAO CALDB used in this catalog. The source size is limited to a minimum radius of $3^{\prime \prime}$ and a maximum of $40^{\prime \prime}$. The difference between the two radii is plotted as a dashed line. (Bottom) The difference between the source counts in this and the previous catalog as a function of the previous counts. On average the reduced source radius yields source counts lower by $\sim 2 \% \pm 7 \%$ in this catalog. The dashed line indicates the zero difference level.

\subsubsection{Net Counts}

The net counts $N$ of a source in a given energy band are determined by subtracting the normalized background counts from the source counts in the source region as follows:

$$
N=N_{S}-N_{B} / \mathrm{AR}
$$

where $N_{S}$ and $N_{B}$ are the total counts in the source and background regions, respectively. The normalization factor $\mathrm{AR}$ in equation (1) is given by

$$
\mathrm{AR}=\frac{\left\langle E_{B}\right\rangle A_{B}}{\left\langle E_{S}\right\rangle A_{S}},
$$

where $\left\langle E_{S}\right\rangle$ and $\left\langle E_{B}\right\rangle$ are the mean exposure times for the source and background regions, respectively, and $A_{S}$ and $A_{B}$ are the geometric areas of the two regions. To avoid contamination in the background region, we exclude other point and extended source regions within the background region. The net counts errors are derived following Gehrels (1986).

There is a significant probability that two or more sources will overlap with each other, especially at large off-axis angle. Note that the PSF size increases exponentially with increasing off-axis angle. For overlapping sources, simple aperture photometry overestimates the source counts. While simultaneous fitting of multiple PSFs may be a good way to deconvolve overlapping sources, this process requires sufficient counts (a few hundred), which is unusual for typical X-ray observations. Thus, to determine the net counts for overlapping sources, we apply two independent correction methods as in Paper I, a small overlap correction and a large overlap correction, depending on the amount of overlapping area involved.

In the left side of Figure 5, we display a schematic diagram of a small overlapping source, in which the distance between them $D_{12}$ is greater than the radius of each source but less than the sum of their radii:

$$
0.5<\frac{D_{12}}{D_{\mathrm{PSF}}}<1
$$



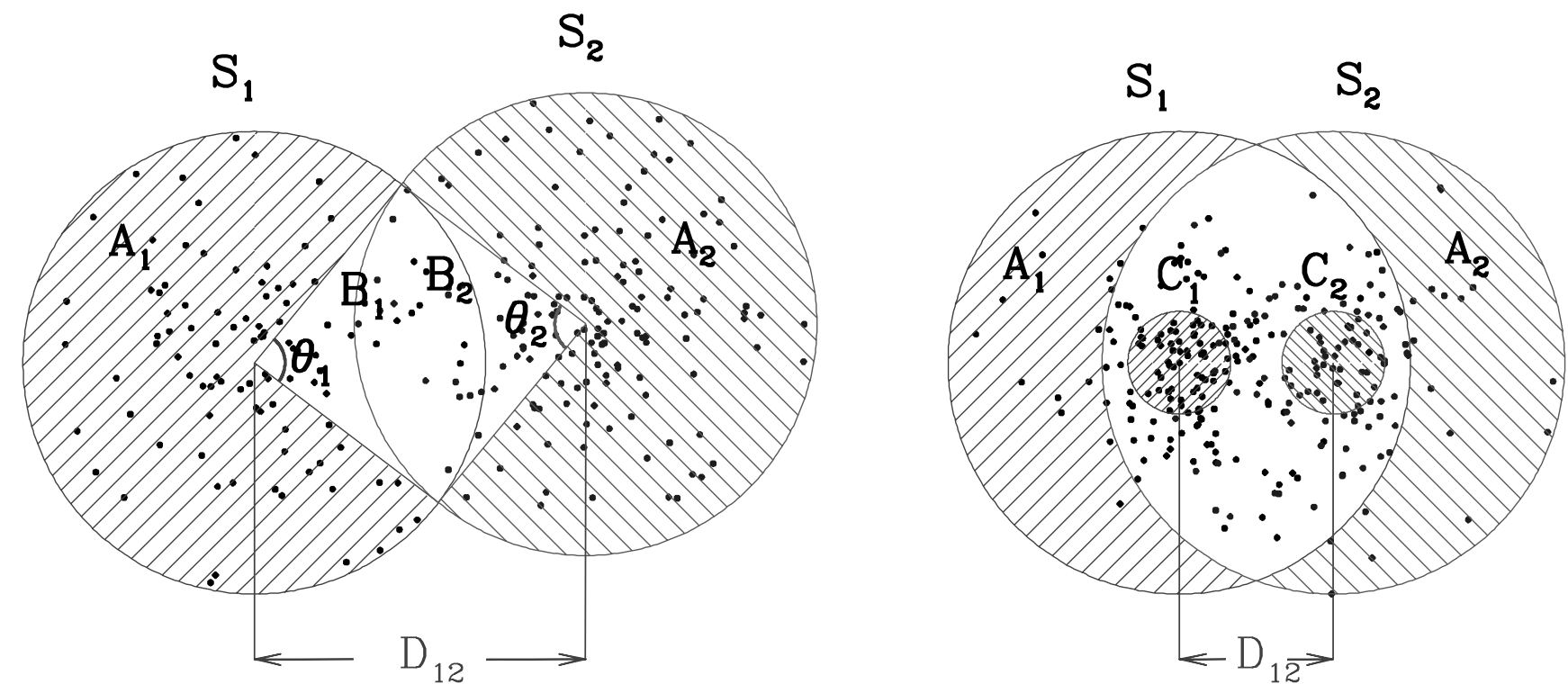

FIG. 5.-Schematic diagrams of the two types of overlapping X-ray source pairs, small (left) and large (right). $S_{1}$ and $S_{2}$ represent the source 1 and source 2, respectively. $A_{1}$ and $A_{2}$ represent the independent area of each source. $\theta_{1}$ and $\theta_{2}$ represent the angles covered by sectors $B_{1}$ and $B_{2}$ of overlapping regions of two sources, respectively. $C_{1}$ and $C_{2}$ represent the core regions of large overlapping sources, respectively. $D_{12}$ represents the distance between $S_{1}$ and $S_{2}$. The points are simulated X-ray events assuming a $\beta$ model for the event distribution. For the small overlapping case, only the photons in regions $A_{1}$ and $A_{2}$ are used to determine the correction, while the photons in regions $A^{\prime} s$ and $C^{\prime} s$ are used in the large overlapping case. The detailed correction method of xapphot is described in the text. [See the electronic edition of the Supplement for a color version of this figure.]

where $D_{\mathrm{PSF}}$ is the $95 \%$ encircled energy diameter of the PSF. A small overlap is the most common overlap among X-ray sources due to their relatively low density in the ChaMP fields. The corrected net counts $N_{1}$ and $N_{2}$ for the overlapping sources S1 and $\mathrm{S} 2$ are estimated as follows:

$$
\begin{aligned}
& N_{1}=2 \pi N_{1, A_{1}} /\left(2 \pi-\theta_{1}\right), \\
& N_{2}=2 \pi N_{2, A_{2}} /\left(2 \pi-\theta_{2}\right),
\end{aligned}
$$

where $N_{1, A_{1}}$ and $N_{2, A_{2}}$ are the net counts of S1 in area $A_{1}$ and the net counts of S2 in area $A_{2}$, respectively, and $\theta_{1}$ and $\theta_{2}$ are the angles in units of radians covered by sectors $B_{1}$ and $B_{2}$ of the overlapping sources, respectively. We assumed a radially symmetric event distribution for both X-ray sources.

The right side of Figure 5 displays an example of large overlapping sources, where the distance $D_{12}$ is less than the radius of each source. In this case, the center of each source is located within the source region of the overlapping partner, such that the above algorithm is not applicable. To correct this large overlapping case, first, we defined the core radius $R_{c}$ of each source as follows:

$$
R_{c} \equiv \max \left(\frac{D_{12}}{3}, 2 \text { pixels }\right)
$$

where $D_{12}$ is the distance between two overlapping sources. The radius $R_{c}$ has a minimum of 2 pixels to allow a statistically robust estimation of the counts within the core radius. The corrected net counts of the large overlapping sources $N_{1}$ and $N_{2}$ are estimated as follows:

$$
\begin{gathered}
N_{1}=N_{t}\left(2 N_{1, A_{1}}+N_{1, C_{1}}\right) / N_{0}, \\
N_{2}=N_{t}\left(2 N_{2, A_{2}}+N_{2, C_{2}}\right) / N_{0}, \\
N_{0}=\left(2 N_{1, A_{1}}+N_{1, C_{1}}\right)+\left(2 N_{2, A_{2}}+N_{2, C_{2}}\right),
\end{gathered}
$$

where $N_{t}$ is the sum of net counts of S1 and S2 (i.e., net counts in a union area of two source regions). The quantities $N_{1, A_{1}}$ and $N_{2, A_{2}}$ are the net counts of $\mathrm{S} 1$ in region $A_{1}$ and the net counts of $\mathrm{S} 2$ in region $A_{2}$, respectively; $N_{1, C_{1}}$ and $N_{2, C_{2}}$ are the net counts of $\mathrm{S} 1$ in core region $C_{1}$ and the net counts of $\mathrm{S} 2$ in core region $C_{2}$, respectively. The radii of core regions $C_{1}$ and $C_{2}$ are calculated with equation (6) and have the same size. Since the core region of each source is contaminated by photons from the overlapping source, the photons in unperturbed regions $\left(A_{1}\right.$ and $\left.A_{2}\right)$ are weighted higher than those in core regions $\left(C_{1}\right.$ and $\left.C_{2}\right)$ by a factor of 2. Excluding spurious sources, small and large overlap corrections were applied to $2.5 \%$ and $0.6 \%$ of the ChaMP sources, respectively. We note that xapphot does not include the correction procedure for a source overlapping largely with more than one source. However, only one such case is included in the ChaMP X-ray point source catalog: CXOMP J111816.9+074558, CXOMP J111816.8+074600, and CXOMP J111816.8+074557 largely overlap each other, and they are the target of the observation (OBSID 363 , gravitationally lensed quasar) having a pile-up flag (flag 37 , see Table 4 in $\S 3.2 .5$ ).

\subsubsection{Hardness Ratio and Colors}

The X-ray point source properties are extracted in the five ChaMP specified energy bands and in the three commonly used energy bands. The used energy bands and definitions of hardness ratio (HR) and X-ray colors (C21 and C32) are listed in Table 2, and their scientific rationale was described in Paper I. The HR and X-ray colors can be calculated from the source net counts in two different energy bands according to their definitions (hereafter classical method). However, for the faint sources, the HR, C21, and $\mathrm{C} 32$ and their error propagations from the classical method are often unreliable or unrealistic because of negative/undetectable net counts in one band or a non-Gaussian nature. Therefore, we calculated the HR, C21, and C32 with a Bayesian approach that models the detected counts as a Poisson distribution and that gives 

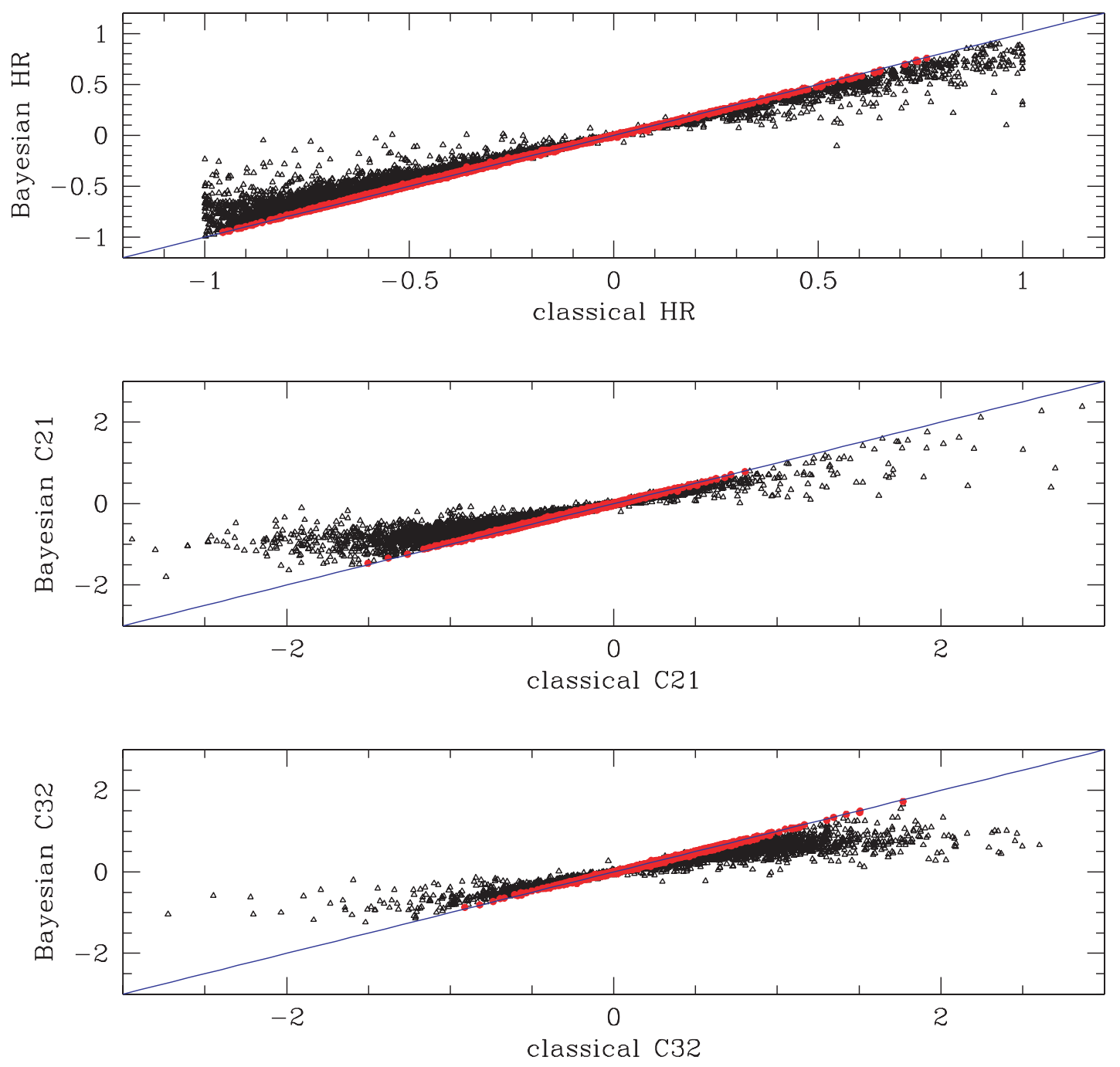

FIG. 6.-Comparison of a classical with a Bayesian method for HR (top), C21 (middle), and C32 (bottom). The sources with net counts $\geq 0$ (net counts $>0$ ) for HR (X-ray colors) in both energy bands are plotted. The black open triangles represent the sources with $\mathrm{S} / \mathrm{N}<2$ in at least one energy band. The red closed circles represent the sources with $\mathrm{S} / \mathrm{N}>2$ in two energy bands. The blue line represents the line of equality for the two methods and is shown for illustrative comparison. For bright sources $(\mathrm{S} / \mathrm{N}>2)$, the HR and X-ray colors from both methods agree well; however, for faint sources $(\mathrm{S} / \mathrm{N}<2)$, they do not agree, because the classical method, using Gaussian statistics, fails to describe the nature of faint sources.

reliable HR and X-ray colors for both low- and high-count sources (van Dyk et al. 2004; Park et al. 2006).

To derive the HR and X-ray colors with the Bayesian approach, we used the BEHR ${ }^{14}$ program (ver. 07-27-2006; Park et al. 2006) with the required inputs: source counts, background counts, and ratio of background area to source area in both energy bands. We assumed a noninformative, flat, prior distribution on the linear scale (soft $\mathrm{idx}=$ hard $\mathrm{idx}=1$ ). We note that the energydependent vignetting in the soft and hard counts is not corrected for deriving the HR. The BEHR program calculates the solution with two different methods: a Gibbs sampler (Monte Carlo integration) and a Gaussian quadrature (numerical integration). The Gibbs sampler is efficient but less accurate than the Gaussian quadrature for faint sources; however, the Gaussian quadrature becomes less efficient with increasing source counts. Therefore, we used the Gibbs sampler for bright sources (net counts $>15$ in two energy bands) and Gaussian quadrature for faint sources (net counts $<15$ in at least one energy band), respectively. The default values were used for the remaining optional inputs. The

\footnotetext{
14 See http://hea-www.harvard.edu/AstroStat/BEHR/.
}

BEHR program calculates the mode, mean, and median of the posterior probability distribution. The mean of the distribution is a robust estimator for the $\mathrm{HR}$, while the mode estimates the X-ray colors (Park et al. 2006). In Figure 6, we compare the classical method with the Bayesian approach for HR and X-ray colors. For bright sources $(\mathrm{S} / \mathrm{N}>2)$, the HR and X-ray colors from both methods agree well; however, for faint sources $(\mathrm{S} / \mathrm{N}<2)$, they do not agree, because the classical method using the Gaussian statistics fails to describe the nature of faint sources.

\subsubsection{Source Flux}

In general, the Chandra X-ray source flux is determined as follows:

$$
\text { flux }=\text { count rate } \times \mathrm{ECF} \text {, }
$$

where ECF is the energy conversion factor, which converts source count rate to source flux in units of ergs $\mathrm{cm}^{-2}$ count $^{-1}$. The ECF varies with observation date and CCD pixel position because of the temporal and spatial variations of the ACIS CCD quantum 

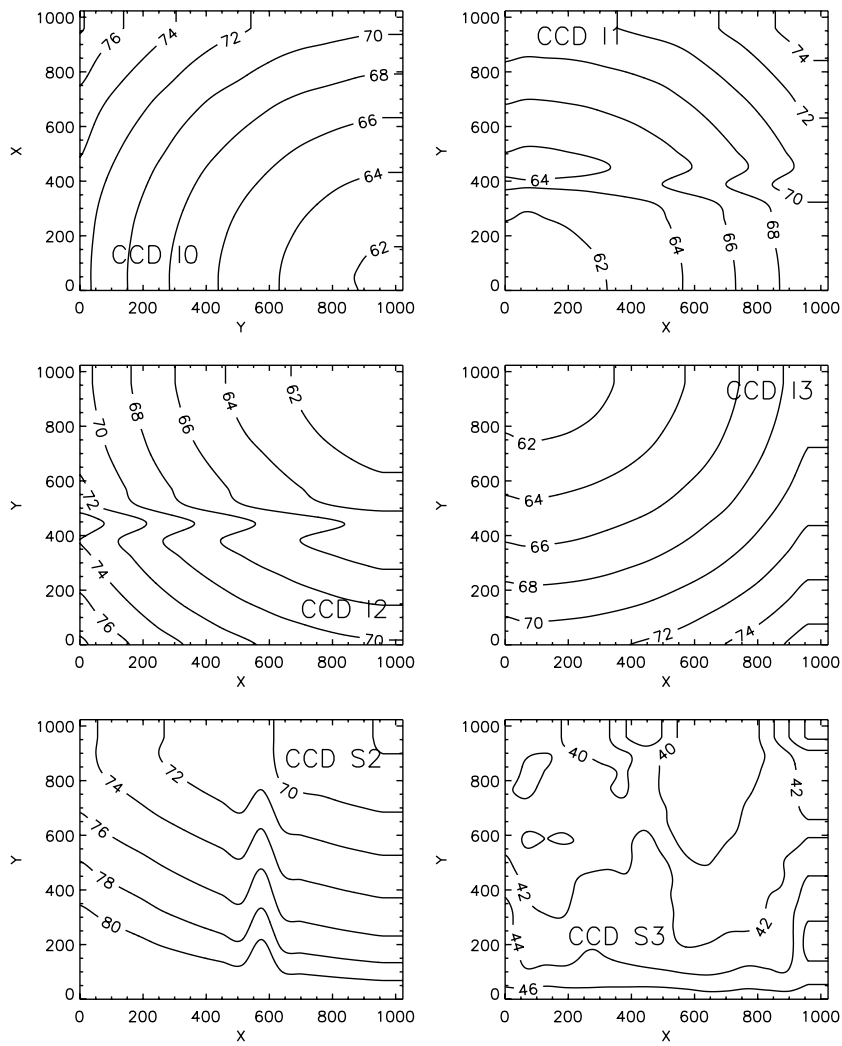

FIG. 7.-Contour maps of the energy conversion factors (ECF) in ACIS-I observations including ACIS-S S2 and S3 CCD chips. Figures are shown in chip coordinates rotated by $90^{\circ}$ (CCD I1 and I3) and $270^{\circ}$ (CCD I0 and I2) relative to CCD S3. The energy band is $0.3-2.5 \mathrm{keV}$, and a photon index of $\Gamma_{\mathrm{ph}}=1.7$ was assumed. The ECFs are calculated at $16 \times 16$ grid points with a grid size of 32 pixels and smoothed with a cubic kernel. The ECF is in units of ergs $\mathrm{cm}^{-2}$ count $^{-1}$.

efficiency ${ }^{15}$ and the vignetting effect. The temporal QE variation of the ECF can be corrected by generating ECFs per observation and per CCD chip. To investigate the spatial variation of the ECF, we generated the $0.3-2.5 \mathrm{keV}$ ECF map of an ACIS-I observation including ACIS-S S2 and S3 CCD chips. Using the redistribution matrix function (RMF) and ancillary response function (ARF) files and assuming a photon index of $\Gamma_{\mathrm{ph}}=1.7$ and Galactic absorption $N_{\mathrm{H}}$ (Stark et al. 1992) for a given observation, we derive ECFs with Sherpa ${ }^{16}$ in $16 \times 16$ grid points with grid size of 32 pixels in each CCD chip (here after $\mathrm{ECF}_{\text {grid }}$ ). In Figure 7, we display the $\mathrm{ECF}_{\text {grid }}$ contour maps smoothed with a cubic kernel, and the left panels of Figure 8 show $\mathrm{ECF}_{\text {grid }}$ as a function of off-axis angle in each CCD chip. The ECF spatially varies by up to $\sim 25 \%$.

To quantitatively see the spatial variation of the $\mathrm{QE}$, we display the ratios of $\mathrm{ECF}_{\text {grid }}$ over the $V_{\text {cor }} \mathrm{ECF}_{\text {single }}$ as a function of the offaxis angle in the right panels of Figure 8, where $\mathrm{ECF}_{\text {single }}$ is the ECF calculated at a single position (the aim point position for ACIS-I CCDs and at the maximum exposure positions for $\mathrm{S} 2$ and $\mathrm{S} 3$ chips) and $V_{\text {cor }}$ is the vignetting correction factor, which is estimated from the exposure map at each grid position. The vignettingcorrected $\mathrm{ECF}_{\text {single }}$ agrees well with $\mathrm{ECF}_{\text {grid }}$ with the exception of points that are estimated from the CCD edge and bad pixels/ columns (blue squares). The spatial variation of the $\mathrm{QE}$ is shown at large off-axis angle (red triangles); however, the deviation is less than $5 \%$. Therefore, in this study, we ignore the spatial varia-

\footnotetext{
${ }^{15}$ See http://asc.harvard.edu/cal/Acis/Cal_prods/qeDeg for the low-energy QE degradation.

${ }_{16}$ See http://asc.harvard.edu/sherpa/threads/index.html.
}

tion of the QE and correct the vignetting effect to determine the source flux as follows:

$$
\text { flux }=\text { count rate } \times \mathrm{ECF}_{\text {single }} \times V_{\text {cor }} .
$$

In Table 3, $\mathrm{ECF}_{\text {single }}$ are listed per observation (OBSID) and per CCD chip and calculated at the aim point for I0-I3 of ACIS-I observation and S3 of ACIS-S observation. For the remaining chips, $\mathrm{ECF}_{\text {single }}$ is calculated at the maximum exposure position. For general usage, we calculated $\mathrm{ECF}_{\text {single }}$ assuming various photon indices, $\Gamma_{\mathrm{ph}}=1.2,1.4$, and 1.7 and Galactic absorption $N_{\mathrm{H}}$ from Stark et al. (1992) for that observation. We provide the effective exposure time of each X-ray source corrected for the vignetting effect at the source position (see Tables 5 and 6 in $\S 5.1$.

\subsubsection{Source Flags}

All X-ray sources in the ChaMP catalog have been visually inspected to flag those sources with various special issues, as listed in Table 4. Flags $11-51,53$, and 54 were determined only by visual examination. The spurious double sources due to PSF effects (flags 15 and 38) are described in detail in $\S 3.1$. Since 35 of the 149 ChaMP fields partly overlap on the sky, as seen in the eleventh column of Table 1, 453 sources were likely observed more than once (flag 52) in these overlapping fields. We identified these 453 source candidates by their positions, matching sources in multiply observed fields within a $95 \%$ confidence level positional uncertainty (see eq. [12] in $\S 4.2 .1$ ). False sources having flags from 11 to 21 and extended X-ray sources (flag 51) including the X-ray jets (flag 54) are not listed in the ChaMP X-ray point source catalogs.

To remove bad pixels/columns, we used the bad pixel file. Additional hot pixels and bad columns were identified by visually inspecting each CCD image and an event histogram as a function of chip $x$-coordinate (see $\S 3.1$ in Paper I for details). The bad pixels are then included in generating an exposure map, which is in turn used to calculate the count rate and flux. Although the source flux may be slightly underestimated when a bad pixel sits at a source location, the effect of a single bad pixel is considerably mitigated by the aspect dither (following a Lissajous pattern over $16 \times 16 \operatorname{arcsec}^{2}$ ). Therefore, we flagged sources within which a bad pixel/column exists as flag 31 following visual inspection.

When the source is located at the edge of the CCD chip, where the minimum exposure value in the source region is less than $10 \%$ of the maximum exposure value, flag 61 is assigned. The edge flag 61 and overlapping flags from 62 to 68 are automatically flagged by xapphot. The overlapping flags correspond to flags 32-35 in Paper I, with more detailed classes included here: the overlapping class is subclassified as either small or large overlaps (see $\S 3.2 .2$ for the definition of small and large overlap).

\section{ChaMP X-RAY POINT SOURCE SIMULATIONS}

\subsection{Simulation Procedure}

To investigate source reliability and sensitivity, and to establish the empirical equations for positional uncertainty on X-ray sources in the ChaMP fields, we have performed extensive simulations. The technique was based on that of Kim \& Fabbiano (2003) and consists of three parts: (1) generating artificial X-ray sources with MARX,${ }^{17}(2)$ adding them to the observed image, and (3) detecting these artificial sources with wavdetect and extracting source properties with the xapphot. We have used every

${ }^{17}$ See http://space.mit.edu/CXC/MARX/and MARX 4.0 Technical Manual. 

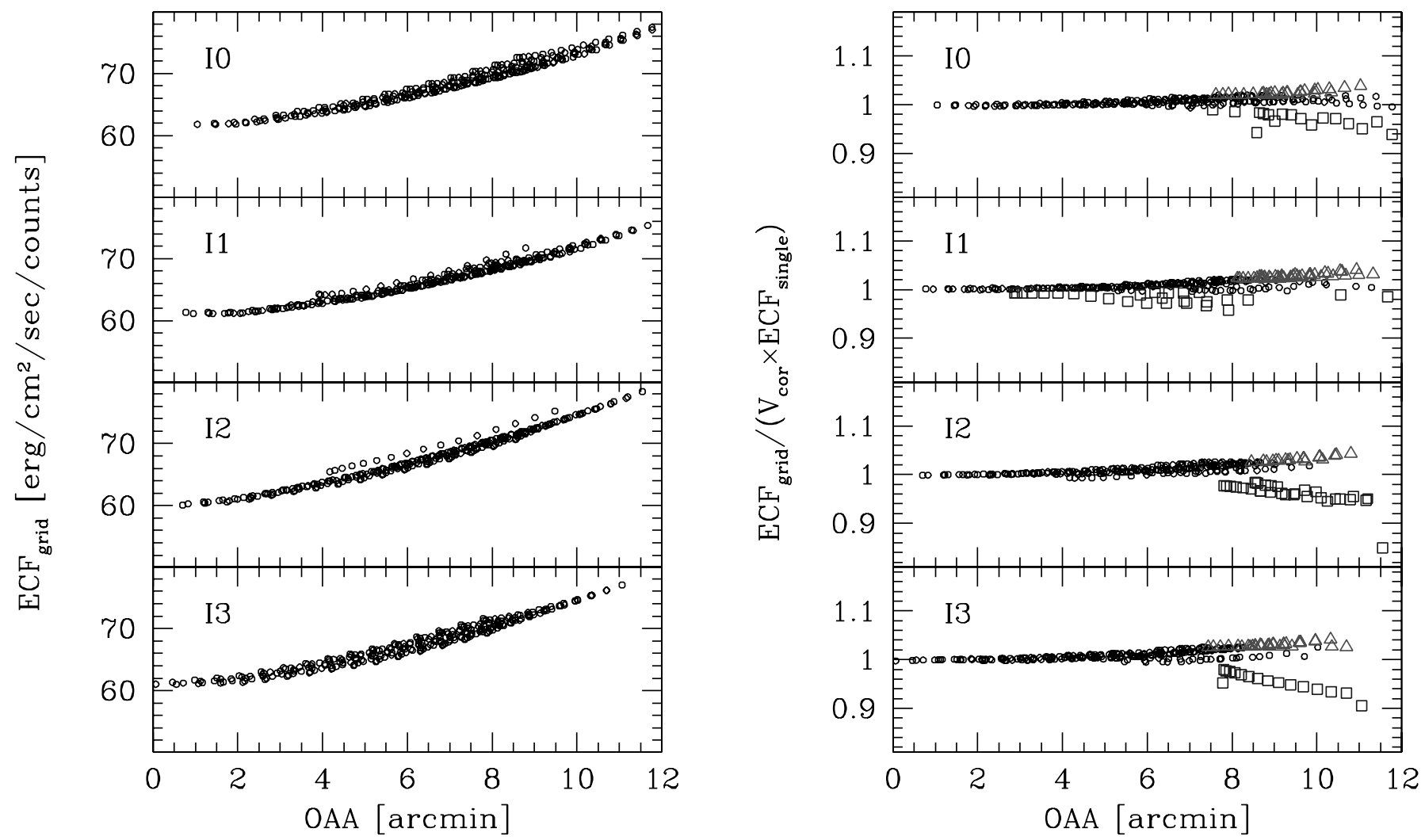

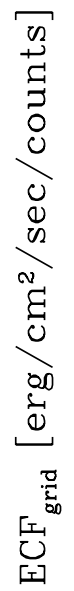
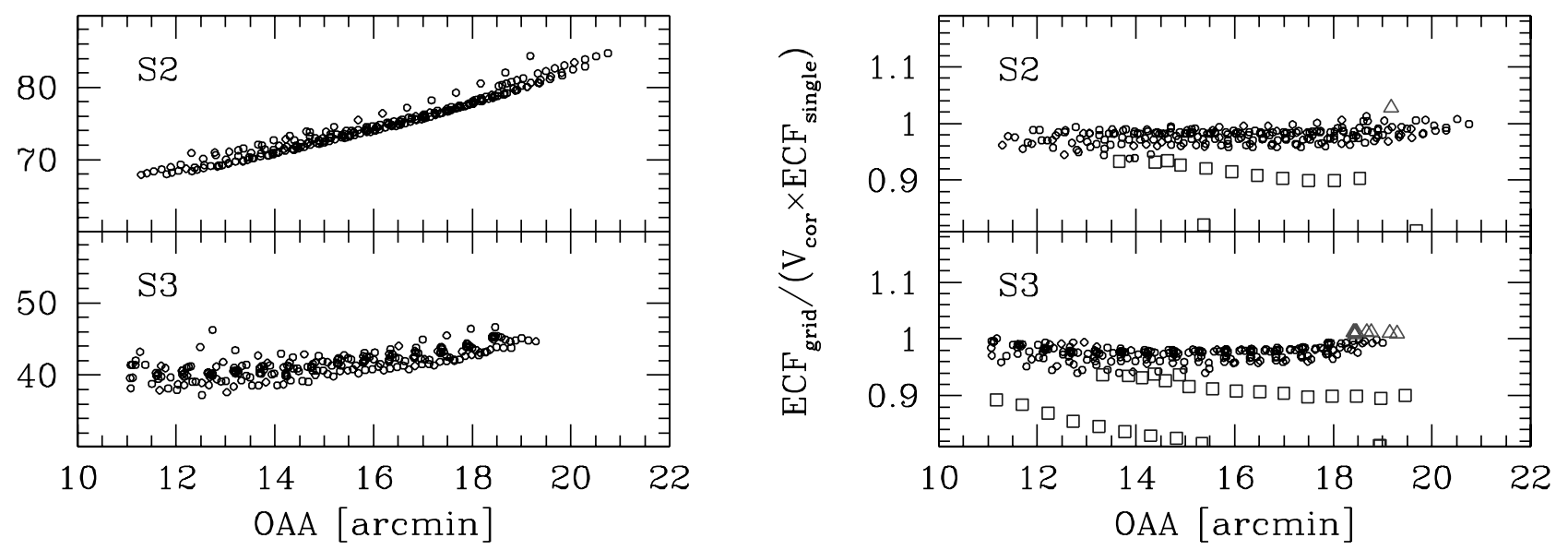

FIG. 8.- (Left) The spatial variations of $\mathrm{ECF}_{\text {grid }}$ in the $\mathrm{S}$ band as a function of off-axis angle in each CCD chip observed with ACIS-I including S2 and S3 CCD chips. The aim point of this observation is located on the $\mathrm{I} 3 \mathrm{CCD}$ chip, so the off-axis angle of $\mathrm{S} 2$ and $\mathrm{S} 3$ chips is large. $\mathrm{ECF}_{\text {grid }}$ varies by at most $\sim 25 \%$ depending on position in each CCD chip. $($ Right $)$ The ratios of $\mathrm{ECF}_{\text {grid }}$ to the vignetting-corrected $\mathrm{ECF}_{\text {single. }}$ Squares are caused by the exposure map defects such as a CCD chip edge or bad pixel strip effect. Triangles are caused by lower quantum efficiency at larger off-axis angles. The mean ratio is $1.00 \pm 0.02$ in the I0-I3 chips and $0.98 \pm 0.02$ and $0.97 \pm 0.03$ in the S2 and $\mathrm{S} 3$ chips. [See the electronic edition of the Supplement for a color version of this figure.]

observed ChaMP field for our simulations, rather than blank background sky fields, to investigate the effects of background counts and source confusion.

We used the active I0, I1, I2, and I3 CCD chips for ACIS-I, and I2, I3, S2, and S3 CCD chips for ACIS-S Chandra observations, and simulated 1000 artificial X-ray sources per Chandra observation. The number of detected artificial sources in each field depends on the effective exposure time and the observed region of the sky with various values of $N_{\mathrm{H}}$. On average, $11.4 \%$ of the 146,178 input artificial X-ray sources are detected in our simulations, for a total 16,676 artificial X-ray sources in 149 ChaMP fields. The number of detected artificial X-ray sources is 2.3 times the 7106 ChaMP sources in the same CCD chips and observations and statistically sufficient to estimate the properties of the ChaMP fields.

The form of the assumed number counts distribution is not critical to determine the detection probability, which is determined by the ratio of input to output numbers at a given flux (Vikhlinin et al. 1995; Kim \& Fabbiano 2003). The actual X-ray differential number counts are described by a broken/double power law with faint and bright slopes of $\sim-1.5$ and $\sim-2.5$, respectively (Yang et al. 2004; Basilakos et al. 2005; Chiappetti et al. 2005) in most energy bands; however, the break flux has not been well determined. Therefore, we assumed a cumulative number counts distribution with a single power law and a slope of -1 corresponding to a slope of -2 in the differential number counts, taking the 
TABLE 3

EnERgy Conversion FACTOR

\begin{tabular}{|c|c|c|c|c|c|c|c|c|c|c|c|c|c|c|c|c|c|c|c|}
\hline \multirow[b]{2}{*}{$\begin{array}{l}\text { Oвs. ID } \\
\text { (1) }\end{array}$} & \multirow[b]{2}{*}{$\begin{array}{l}\text { CCD ID } \\
\text { (2) }\end{array}$} & \multicolumn{6}{|c|}{$\Gamma_{\mathrm{ph}}=1.2$} & \multicolumn{6}{|c|}{$\Gamma_{\mathrm{ph}}=1.4$} & \multicolumn{6}{|c|}{$\Gamma_{\mathrm{ph}}=1.7$} \\
\hline & & $\begin{array}{l}\text { B } \\
\text { (3) }\end{array}$ & $\begin{array}{l}\text { S } \\
(4)\end{array}$ & $\begin{array}{l}\mathrm{H} \\
(5)\end{array}$ & $\begin{array}{l}\mathrm{Bc} \\
(6)\end{array}$ & $\begin{array}{l}\mathrm{Sc} \\
(7)\end{array}$ & $\begin{array}{l}\mathrm{Hc} \\
(8)\end{array}$ & $\begin{array}{l}\text { B } \\
(9)\end{array}$ & $\begin{array}{c}\mathrm{S} \\
(10)\end{array}$ & $\begin{array}{c}\mathrm{H} \\
(11)\end{array}$ & $\begin{array}{l}\mathrm{Bc} \\
(12)\end{array}$ & $\begin{array}{c}\mathrm{Sc} \\
(13)\end{array}$ & $\begin{array}{l}\mathrm{Hc} \\
(14)\end{array}$ & $\begin{array}{c}\mathrm{B} \\
(15)\end{array}$ & $\begin{array}{c}\mathrm{S} \\
(16)\end{array}$ & $\begin{array}{c}\mathrm{H} \\
(17)\end{array}$ & $\begin{array}{l}\mathrm{Bc} \\
(18)\end{array}$ & $\begin{array}{c}\mathrm{Sc} \\
(19)\end{array}$ & $\begin{array}{l}\mathrm{Hc} \\
(20)\end{array}$ \\
\hline $135 \ldots$. & 2 & 130.25 & 64.24 & 289.26 & 128.03 & 50.19 & 262.14 & 117.76 & 65.36 & 277.79 & 114.15 & 50.74 & 250.33 & 104.04 & 68.04 & 262.06 & 97.53 & 51.74 & 234.34 \\
\hline $135 \ldots \ldots \ldots \ldots$ & 3 & 31.44 & 65.01 & 289.92 & 129.12 & 50.77 & 263.01 & 118.98 & 66.21 & 278.41 & 115.23 & 51.36 & 251.20 & 105.31 & 69.03 & 262.70 & 98.60 & 52.44 & 235.21 \\
\hline $135 \ldots$ & 5 & 103.05 & 44.69 & 341.86 & 108.74 & 37.58 & 289.69 & 87.98 & 43.67 & 324.75 & 93.20 & .10 & 272.63 & 71.00 & 42.50 & 301.68 & 75.06 & 36.43 & 249.91 \\
\hline $35 \ldots$. & 6 & 115.00 & 56.38 & 259.13 & 113.61 & 44.20 & 35.68 & 103.57 & 57.09 & 249.59 & 101.04 & 44.53 & 225.69 & 90.79 & 58.94 & 236.46 & 85.88 & 5.16 & 212.07 \\
\hline $35 \ldots$ & 7 & 85.12 & 8.20 & 248.29 & 89.43 & 32.10 & 17.62 & 73.37 & 37.40 & 237.38 & 77.30 & 31.73 & 206.28 & 59.93 & 36.52 & 222.43 & 62.89 & 31.19 & 190.93 \\
\hline $25 \ldots .$. & 2 & 135.69 & 65.77 & 314.52 & 133.17 & 51.72 & 77.16 & 122.42 & 67.05 & 301.06 & 118.41 & 52.31 & 263.12 & 107.98 & 70.02 & 282.68 & 100.88 & 3.40 & 244.09 \\
\hline ...... & 3 & 136.58 & 66.61 & 311.79 & 133.90 & 52.20 & 76.84 & 123.44 & 67.96 & 298.62 & 119.24 & 52.85 & 263.16 & 109.16 & 71.07 & 280.62 & 101.81 & 54.01 & 244.65 \\
\hline ............ & 6 & 116.57 & 56.64 & 268.51 & 114.83 & 44.43 & 40.60 & 104.94 & 57.48 & 258.07 & 102.01 & 44.81 & 229.59 & 92.06 & 59.55 & 243.72 & 86.62 & 45.50 & 214.58 \\
\hline $25 \ldots \ldots$ & 7 & 87.06 & 9.22 & 250.62 & 90.66 & 32.64 & 19.17 & 75.33 & 38.53 & 240.01 & 78.57 & 2.33 & 208 & 61.90 & 37.82 & 225.51 & 64.19 & 1.90 & 193.17 \\
\hline 326 & 2 & 141.37 & 72.20 & 289.98 & 137.55 & 56.19 & 63.37 & 129.48 & 74.21 & 278.53 & 123.88 & 57.19 & 25 & 116.84 & 78.56 & 262.84 & 107.52 & 58.94 & 235.67 \\
\hline $326 \ldots$. & 3 & 142.52 & 72.95 & 290.89 & 138.63 & 56.76 & 264.47 & 130.64 & 75.04 & 279.41 & 124.94 & 57.80 & & 118.06 & 79.53 & 263.69 & 108.58 & 59.64 & 236.72 \\
\hline $326 \ldots$ & 5 & 120.60 & 54.50 & 343.34 & 121.62 & 43.95 & 291.69 & 105.60 & 54.40 & 326.20 & 105 & 43.84 & & 81 & 54.81 & 303.08 & 87.11 & 74 & 51.86 \\
\hline $326 \ldots \ldots .$. & 6 & 123.48 & 62.82 & 255.43 & 120.47 & 48.92 & 2. & 112.81 & 64.33 & 246.06 & 10 & 65 & & 101.26 & 67.67 & 233.17 & .65 & .94 & 209.66 \\
\hline $26 \ldots \ldots \ldots$ & 7 & 98.82 & 46.35 & 250.47 & 36 & 37.54 & 1 & 87.34 & 46.35 & 239.60 & 87 & & 20 & 5 & 46.85 & 224.73 & 73.11 & 7.58 & 194.26 \\
\hline 42. & 2 & 134.75 & 67.44 & 289.09 & 131.78 & 52.54 & 2.23 & 122.53 & 68.93 & 277.65 & 118.00 & 53 & 250 & 109.28 & 72.31 & 261.99 & 101.50 & 4.58 & 234.53 \\
\hline $342 .$. & 3 & 135.96 & 68.20 & 290.14 & 132.89 & 53.11 & 263.41 & 123.75 & 69.77 & 278.65 & 119.10 & 53.89 & 251.61 & 110.55 & 73.29 & 262.93 & 102.58 & 5.28 & 235.66 \\
\hline ......... & 5 & 110.56 & 48.78 & 342.63 & 114.09 & 40.15 & 290.59 & 95.47 & 48.13 & 325.50 & 98.40 & 39.82 & 273.51 & 78.48 & 47.57 & 302.36 & 80.02 & 9.38 & 250.76 \\
\hline $342 \ldots$ & 6 & 117.31 & 58.39 & 254.84 & 115.17 & 45.59 & 1.94 & 106.32 & 59.43 & 245.47 & 102.90 & 46.08 & 222. & 94.18 & 61.88 & 232.56 & 88.10 & 46.96 & 208.77 \\
\hline $342 \ldots \ldots \ldots$. & 7 & 91.03 & 41.60 & 249.81 & 93.99 & 34.36 & 219.79 & 79.37 & 41.14 & 238.95 & 81.85 & 34.16 & 208.54 & 66.07 & 40.82 & 224.08 & 67.39 & 33.91 & 193.32 \\
\hline 346....................... & 2 & 142.39 & 70.28 & 315.57 & 138.98 & 55.17 & 278.54 & 129.36 & 72.03 & 302.11 & 124.25 & 56.01 & 264.48 & 115.39 & 75.89 & 283.72 & 106.75 & 57.50 & 245.45 \\
\hline $346 \ldots .$. & 3 & 143.17 & 71.10 & 312.78 & 139.63 & 55.64 & 278.13 & 130.28 & 72.92 & 299.59 & 125.01 & 56.53 & 264.46 & 116.49 & 76.92 & 281.59 & 107.63 & 58.10 & 245.94 \\
\hline $346 \ldots . .$. & 6 & 122.63 & 60.78 & 269.13 & 120.00 & 47.52 & 241.52 & 111.24 & 62.04 & 258.69 & 107.23 & 48.11 & & 98.83 & 64.91 & 244.37 & 91.90 & 49.16 & 215.55 \\
\hline $346 \ldots$. & 7 & 94.67 & 43.72 & 251.38 & 96.25 & 35.56 & 220.19 & 83.06 & 43.46 & 240.76 & 84.06 & 35.41 & & 69.83 & 43.46 & 226.25 & 69.51 & 35.23 & 194.16 \\
\hline $363 \ldots$ & 1 & 71.64 & 85.40 & 373.31 & 167.13 & 66.33 & 335.14 & 156.41 & 87.78 & 356.94 & 149.76 & 67.53 & & 140.29 & 92.93 & 334.70 & 129.20 & 69.63 & 296.58 \\
\hline $363 \ldots$. & 2 & 140.46 & 71.54 & 289.81 & 136.75 & 55.67 & 263.18 & 128.52 & 73.48 & 278.36 & 123.06 & 56.63 & 251.40 & 115.80 & 77.69 & 262.70 & 106.68 & 58.33 & 235.49 \\
\hline$\ldots \ldots \ldots \ldots$ & 3 & 141.64 & 72.30 & 290.84 & 137.85 & 56.25 & 64.36 & 129.71 & 74.31 & 279.35 & 124.14 & 57.26 & 252.55 & 117.04 & 78.66 & 263.62 & 107.75 & 59.03 & 236.59 \\
\hline $363 \ldots \ldots$ & 6 & 122.66 & 62.21 & 255.43 & 119.76 & 4845 & 32.76 & 111.94 & 63.65 & 246.06 & 107.58 & 49.15 & 222.95 & 100.30 & 66.87 & 233.17 & 92.89 & 50.38 & 209.60 \\
\hline 363 & 7 & 97.79 & 45.71 & 250.42 & 99.07 & 37.10 & 220.62 & 86.29 & 45.65 & 239.55 & 86.89 & 37.07 & 209.38 & 73.24 & 46.03 & 224.68 & 72.33 & 37.07 & 194.16 \\
\hline 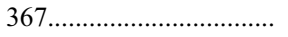 & 1 & 160.01 & 77.47 & 372.08 & 157.13 & 60.37 & 333.43 & 144.28 & 78.97 & 355.71 & 139.66 & 61.12 & 316.96 & 127.18 & 82.44 & 333.48 & 118.95 & 62.46 & 294.88 \\
\hline 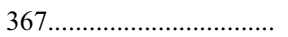 & 2 & 131.06 & 64.83 & 289.04 & 128.71 & 50.63 & 262.02 & 118.62 & 66.01 & 277.59 & 114.86 & 51.21 & 250.23 & 104.97 & 68.81 & 261.90 & 98.25 & 52.26 & 234.29 \\
\hline
\end{tabular}

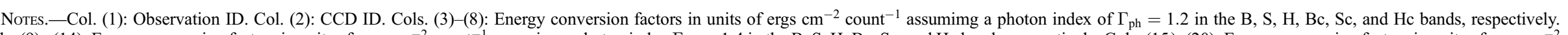

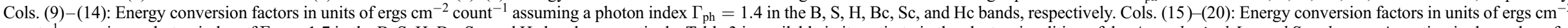

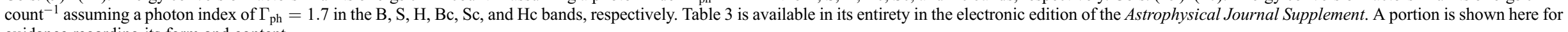
guidance regarding its form and content. 
TABLE 4

Definition of Source Flag

\begin{tabular}{|c|c|}
\hline Flag & Definition \\
\hline \multicolumn{2}{|r|}{ 1n: False X-ray sources } \\
\hline $11 \ldots \ldots \ldots$ & False source due to a hot pixel or by a bad bias value \\
\hline $12 \ldots \ldots \ldots$ & False source due to a bad column \\
\hline $13 \ldots \ldots \ldots$ & False source along the readout direction of a very strong source \\
\hline $14 \ldots \ldots \ldots$. & False source due to the FEP $0 / 3$ problem \\
\hline $15 \ldots \ldots \ldots$ & Double sources detected due to the PSF effect \\
\hline \multicolumn{2}{|r|}{$2 \mathrm{n}$ : It is not certain whether it is a valid X-ray source } \\
\hline $21 \ldots \ldots \ldots$ & V\&Ver found it might be a spurious source \\
\hline \multicolumn{2}{|r|}{ 3n: Source properties may be subject to a large uncertainty } \\
\hline $31 \ldots$ & Bad pixel/column exists within source extraction radius \\
\hline $37 \ldots \ldots \ldots$. & Pile-up (see Chandra POG 2004) \\
\hline $38 \ldots \ldots \ldots$ & Uncertain source position by flag $=015$ \\
\hline \multicolumn{2}{|r|}{ 5n: Other cases } \\
\hline $51 \ldots \ldots \ldots$ & Source is extended \\
\hline $52 \ldots \ldots \ldots$ & Same source candidate in multiple observations \\
\hline $53 \ldots \ldots \ldots$ & Target of observation \\
\hline $54 \ldots$ & X-ray jet \\
\hline $55 \ldots$ & Variable source (see Paper I) \\
\hline \multicolumn{2}{|r|}{$6 \mathrm{n}$ : Flagged by the automatic pipeline xapphot } \\
\hline $61 \ldots \ldots \ldots$ & Source region falls near the edge of the chip \\
\hline $62 \ldots$ & Small portion of source region overlaps with nearby point source \\
\hline $63 \ldots \ldots \ldots$ & Large portion of source region overlaps with nearby point source \\
\hline $64 \ldots \ldots \ldots$ & Small portion of source region overlaps with nearby extended source \\
\hline $65 \ldots \ldots \ldots$ & Large portion of source region overlaps with nearby extended source \\
\hline $66 \ldots \ldots \ldots$ & Background region overlaps with a nearby point source \\
\hline $67 \ldots \ldots \ldots$ & Background region overlaps with a nearby extended source \\
\hline $68 \ldots \ldots \ldots$ & Source region falls inside the extended source \\
\hline
\end{tabular}

average of the faint and bright slopes from the literature, in the $0.3-8 \mathrm{keV}$ band. The flux of an artificial source was randomly selected from the assumed number counts distribution in a flux range of $5 \times 10^{-16}-5 \times 10^{-10} \mathrm{erg} \mathrm{cm}^{-2} \mathrm{~s}^{-1}$, and the MARX generated the artificial sources with a flux range of $8 \times 10^{-17}-2 \times$ $10^{-11} \mathrm{erg} \mathrm{cm}^{-2} \mathrm{~s}^{-1}$ including the Poisson uncertainty of the input source counts. The flux range of the detected artificial sources spans $1 \times 10^{-17}-2 \times 10^{-11} \mathrm{erg} \mathrm{cm}^{-2} \mathrm{~s}^{-1}$, which covers the flux range of the actual ChaMP X-ray point sources, $1 \times 10^{-17}-6 \times$ $10^{-12} \mathrm{erg} \mathrm{cm}^{-2} \mathrm{~s}^{-1}$.

We assume a power-law spectrum with a photon index of $\Gamma_{\mathrm{ph}}=$ 1.7, because the ChaMP X-ray point sources with $\mathrm{S} / \mathrm{N}>1.5$ have $\Gamma_{\text {ph }}=1.5-2$ (Kim et al. 2004b; see Figs. 20 and 21 in this paper). We note that these sources cover a flux range of $4 \times 10^{-16}-2 \times$ $10^{-12}(0.5-2 \mathrm{keV})$ and $2 \times 10^{-15}-7 \times 10^{-12}(2-8 \mathrm{keV})$ in ergs $\mathrm{cm}^{-2} \mathrm{~s}^{-1}$, respectively. Tozzi et al. (2006) performed X-ray spectral analysis for $82 \mathrm{X}$-ray bright sources in the CDF-S, and they found that the weighted mean value for the slope of the power-law spectrum is $\left\langle\Gamma_{\mathrm{ph}}\right\rangle \simeq 1.75 \pm 0.02$.

The flux range of these bright sources in the CDF-S overlaps with the faint flux end of the ChaMP sources, therefore, we assumed that the faint ChaMP sources $(\mathrm{S} / \mathrm{N}<1.5)$ also have a photon index of $\Gamma_{\mathrm{ph}} \sim 1.7$. We assumed Galactic absorption, $N_{\mathrm{H}}$ (Stark et al. 1992), for each observation; however, we did not include intrinsic absorption in the artificial source spectrum. The spectrum of each X-ray point source was generated using the XSPEC ${ }^{18}$ package.

\footnotetext{
18 See http://xspec.gsfc.nasa.gov/
}

The position of an artificial source was randomly selected on each CCD chip, but it was rejected if the source area at a given random position had an exposure map value with less than $10 \%$ of the maximum. This requirement is identical to that in the ChaMP $\mathrm{X}$-ray point source reduction procedure. To avoid overcrowding of the artificial sources, $\sim 250$ artificial sources per CCD were divided into several groups to be added into the observed image: while we did not allow the artificial X-ray point sources to overlap one another, we allowed overlap between artificial and real X-ray sources to provide an estimate of source confusion in each observed field. This resulted in $\sim 10(\sim 20)$ simulated images per ACIS-I (ACIS-S) CCD, corresponding to $\sim 10,500$ CCD images (event files) to run through wavdetect (xapphot). Since $\sim 11.4 \%$ of the artificial sources are detected on average, we added only $\sim 1.5$ artificial sources to each simulated image. The net counts of the overlapping artificial sources with real sources were corrected following the overlapping source correction methods described in $\S$ 3.2.2.

To correct the temporal QE degradation of Chandra, ${ }^{19}$ we used $\mathrm{ECF}_{\text {single }}$ for each observation, as described in $\S 3.2 .4$. However, because of the mismatch between calibration data used in MARX version 4.0.8 and our analysis, there is a slight difference in the count-flux conversion. Thus, we performed a set of test simulations for each CCD chip and observation to correct this mismatch and then renormalized the MARX output by as much as $10 \%$ per CCD chip in each observation. After generating and adding artificial X-ray point sources into observed X-ray images, we detected them and extracted their source properties with exactly the same techniques as used in the ChaMP X-ray point source catalog.

\subsection{Positional Uncertainty}

\subsubsection{Empirical Equation of Positional Uncertainty}

The positional uncertainty of Chandra X-ray sources is a function of source counts, off-axis angle, and background counts. To estimate the positional uncertainty in the ChaMP fields, we investigated the offset between input and detected position for the artificial sources. To estimate the positional offsets of artificial sources, first we excluded the observed X-ray sources in simulated images to avoid the confusion caused by a mixture of observed and artificial X-ray sources. Second, we matched the input and detected artificial sources within twice the input source radius. The nearest object in this matching radius was assigned as a matched pair. An object with more than one match was assigned as a pair with the nearest neighbor. We then carefully performed a visual inspection to reject incorrectly matched sources. In the top panel of Figure 9, we display the positional offset of the artificial X-ray sources split into three source count categories as a function of off-axis angle. Since the source position is determined by wavdetect, we used the source counts measured by wavdetect rather than by our aperture photometry xapphot. It appears that the positional offsets exponentially increase with off-axis angle and decrease as the source count increases with a power-law form.

Applying the exponential function and the power law, we derive empirical equations for the positional uncertainty of ChaMP $\mathrm{X}$-ray point sources. At a $95 \%$ confidence level, these are

$\log \mathrm{PU}=$

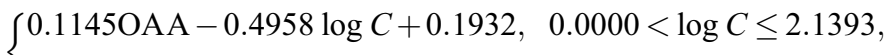
$\{0.0968 \mathrm{OAA}-0.2064 \log C-0.4260, \quad 2.1393<\log C \leq 3.3000$.

${ }^{19}$ See CXC Memo on 2002 July 29 (http://cxc.harvard.edu/cal/Acis/Cal_ prods/qeDeg/index.html). 

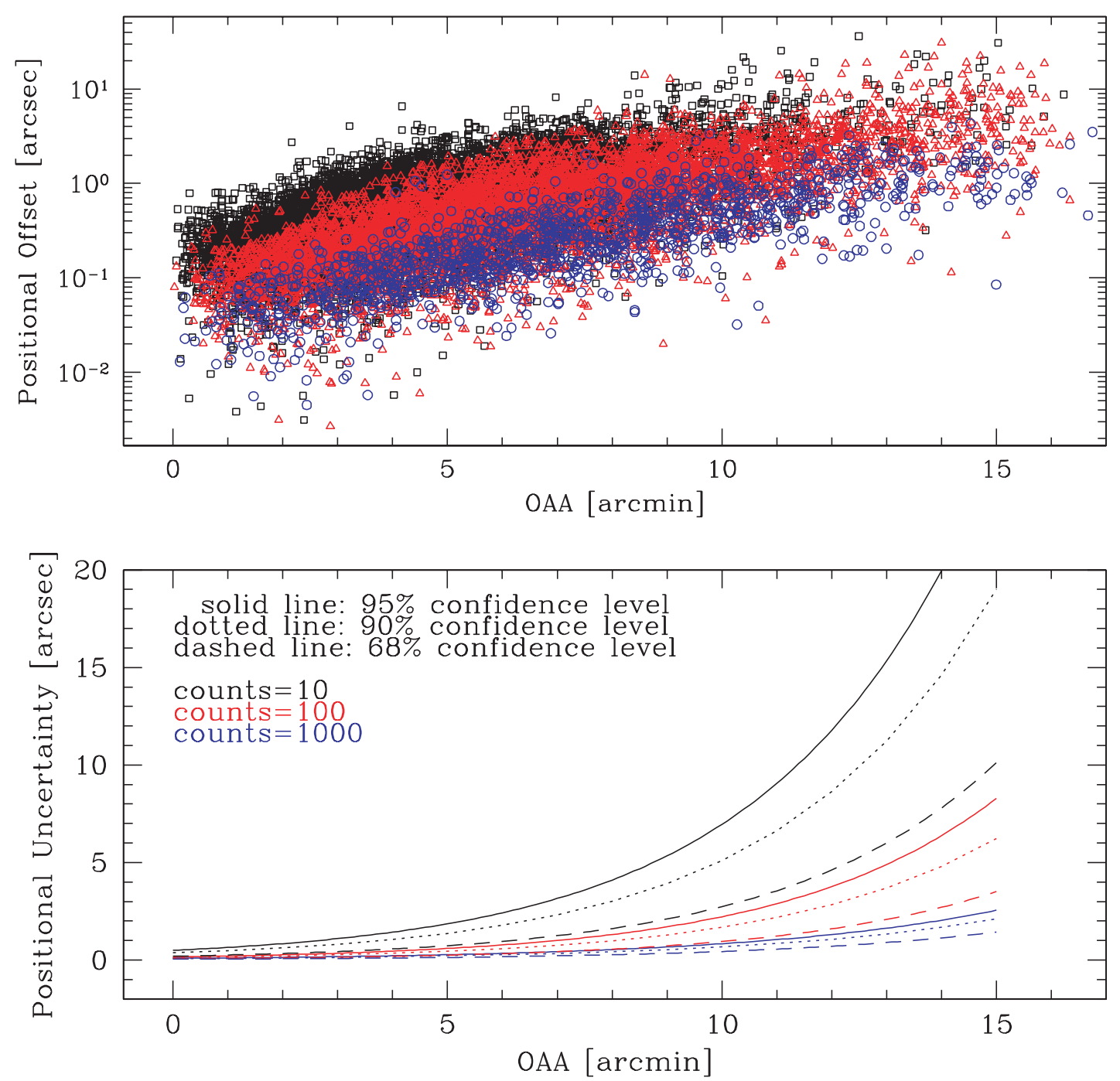

FIG. 9.-Top: Positional offset of the artificial sources in three source count ranges as a function of off-axis angle. The black squares, red triangles, and blue circles represent artificial sources with count ranges of $0<\log C<1.2,1.2<\log C<2$, and $\log C>2$, respectively. The positional offset exponentially increases with offaxis angle and decreases as the source counts increase with a power-law form. Bottom: The positional uncertainties from the derived equations (see eqs. [12], [13], and [14] in $\S 4.2 .1)$ as a function of off-axis angle for 10 source counts (black), 100 source counts (red), and 1000 source counts (blue), respectively. The solid, dotted, and dashed lines represent the positional uncertainty at $95 \%, 90 \%$, and $68 \%$ confidence levels, respectively.

At a $90 \%$ confidence level:

$\log \mathrm{PU}=$

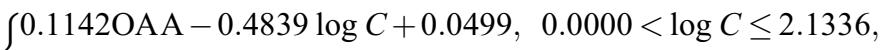

$\{0.0989$ OAA $-0.2027 \log C-0.5500,2.1336<\log C \leq 3.3000$.

At a $68 \%$ confidence level:

$\log \mathrm{PU}=$

$\{0.1137$ OAA $-0.4600 \log C-0.2398, \quad 0.0000<\log C \leq 2.1227$,

$\{0.1031$ OAA $-0.1945 \log C-0.8034,2.1227<\log C \leq 3.3000$.

Here positional uncertainty, PU, is in arcseconds, and off-axis angle, OAA, is in arcminutes. Source counts, $C$, are as extracted by wavdetect. The above equations are valid for the ChaMP
$\mathrm{X}$-ray point sources with an off-axis angle $\mathrm{OAA} \lesssim 15^{\prime}$ and source counts $\log C \lesssim 3.3$. For ChaMP X-ray point sources located at off-axis angle larger than $15^{\prime}$, the positional uncertainties were assigned to be $60^{\prime \prime}$. In the bottom panel of Figure 9, using equations (12)-(14), we display the positional uncertainties as a function of off-axis angle for three different source counts. Figure 10 shows the number distributions of positional uncertainty of the ChaMP X-ray point sources from equations (12)-(14). For 68\%, $90 \%$, and $95 \%$ confidence level positional uncertainty distributions, the medians are $0.7^{\prime \prime} \pm 0.45^{\prime \prime}, 1.3^{\prime \prime} \pm 0.8^{\prime \prime}$, and $1.8^{\prime \prime} \pm$ $1.1^{\prime \prime}$, respectively.

We also investigated the dependence of the positional uncertainties on the background counts; however, it is negligible in the ChaMP sample. Since we excluded high-background regions such as the Galactic plane, the background counts per unit pixel of ChaMP X-ray sources are only $3 \times 10^{-4}-9 \times 10^{-2}$ counts pixel $^{-1}$ and there are no significant background fluctuations in these fields. However, the background effect should be carefully considered to estimate the positional uncertainties of X-ray sources when the background fluctuations are severe, such as in the Galactic plane. 


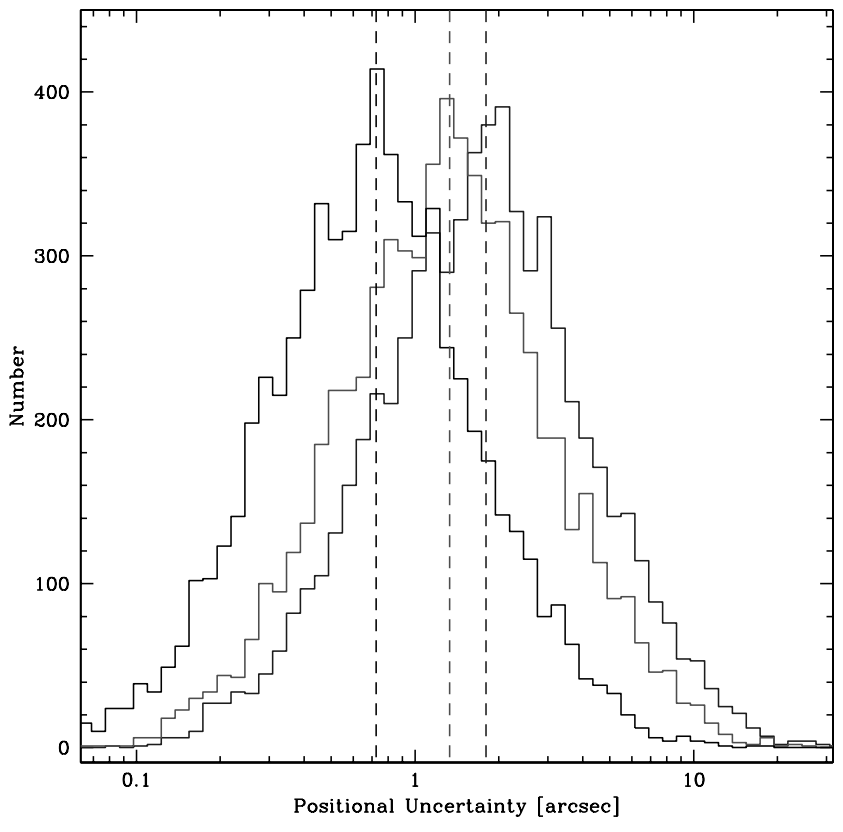

FIG. 10.-Number distributions of positional uncertainty of all ChaMP X-ray point sources estimated from our empirical positional uncertainty equations. The three histograms show the $68 \%, 90 \%$, and $95 \%$ confidence level of positional uncertainty distributions, respectively. The median positional uncertainties for all sources are plotted as dashed lines and shown at $0.7^{\prime \prime} \pm 0.45^{\prime \prime}, 1.3^{\prime \prime} \pm 0.8^{\prime \prime}$, and $1.8^{\prime \prime} \pm 1.1^{\prime \prime}$, respectively. [See the electronic edition of the Supplement for a color version of this figure.]

\subsubsection{Astrometry}

To ensure accurate absolute positions for the ChaMP X-ray point sources, we apply the standard Chandra aspect offsets. ${ }^{20}$ For the ChaMP data set, the magnitude of the mean aspect offset correction is $\sim 0.5^{\prime \prime}$, and the maximum is $\sim 2.7^{\prime \prime}$ (for OBSID 521). To further check the absolute positional accuracy of our ChaMP $\mathrm{X}$-ray point sources, we matched the ChaMP X-ray sources with the SDSS-DR3 ${ }^{21}$ (hereafter SDSS) optical objects, for which the absolute positional uncertainty is less than $0.5^{\prime \prime}$. Sixty of the 149 ChaMP fields overlap with the SDSS sky regions. Using the 95\% confidence level positional uncertainty equation (eq. [12] in $\S 4.2 .1$ ), over $2^{\prime \prime}<$ radius $<12.33^{\prime \prime}$, we searched the SDSS optical candidates of the ChaMP X-ray sources. The minimum searching radius is large enough not to miss probable partners, and the maximum searching radius corresponds to half the mean separation of the SDSS objects, thereby, reducing the number of randomly matched objects.

Some X-ray sources have more than one SDSS candidate counterpart. To decide the most appropriate SDSS counterpart for these $\mathrm{X}$-ray sources, we consider their X-ray and optical properties in addition to their offsets. The X-ray sources have a typical relation between X-ray flux and optical magnitude (Manners et al. 2003; Green et al. 2004). We applied the normalized distance $D_{n}$ between ChaMP and SDSS sources with positional uncertainty PU and X-ray to optical flux ratio $f_{\mathrm{X}} / f_{r}$ as follows:

$$
\begin{gathered}
D_{n}=D / \text { PU, } \\
\log \left(f_{\mathrm{X}} / f_{r}\right)=\log \left(f_{\mathrm{X}}\right)+5.41+0.4 \times m_{r},
\end{gathered}
$$

where $D$ is the distance between the ChaMP and the SDSS counterpart, $f_{\mathrm{X}}$ is the X-ray flux in the $\mathrm{Sc}$ band, and $m_{r}$ is the visual

\footnotetext{
${ }^{20}$ See http://cxc.harvard.edu/cal/ASPECT/.

21 See http://www.sdss.org/dr3/.
}
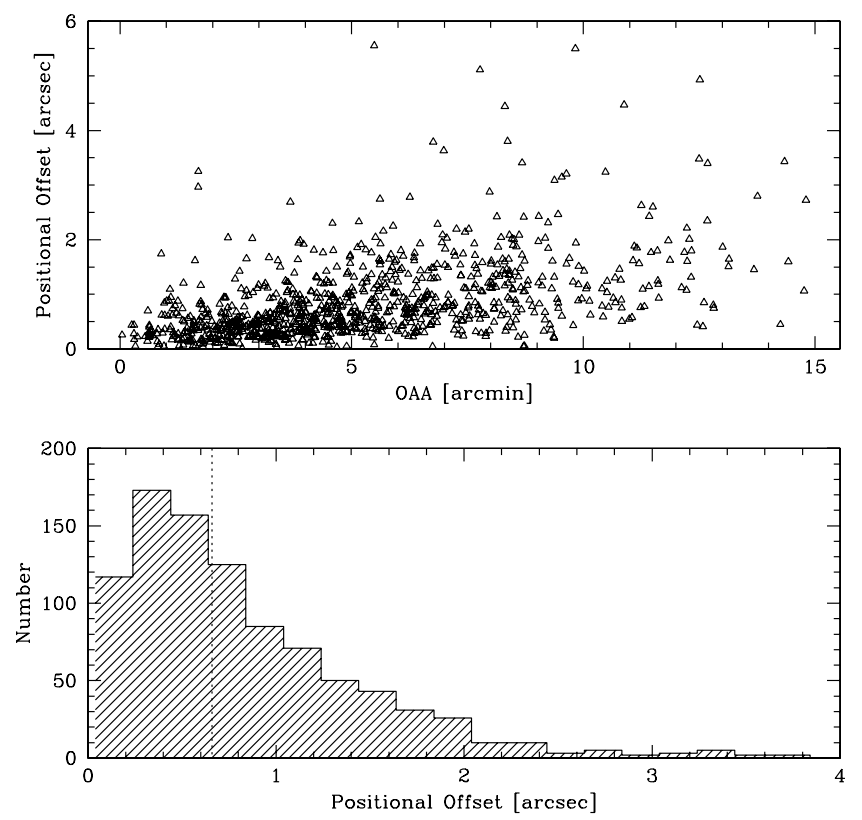

FIG. 11.-Top: Positional offset between the matched ChaMP and SDSS sources as a function of off-axis angle. Bottom: Number distribution of the positional offset between the matched ChaMP and the SDSS sources. The median positional offset is $0.7^{\prime \prime} \pm 0.4^{\prime \prime}$ and is denoted by the dotted line.

magnitude in the $\mathrm{r}$ band. First, for objects with one counterpart and with OAA $<6^{\prime}$, we calculated the average of $D_{n}$ and $\log \left(f_{\mathrm{X}} / f_{r}\right)$. Second, for objects with multiple counterparts, we calculated the standard deviations of $D_{n}$ and $\log \left(f_{\mathrm{X}} / f_{r}\right)$ relative to the average $D_{n}$ and $\log \left(f_{\mathrm{X}} / f_{r}\right)$ and introduced a likelihood as follows:

$$
L=\sqrt{\Delta_{\log \left(f_{\mathrm{X}} / f_{r}\right)}^{2}+\Delta_{D_{n}}^{2}}
$$

where $\Delta_{x} \equiv(x-\bar{x}) / \sigma_{x}$. Finally, we chose the counterpart with the lowest $L$ among the multiple counterparts as the most appropriate counterpart.

We also calculated the average $\log \left(f_{\mathrm{X}} / f_{r}\right)$ as a function of optical color and optical size. We classified the SDSS sources into four groups and recalculated the average of $\log \left(f_{\mathrm{X}} / f_{r}\right)$ for each group. The four groups are resolved (galaxies), unresolved with $u-g<0.6$ (UVX QSOs), unresolved with $g-r>1.2$ (M stars), and all other unresolved. We then redetermined the SDSS counterpart for each X-ray source, after repeating the above procedures. Except for 20 of 1600 pairs, the matching results were same. To confirm the matching results, we performed a visual inspection for all matched objects. In the top panel of Figure 11, the positional offsets between the ChaMP and the SDSS source are plotted as a function of off-axis angle. The positional offset increases exponentially with off-axis angle. The bottom panel shows the number distribution of the positional offsets between the matched ChaMP and SDSS sources. The median positional offset of confirmed $\sim 900$ matched sources is $0.7^{\prime \prime} \pm 0.4^{\prime \prime}$.

\subsection{ChaMP Source Reliability}

To understand the source reliability of the ChaMP point source catalog, we have investigated the detection probability, count recovery rate, false source rate, and flux limit of each ChaMP field using the simulation results.

\subsubsection{Detection Probability}

The detection probability is determined by the number ratio of detected artificial sources to input artificial sources. Since the 


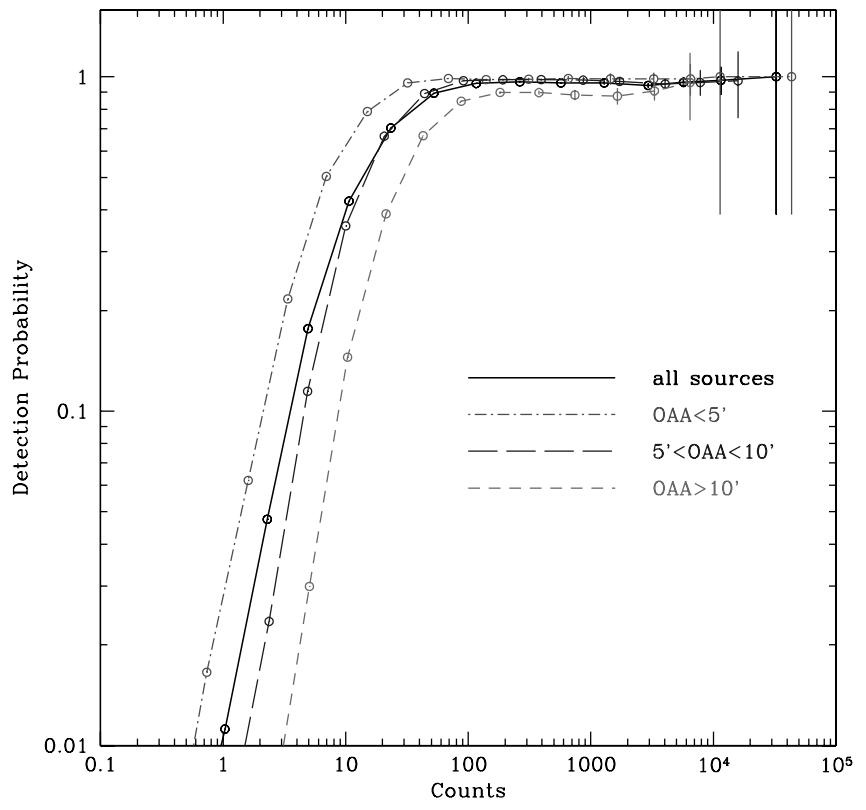

FIG. 12.-Detection probability of a source in the ChaMP catalog as a function of B band counts. The thick solid line represents the detection probability of all sources. The dot-dashed, long-dashed, and short-dashed lines denote the detection probability of sources located at off-axis angles, OAA $<5^{\prime}, 5^{\prime}<\mathrm{OAA}<10^{\prime}$, and $\mathrm{OAA}>10^{\prime}$, respectively. As expected, the detection probability decreases as the off-axis angle increases. [See the electronic edition of the Supplement for a color version of this figure.]

sensitivity of the Chandra CCD chip varies spatially, the detection probability is a function of off-axis angle as well as a function of source counts. Figure 12 shows the detection probability of a source as a function of the B-band counts depending on offaxis angle. The detection probability decreases as the source counts decrease, and as the off-axis angle increases because the sensitivity of the Chandra CCD chip decreases as the off-axis angle increases. Sources with counts $>30$ and off-axis angle $<5^{\prime}$ are detected with greater than $95 \%$ probability. We note that the detection probability as a function of flux may vary with source spectral shape if the source is quite different from our assumed spectral shape $\left(\Gamma_{\mathrm{ph}}=1.7\right)$.

\subsubsection{False Source Rate}

We also investigated the probability of false sources in the ChaMP catalog. With simulated images, we found that false sources are $\sim 1 \%$ of the total detected sources. Of these spurious sources, $80 \%$ have counts less than $\sim 30$. Figure 13 shows the false source detection rate as a function of the B-band source counts (top) and off-axis angle (bottom). The dashed lines indicate the best linear least-squares fitting results:

False source rate $(C)=-0.05( \pm 0.00) C+1.92( \pm 0.04)$,

False source rate $(\mathrm{OAA})=0.15( \pm 0.02) \mathrm{OAA}-0.01( \pm 0.18)$,

where OAA is in units of arcminutes and $C$ is the source counts extracted by wavdetect. The false source detection rate increases with decreasing source counts and increasing off-axis angle. To derive equation (18), we used false sources with $C<40$, and for equation (19) we used all false sources.
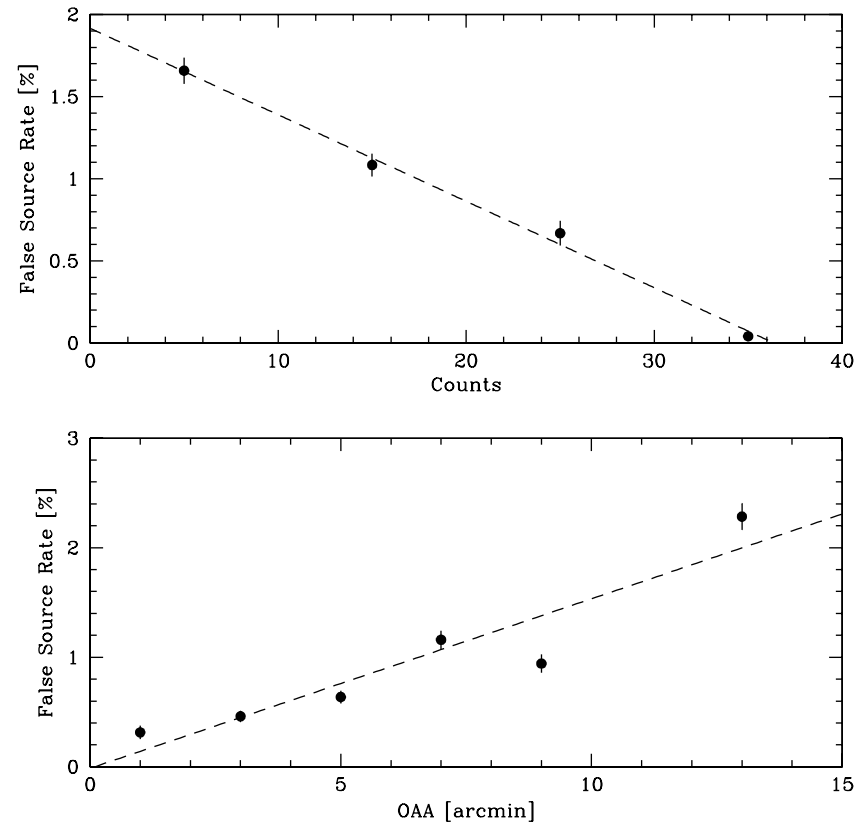

FIG. 13.-False source detection rate as a function of source counts in the B-band extracted by wavdetect (top) and off-axis angle (bottom). $\sim 1 \%$ of the total detected sources are spurious sources, and $80 \%$ of spurious sources have counts less than $\sim 30$. The false source detection rate increases as the source counts decrease and as the off-axis angle increases. The dashed lines indicate the best linear least-squares fit results (see $\S$ 4.3.2).
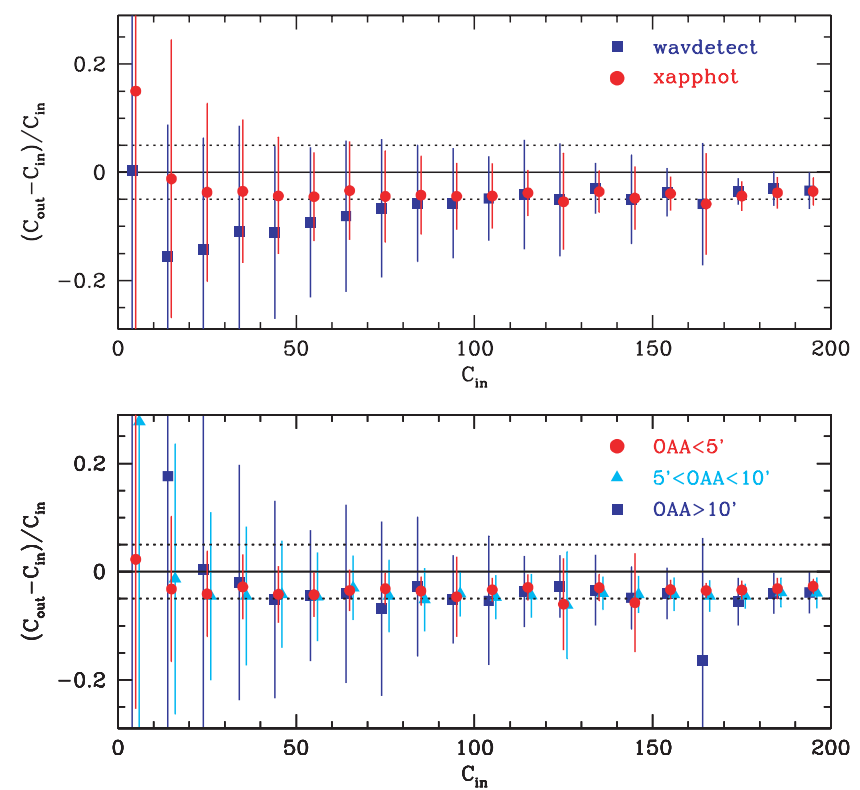

FIG. 14.-Count recovery rate as a function of input counts. Top: Comparison of the count recovery rate from xapphot (red circles) with that from wavdetect (blue squares). Both xapphot and wavdetect recover the true counts well (96\% $\pm 1 \%$ level for xapphot and $94 \% \pm 3 \%$ level for wavdetect); however, for source counts fainter than $\sim 50$, wavdetect recovers only $87 \% \pm 2 \%$ of the true counts. Note that Eddington bias is visible in the first points of both wavdetect and xapphot count recovery rates. Bottom: The count recovery rates of sources with $\mathrm{OAA}<5^{\prime}$ (red circle), $5^{\prime}<\mathrm{OAA}<10^{\prime}$ (cyan triangle), and OAA $>10^{\prime}$ (blue square), respectively. Source counts are extracted using xapphot. As the offaxis angle increases, the uncertainty in the count recovery rate increases. 


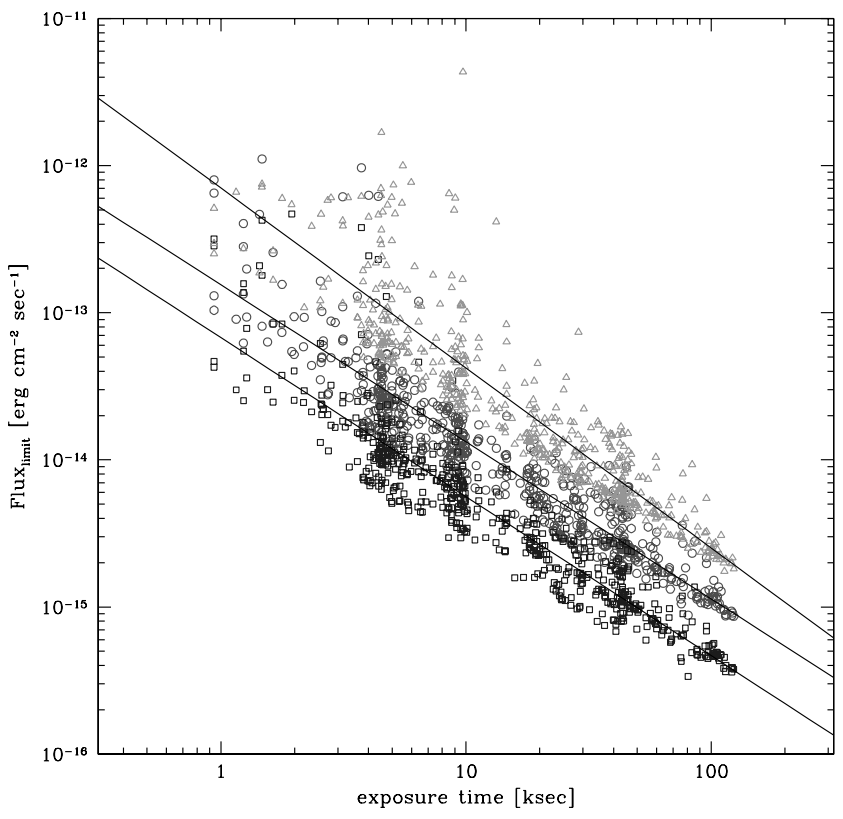

Fig. 15.-Flux limits of detected X-ray point sources in the ChaMP fields as a function of net exposure time. Four CCD chips (I0, I1, I2, and I3 for ACIS-I observations, and I2, I3, S2, and S3 for ACIS-S observations) per ChaMP field and the 130 ChaMP fields were used. Detected artificial X-ray point sources with $\mathrm{S} / \mathrm{N}>$ 2.0 are selected in each CCD chip, and their minimum flux is defined as the flux limit of that $\mathrm{CCD}$ chip. Circles, squares, and triangles represent the $\mathrm{Bc}, \mathrm{Sc}$, and $\mathrm{Hc}$ bands, respectively. The solid lines represent the best linear least-squares fit results in each energy band. The scatter is caused by the varying sensitivity and detection probability of each CCD chip and observation. A photon index of $\Gamma_{\mathrm{ph}}=1.7$ was assumed. [See the electronic edition of the Supplement for a color version of this figure.]

\subsubsection{Counts Recovery Rate}

To confirm the quality of our photometry, we investigated the count recovery rate defined by the ratio of the difference between input counts and output counts $\left(C_{\text {out }}-C_{\text {in }}\right)$ to input counts $\left(C_{\text {in }}\right)$. First, we compared the photometry results from xapphot (aperture photometry) and wavdetect. The top panel of Figure 14 shows the count recovery rate using xapphot (red circles) and using wavdetect (blue squares) as a function of input counts. The count recovery rate of xapphot is $96 \% \pm 1 \%$, regardless of input counts. This is very close to that expected, given that our choice of the source extraction radius corresponds to the $95 \%$ encircled energy. We note that our count recovery rate agrees with that of Tozzi et al. (2001) in which they applied aperture photometry to the CDF-S sources with the source extraction region defined as a circle of radius $R_{s}=2.4 \mathrm{FWHM}$, where FWHM is modeled from the PSF. While the wavdetect-determined count recovery rate is $96 \% \pm 2 \%$ for bright sources (counts $>50$ ), it underestimates the counts for sources with counts $<70$. For example, wavdetect recovers $87 \% \pm 2 \%$ for sources with input counts $\lesssim 50$. This is primarily because wavdetect uses a smaller source extraction radius for fainter sources.

Second, we have investigated the count recovery rate of xapphot depending on the off-axis angle in the bottom panel of Figure 14. The xapphot recovers source counts well regardless of the off-axis angle; however, the statistical errors of the count recovery rate increase as the off-axis angle increases, since the background fluctuations affect off-axis sources more severely than on-axis sources due to the larger source and background extraction regions.

\subsubsection{Flux Limit and Exposure Time}

Using four CCD chips (I0, I1, I2, and I3 for ACIS-I observations, and I2, I3, S2, and S3 for ACIS-S observations) per
ChaMP field, we derived the relation between flux limit and exposure time of the observation in each energy band. The detected artificial sources with $\mathrm{S} / \mathrm{N}>2.0$ have been selected in each CCD chip, and their minimum flux is defined as the flux limit of that CCD chip. Figure 15 shows the flux limits of detected artificial sources in the ChaMP fields as a function of the exposure time. The best linear least-squares fit results of the relation between flux limits and exposure times in each energy band are as follows:

$$
\begin{aligned}
\log F_{\text {limit }, \mathrm{B}} & =-1.04( \pm 0.02) \log \mathrm{ET}-12.87( \pm 0.03), \\
\log F_{\text {limit }, \mathrm{S}} & =-1.06( \pm 0.02) \log \mathrm{ET}-13.10( \pm 0.02), \\
\log F_{\text {limit } \mathrm{H}} & =-1.23( \pm 0.03) \log \mathrm{ET}-12.08( \pm 0.04), \\
\log F_{\text {limit }, \mathrm{Bc}} & =-1.06( \pm 0.02) \log \mathrm{ET}-12.81( \pm 0.02), \\
\log F_{\text {limit } \mathrm{Sc}} & =-1.08( \pm 0.02) \log \mathrm{ET}-13.17( \pm 0.02), \\
\log F_{\text {limit } \mathrm{Hc}} & =-1.22( \pm 0.03) \log \mathrm{ET}-12.15( \pm 0.03),
\end{aligned}
$$

where ET is the net exposure time of each CCD chip in units of ks after excluding any background flares (see Paper I) and the flux is estimated assuming a photon index of $\Gamma_{\mathrm{ph}}=1.7$. The scatter of the relation is caused by the varying sensitivity and detection probability of each CCD chip and OBSID. These equations give us a representative for the flux limit of $\mathrm{X}$-ray sources depending on their exposure time in the $\mathrm{B}, \mathrm{S}, \mathrm{H}, \mathrm{Bc}, \mathrm{Sc}$, and $\mathrm{Hc}$ bands in the ChaMP fields.

\section{ChaMP X-RAY POINT SOURCE CATALOGS}

\subsection{Catalogs}

We found 7365 X-ray point sources in 149 ChaMP fields, after excluding false sources (flags 11-21) and sources located close to the CCD chip edges (flag 61) (see Table 4 for flag definitions). The 102 target point sources (flag 53) are included in the catalog for completeness. We note that, for scientific analysis, target sources need to be carefully handled depending on their own scientific goals because they are not random sources. For example, we excluded the target sources to determine the X-ray point source number counts (Kim et al. 2004b; Kim et al. 2006b).

Since 35 of the 149 ChaMP fields partly overlap on the sky, as seen in the eleventh column of Table 1, there are sources observed more than once in these overlapping fields. For simplicity and flexible usage, we present the sources in the overlapping fields in separate tables (Tables 5-11): e.g., for the X-ray number counts research, we used only the main ChaMP catalog to derive the sky coverage avoiding complex overlapping fields (Kim et al. 2006b). In the main ChaMP tables (Table 5, Table 8, and Table 10), we present all sources in fields observed once and those in the overlapping fields with the longest exposure time. In the supplementary ChaMP tables ( Table 6, Table 9, and Table 11), we present the sources in the overlapping fields with shorter exposure times. The main ChaMP tables list $6512 \mathrm{X}$-ray point sources in $130 \mathrm{ChaMP}$ fields and the supplementary ChaMP tables list 853 sources in 19 ChaMP fields. Tables 5 and 6 contain the source position, positional uncertainty, off-axis angle, source radius, effective exposure time, and flag. Tables 8 and 9 give the photometry of the $\mathrm{X}$-ray point sources in eight X-ray energy bands. Tables 10 and 11 list the hardness ratio and colors such as C21 and C32 of the X-ray point sources. In Table 7, we list the same source candidates in the overlapping fields (453 pairs/triples of 926 sources) with their observation date, source counts, count rates, and positional uncertainties. We note that these candidates are identified only by their positions, matching sources in the overlapping fields within 
TABLE 5

The Main ChaMP X-Ray Point Sources

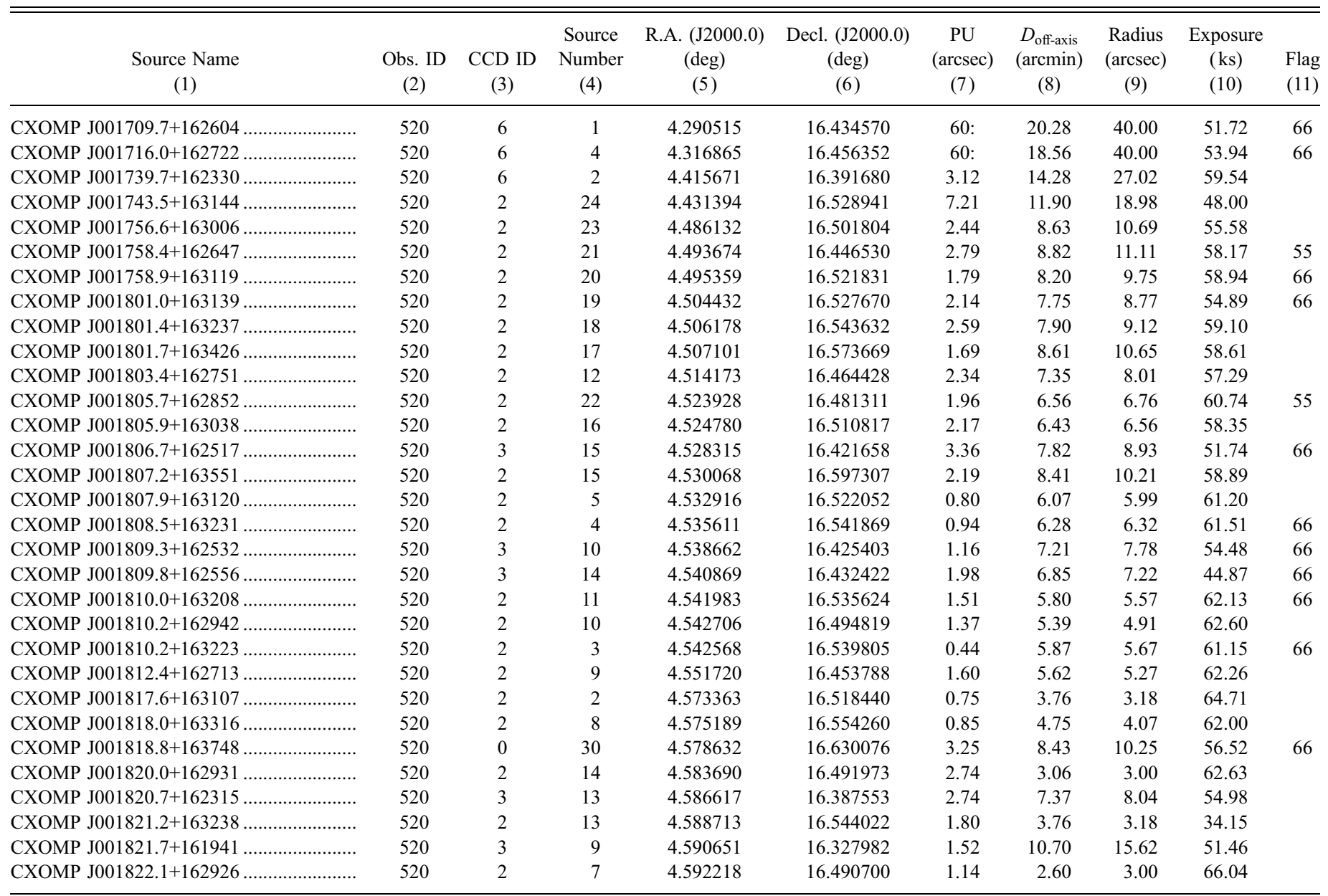

Notes.-Col. (1): ChaMP source name given by right ascension in units of hours, minutes, and seconds, and declination in units of degrees, arcminutes, and arcseconds in J2000.0. Col. (2): Observation ID. Col. (3): CCD ID (0-3 for ACIS-I and 4-9 for ACIS-S observation). Col. (4): Source number assigned by wavdetect as marked in the public X-ray image of the ChaMP Web site (http://hea-www.harvard.edu/CHAMP). Cols. (5) and (6): Right ascension and declination in units of degrees after refinement and aspect correction. Col. (7): Positional uncertainty in units of arcseconds estimated by the 95\% confidence level of the empirical formula (see eq. [12] in $\S 4.2$ ). Col. (8): Off-axis angle from the aim point of the observation in units of arcminutes. Col. (9): Source extraction radius in units of arcseconds (The 95\% encircled energy radius at $1.5 \mathrm{keV}$. See $\S 3.2 .1$ ). Col. (10): Mean effective exposure time in units of ks after vignetting correction (see $\S 3.2 .4)$. Col. (11): Source flag (see Table 4). Table 5 is available in its entirety in the electronic edition of the Astrophysical Journal Supplement. A portion is shown here for guidance regarding its form and content. 
TABLE 6

The Supplementary ChaMP X-Ray Point Sources

\begin{tabular}{|c|c|c|c|c|c|c|c|c|c|c|}
\hline $\begin{array}{c}\text { Source Name } \\
\text { (1) }\end{array}$ & $\begin{array}{l}\text { Obs. ID } \\
\text { (2) }\end{array}$ & $\begin{array}{l}\text { CCD ID } \\
\text { (3) }\end{array}$ & $\begin{array}{c}\text { Source } \\
\text { Number } \\
\text { (4) }\end{array}$ & 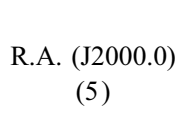 & $\begin{array}{c}\text { Decl. (J2000.0) } \\
\quad(6)\end{array}$ & $\begin{array}{c}\mathrm{PU} \\
(\operatorname{arcsec}) \\
(7)\end{array}$ & $\begin{array}{c}D_{\text {off-axis }} \\
\text { (arcmin) } \\
(8)\end{array}$ & $\begin{array}{c}\text { Radius } \\
(\operatorname{arcsec}) \\
(9)\end{array}$ & $\begin{array}{c}\text { Exposure } \\
(\mathrm{ks}) \\
(10)\end{array}$ & $\begin{array}{l}\text { Flag } \\
(11)\end{array}$ \\
\hline CXOMP J005622.4-272223 ……............ & 2242 & 3 & 2 & 14.093514 & -27.373174 & 6.50 & 12.31 & 20.13 & 8.33 & \\
\hline CXOMP J005622.8-272746 .................... & 2242 & 3 & 3 & 14.095076 & -27.462953 & 13.97 & 13.35 & 23.71 & 8.35 & \\
\hline CXOMP J005630.6-273141 ……............. & 2242 & 2 & 4 & 14.127555 & -27.528123 & 12.27 & 14.01 & 26.06 & 9.67 & 66 \\
\hline CXOMP J005645.6-272444 …................ & 2242 & 3 & 1 & 14.190344 & -27.412292 & 3.66 & 7.52 & 8.29 & 9.59 & \\
\hline CXOMP J005645.9-275522 …................ & 2243 & 3 & 4 & 14.191467 & -27.922784 & 8.72 & 14.68 & 28.46 & 7.53 & \\
\hline CXOMP J005649.8-273319 …............... & 2242 & 2 & 3 & 14.207559 & -27.555305 & 11.77 & 12.57 & 20.92 & 9.28 & \\
\hline 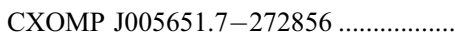 & 2242 & 2 & 1 & 14.215693 & -27.482293 & 2.70 & 8.74 & 10.92 & 10.86 & \\
\hline CXOMP J005654.5-275116 …................. & 2243 & 3 & 3 & 14.227221 & -27.854715 & 8.08 & 10.39 & 14.74 & 8.23 & \\
\hline CXOMP J005657.0-274025 …................. & 2244 & 3 & 1 & 14.237721 & -27.673876 & 4.54 & 11.58 & 18.08 & 8.04 & \\
\hline 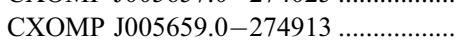 & 2243 & 3 & 2 & 14.246074 & -27.820540 & 6.08 & 8.27 & 9.92 & 8.59 & \\
\hline CXOMP J005705.3-274554 …................ & 2243 & 7 & 7 & 14.272302 & -27.765143 & 3.78 & 5.38 & 4.90 & 5.83 & \\
\hline CXOMP J005710.1-274952 …................ & 2246 & 3 & 2 & 14.292476 & -27.831169 & 6.09 & 12.02 & 19.31 & 8.42 & \\
\hline CXOMP J005710.2-274954 ................... & 2243 & 3 & 1 & 14.292750 & -27.831766 & 2.15 & 7.08 & 7.58 & 8.20 & 52 \\
\hline CXOMP J005712.9-272650 …............... & 2242 & 6 & 15 & 14.303934 & -27.447320 & 2.70 & 4.58 & 3.91 & 10.66 & \\
\hline CXOMP J005714.0-272536 .................... & 2242 & 6 & 4 & 14.308480 & -27.426900 & 2.22 & 3.33 & 3.00 & 10.88 & \\
\hline CXOMP J005714.9-271851 …................. & 2242 & 7 & 4 & 14.312284 & -27.314413 & 1.20 & 3.59 & 3.02 & 6.57 & \\
\hline CXOMP J005715.3-271735 …................. & 2242 & 7 & 7 & 14.313933 & -27.293127 & 2.20 & 4.84 & 4.15 & 6.10 & \\
\hline 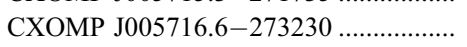 & 2244 & 7 & 11 & 14.319131 & -27.541602 & 1.18 & 5.03 & 4.35 & 6.47 & \\
\hline CXOMP J005716.8-272128 …................ & 2242 & 7 & 1 & 14.320197 & -27.357975 & 0.41 & 0.95 & 3.00 & 6.35 & 53 \\
\hline CXOMP J005717.3-274236 .................... & 2243 & 7 & 5 & 14.322400 & -27.710021 & 0.61 & 2.78 & 3.00 & 8.42 & \\
\hline CXOMP J005717.5-272617 …................. & 2242 & 6 & 7 & 14.322936 & -27.438214 & 1.42 & 3.90 & 3.30 & 10.74 & \\
\hline CXOMP J005717.9-271830 …................ & 2242 & 7 & 3 & 14.324672 & -27.308354 & 1.16 & 3.90 & 3.30 & 6.55 & 55 \\
\hline CXOMP J005719.7-272035 …................ & 2242 & 7 & 2 & 14.332301 & -27.343318 & 1.29 & 1.85 & 3.00 & 6.35 & \\
\hline
\end{tabular}

Notes.-Col. (1): ChaMP source name given by right ascension in units of hours, minutes, and seconds, and declination in units of degrees, arcminutes, and arcseconds in J2000.0. Col. (2): Observation ID. Col. (3): CCD ID (0-3 for ACIS-I and 4-9 for ACIS-S observation). Col. (4): Source number assigned by wavdetect as marked in the public X-ray image of the ChaMP Web site (http://hea-www.harvard.edu/CHAMP). Col. (5) and (6): Right ascension and declination in units of degrees after refinement and aspect correction. Col. (7): Positional uncertainty in units of arcseconds estimated by the $95 \%$ confidence level of the empirical formula (see eq. [12] in $\S 4.2$ ). Col. (8): Off-axis angle from the aim point of the observation in units of arcminutes. Col. (9): Source extraction radius in units of arcseconds (the $95 \%$ energy encircled radius at $1.5 \mathrm{keV}$. See $\S$ 3.2.1). Col. (10): Mean effective exposure time in units of ks after vignetting correction (see § 3.2.4). Col. (11): Source flag (see Table 4). Table 6 is available in its entirety in the electronic edition of the Astrophysical Journal Supplement. A portion is shown here for guidance regarding its form and content. 
TABLE 7

The Multiply Observed ChaMP X-Ray Point Source Candidates

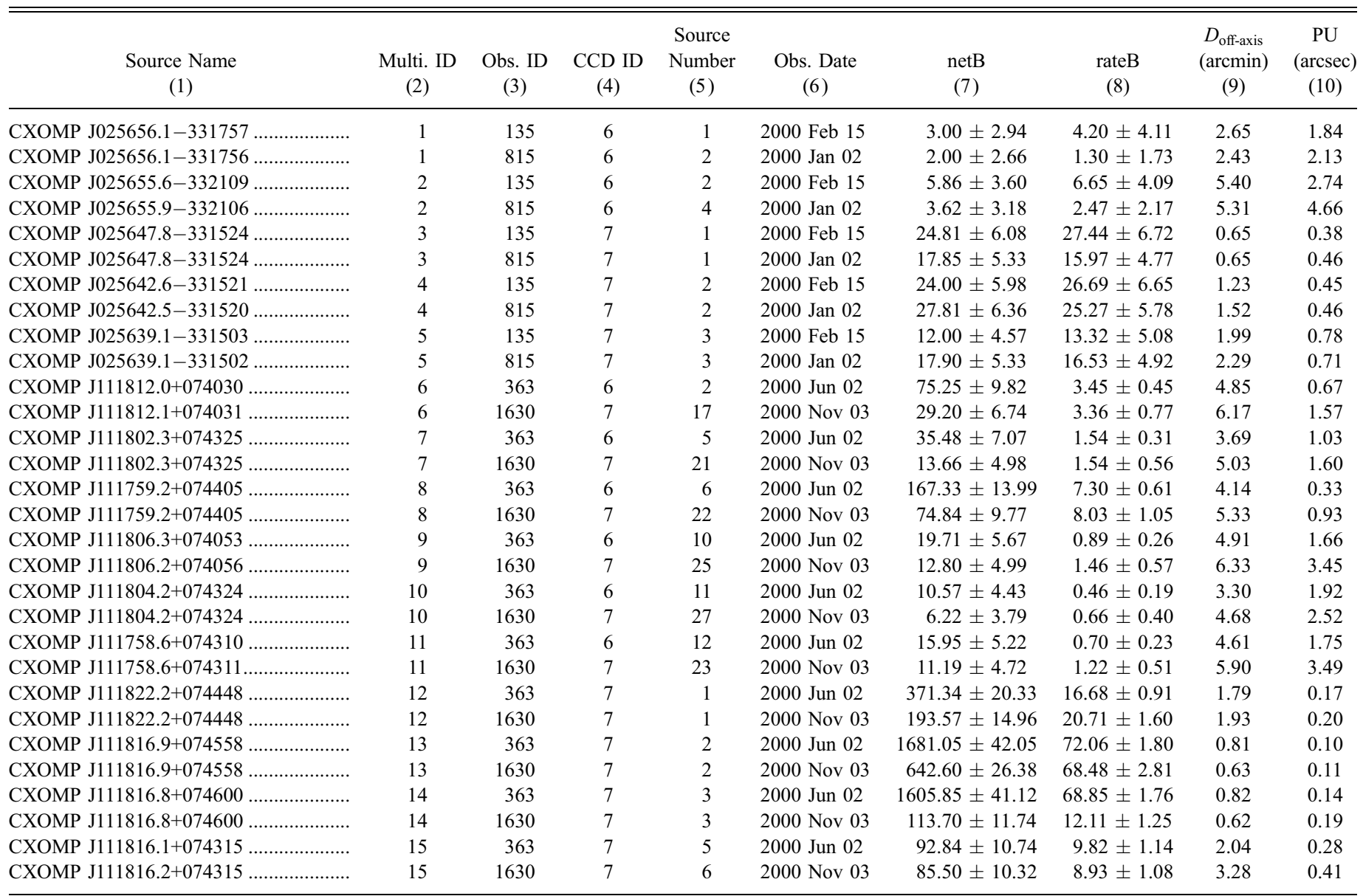

Notes.-Col. (1): ChaMP source name given by right ascension in units of hours, minutes, and seconds, and. declination in units of degrees, arcminutes, and arcseconds in J2000.0. Col. (2): Multiple observation ID indicates the same source candidate in overlapping fields, labeled with the same ID. Col. (3): Observation ID. Col. (4): CCD ID (0-3 for ACIS-I and 4-9 for ACIS-S). Col. (5): Source number assigned by wavdetect as marked in the public X-ray images of the ChaMP Web site (http://hea-www.harvard.edu/ CHAMP). Col. (6): Date of observation. Col. (7): Net counts in the 0.3-8 keV band and errors. Col. (8): Count rates in the 0.3-8 keV band and errors. Col. (9): off-axis angle from the aim point of the observation in units of arcminutes. Col. (10): Positional uncertainty in units of arcseconds estimated by the $95 \%$ confidence level of the empirical formula (see eq. [12] in $\S 4.2$ ). The errors in net counts are given following Gehrels statistics (Gehrels 1986). Table 7 is available in its entirety in the electronic edition of the Astrophysical Journal Supplement. A portion is shown here for guidance regarding its form and content. 
TABLE 8

The Main ChaMP X-Ray Point Source Photometry

\begin{tabular}{|c|c|c|c|c|c|c|c|c|}
\hline $\begin{array}{l}\text { Source Name } \\
\text { (1) }\end{array}$ & $\begin{array}{l}\text { B } \\
(2)\end{array}$ & $\begin{array}{l}S_{1} \\
(3)\end{array}$ & $\begin{array}{l}\mathrm{S}_{2} \\
\text { (4) }\end{array}$ & $\begin{array}{c}S \\
(5)\end{array}$ & $\begin{array}{l}\mathrm{H} \\
(6)\end{array}$ & $\begin{array}{l}\mathrm{Bc} \\
(7)\end{array}$ & $\begin{array}{l}\mathrm{Sc} \\
(8)\end{array}$ & $\begin{array}{l}\mathrm{Hc} \\
(9)\end{array}$ \\
\hline CXOMP J001709.7+162604 .. & $10.20 \pm 17.71$ & $5.55 \pm 9.03$ & $14.43 \pm 10.48$ & $19.98 \pm 13.40$ & & $6.13 \pm 17.38$ & $17.75 \pm 11.51$ & \\
\hline CXOMP J001716.0+162722 ……...................... & $94.40 \pm 20.17$ & $25.10 \pm 9.91$ & $48.11 \pm 12.09$ & $73.21 \pm 15.20$ & $21.19 \pm 13.91$ & $90.00 \pm 20.10$ & $60.42 \pm 13.42$ & $29.58 \pm 14.97$ \\
\hline CXOMP J001739.7+162330 …………….......... & $233.49 \pm 20.14$ & $51.89 \pm 9.68$ & $131.11 \pm 14.06$ & $183.00 \pm 16.66$ & $50.48 \pm 12.03$ & $234.00 \pm 20.48$ & $169.84 \pm 15.74$ & $64.16 \pm 13.11$ \\
\hline CXOMP J001743.5+163144 …………….......... & $20.29 \pm 9.20$ & & $16.27 \pm 6.38$ & $15.86 \pm 7.24$ & $4.43 \pm 6.39$ & $21.71 \pm 9.45$ & $18.72 \pm 6.76$ & $3.00 \pm 6.61$ \\
\hline CXOMP J001756.6+163006 …………….......... & $34.54 \pm 8.17$ & $6.26 \pm 4.14$ & $19.36 \pm 6.00$ & $25.62 \pm 6.84$ & $8.92 \pm 5.26$ & $30.65 \pm 8.28$ & $20.31 \pm 6.10$ & $10.34 \pm 5.60$ \\
\hline CXOMP J001758.4+162647 ………….............. & $29.16 \pm 8.14$ & $4.83 \pm 3.99$ & $14.49 \pm 5.59$ & $19.32 \pm 6.41$ & $9.85 \pm 5.74$ & $28.73 \pm 8.53$ & $20.11 \pm 6.21$ & $8.63 \pm 5.85$ \\
\hline CXOMP J001758.9+163119 …....................... & $58.38 \pm 9.57$ & $4.80 \pm 3.99$ & $41.65 \pm 7.78$ & $46.45 \pm 8.36$ & $11.93 \pm 5.48$ & $56.72 \pm 9.83$ & $43.87 \pm 8.00$ & $12.85 \pm 5.71$ \\
\hline CXOMP J001801.0+163139 ………….............. & $28.72 \pm 7.43$ & $3.82 \pm 3.62$ & $13.39 \pm 5.11$ & $17.21 \pm 5.79$ & $11.51 \pm 5.37$ & $27.78 \pm 7.74$ & $15.95 \pm 5.46$ & $11.83 \pm 5.48$ \\
\hline CXOMP J001801.4+163237 ………………...... & $22.07 \pm 6.94$ & & $7.49 \pm 4.45$ & $7.16 \pm 4.74$ & $14.91 \pm 5.70$ & $22.85 \pm 7.39$ & $4.71 \pm 4.15$ & $18.13 \pm 6.11$ \\
\hline CXOMP J001801.7+163426 ………….............. & $82.88 \pm 11.03$ & $3.16 \pm 3.62$ & $45.22 \pm 8.22$ & $48.39 \pm 8.63$ & $34.50 \pm 7.57$ & $82.30 \pm 11.38$ & $40.27 \pm 7.86$ & $42.03 \pm 8.23$ \\
\hline CXOMP J001803.4+162751 ……………......... & $19.90 \pm 6.68$ & $6.00 \pm 3.97$ & $9.22 \pm 4.59$ & $15.22 \pm 5.58$ & $4.69 \pm 4.47$ & $18.23 \pm 6.98$ & $13.24 \pm 5.23$ & $4.99 \pm 4.61$ \\
\hline CXOMP J001805.7+162852 …........................ & $19.23 \pm 6.20$ & $1.26 \pm 2.96$ & $6.74 \pm 3.97$ & $8.00 \pm 4.45$ & $11.23 \pm 4.99$ & $19.67 \pm 6.67$ & $5.82 \pm 3.98$ & $13.84 \pm 5.35$ \\
\hline 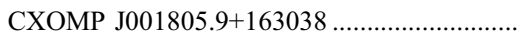 & $17.12 \pm 5.90$ & $4.67 \pm 3.61$ & $11.77 \pm 4.72$ & $16.44 \pm 5.46$ & $0.68 \pm 3.21$ & $17.45 \pm 6.35$ & $16.15 \pm 5.34$ & $1.30 \pm 3.43$ \\
\hline CXOMP J001806.7+162517 …………….......... & $11.27 \pm 6.17$ & $0.89 \pm 3.23$ & $7.36 \pm 4.47$ & $8.25 \pm 5.03$ & $3.02 \pm 4.35$ & $11.72 \pm 6.66$ & $7.84 \pm 4.76$ & $3.88 \pm 4.65$ \\
\hline CXOMP J001807.2+163551 ………………...... & $38.82 \pm 8.18$ & $0.36 \pm 2.69$ & $25.97 \pm 6.48$ & $26.33 \pm 6.66$ & $12.49 \pm 5.49$ & $39.55 \pm 8.64$ & $22.97 \pm 6.19$ & $16.57 \pm 6.02$ \\
\hline CXOMP J001807.9+163120 …............................ & $101.33 \pm 11.39$ & $7.00 \pm 3.97$ & $61.62 \pm 8.99$ & $68.62 \pm 9.48$ & $32.71 \pm 7.08$ & $100.71 \pm 11.80$ & $55.33 \pm 8.61$ & $45.38 \pm 8.07$ \\
\hline CXOMP J001808.5+163231 ….......................... & $81.25 \pm 10.44$ & $12.67 \pm 4.85$ & $42.57 \pm 7.70$ & $55.24 \pm 8.68$ & $26.01 \pm 6.57$ & $78.86 \pm 10.73$ & $48.31 \pm 8.13$ & $30.55 \pm 7.00$ \\
\hline 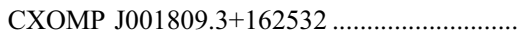 & $88.92 \pm 11.02$ & $15.70 \pm 5.35$ & $51.19 \pm 8.41$ & $66.90 \pm 9.55$ & $22.02 \pm 6.30$ & $86.98 \pm 11.35$ & $62.66 \pm 9.25$ & $24.32 \pm 6.58$ \\
\hline CXOMP J001809.8+162556 ………………........ & $25.35 \pm 6.77$ & $1.57 \pm 2.96$ & $20.07 \pm 5.79$ & $21.64 \pm 6.10$ & $3.71 \pm 3.83$ & $25.49 \pm 7.25$ & $13.35 \pm 5.12$ & $12.14 \pm 5.13$ \\
\hline CXOMP J001810.0+163208 ………………...... & $23.04 \pm 6.39$ & $0.82 \pm 2.67$ & $9.52 \pm 4.44$ & $10.34 \pm 4.73$ & $12.70 \pm 4.98$ & $22.70 \pm 6.77$ & $8.41 \pm 4.29$ & $14.29 \pm 5.23$ \\
\hline CXOMP J001810.2+162942 …….................... & $22.45 \pm 6.29$ & $7.41 \pm 3.96$ & $12.53 \pm 4.85$ & $19.95 \pm 5.78$ & $2.51 \pm 3.42$ & $22.60 \pm 6.74$ & $18.53 \pm 5.56$ & $4.07 \pm 3.81$ \\
\hline CXOMP J001810.2+163223 ………….............. & $269.67 \pm 17.61$ & $59.00 \pm 8.80$ & $156.73 \pm 13.60$ & $215.73 \pm 15.80$ & $53.94 \pm 8.61$ & $262.20 \pm 17.78$ & $189.63 \pm 14.85$ & $72.57 \pm 9.77$ \\
\hline CXOMP J001812.4+162713 ….......................... & $19.64 \pm 6.00$ & $1.79 \pm 2.95$ & $11.01 \pm 4.58$ & $12.79 \pm 4.99$ & $6.85 \pm 4.14$ & $19.69 \pm 6.49$ & $12.18 \pm 4.86$ & $7.51 \pm 4.30$ \\
\hline CXOMP J001817.6+163107 ………………....... & $34.42 \pm 7.07$ & $0.71 \pm 2.33$ & $19.76 \pm 5.56$ & $20.47 \pm 5.67$ & $13.95 \pm 4.98$ & $34.42 \pm 7.55$ & $16.57 \pm 5.22$ & $17.85 \pm 5.45$ \\
\hline CXOMP J001818.0+163316 …………….......... & $43.40 \pm 7.85$ & $3.58 \pm 3.19$ & $29.63 \pm 6.55$ & $33.20 \pm 6.90$ & $10.20 \pm 4.59$ & $43.56 \pm 8.30$ & $29.47 \pm 6.55$ & $14.09 \pm 5.11$ \\
\hline CXOMP J001818.8+163748 ............................... & $21.55 \pm 7.04$ & $3.89 \pm 3.62$ & $9.83 \pm 4.87$ & $13.72 \pm 5.60$ & $7.84 \pm 5.02$ & $22.37 \pm 7.51$ & $11.54 \pm 5.12$ & $10.84 \pm 5.50$ \\
\hline CXOMP J001820.0+162931 ……....................... & $11.15 \pm 4.72$ & $5.38 \pm 3.61$ & $2.48 \pm 2.94$ & $7.86 \pm 4.13$ & $3.29 \pm 3.19$ & $10.25 \pm 5.09$ & $7.05 \pm 3.97$ & $3.19 \pm 3.19$ \\
\hline CXOMP J001820.7+162315 ……...................... & $12.97 \pm 5.92$ & $0.36 \pm 2.68$ & $9.16 \pm 4.59$ & $9.52 \pm 4.87$ & $3.46 \pm 4.16$ & $13.21 \pm 6.41$ & $8.29 \pm 4.60$ & $4.93 \pm 4.47$ \\
\hline CXOMP J001821.2+163238 ………………...... & $6.38 \pm 3.79$ & $\ldots$ & $3.81 \pm 3.18$ & $3.66 \pm 3.18$ & $2.71 \pm 2.94$ & $6.43 \pm 4.34$ & $2.76 \pm 2.94$ & $3.66 \pm 3.18$ \\
\hline CXOMP J001821.7+161941 ……....................... & $138.87 \pm 14.31$ & $27.88 \pm 6.93$ & $94.16 \pm 11.32$ & $122.04 \pm 12.87$ & $16.83 \pm 7.07$ & $135.98 \pm 14.51$ & $116.89 \pm 12.43$ & $19.09 \pm 7.48$ \\
\hline
\end{tabular}

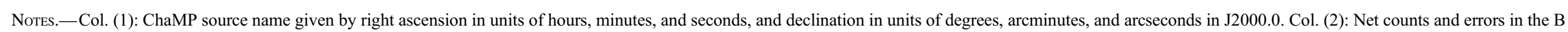

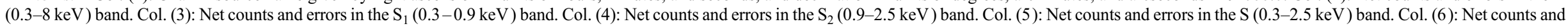

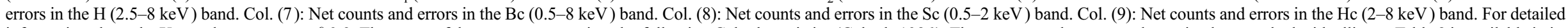

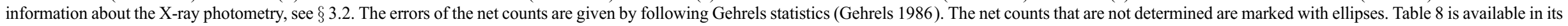
entirety in the electronic edition of the Astrophysical Journal Supplement. A portion is shown here for guidance regarding its form and content. 
TABLE 9

The Supplementary ChaMP X-Ray Point Source Photometry

\begin{tabular}{|c|c|c|c|c|c|c|c|c|}
\hline $\begin{array}{l}\text { Source Name } \\
\text { (1) }\end{array}$ & $\begin{array}{l}\mathrm{B} \\
(2)\end{array}$ & $\begin{array}{l}\mathrm{S}_{1} \\
\text { (3) }\end{array}$ & $\begin{array}{l}\mathrm{S}_{2} \\
\text { (4) }\end{array}$ & $\begin{array}{c}S \\
(5)\end{array}$ & $\begin{array}{c}\mathrm{H} \\
(6)\end{array}$ & $\begin{array}{l}\mathrm{Bc} \\
(7)\end{array}$ & $\begin{array}{l}\mathrm{Sc} \\
(8)\end{array}$ & $\begin{array}{l}\mathrm{Hc} \\
(9)\end{array}$ \\
\hline CXOMP J005618.9-272429 ……………........ & $16.06 \pm 6.51$ & $0.97 \pm 2.96$ & $12.10 \pm 4.99$ & $13.07 \pm 5.36$ & $2.99 \pm 4.48$ & $13.88 \pm 6.71$ & $7.72 \pm 4.45$ & $6.16 \pm 5.02$ \\
\hline CXOMP J005622.4-272223 ………………....... & $40.97 \pm 8.15$ & $12.45 \pm 4.85$ & $24.58 \pm 6.28$ & $37.03 \pm 7.48$ & $3.94 \pm 4.16$ & $36.65 \pm 8.23$ & $30.40 \pm 6.82$ & $6.25 \pm 4.61$ \\
\hline 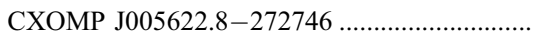 & $14.02 \pm 6.43$ & $3.71 \pm 3.62$ & $5.99 \pm 4.31$ & $9.70 \pm 5.14$ & $4.32 \pm 4.63$ & $13.42 \pm 6.82$ & $7.13 \pm 4.61$ & $6.29 \pm 5.03$ \\
\hline CXOMP J005630.6-273141 ……………........... & $32.02 \pm 8.48$ & $8.35 \pm 4.74$ & $18.61 \pm 6.11$ & $26.96 \pm 7.27$ & $5.07 \pm 5.16$ & $27.21 \pm 8.52$ & $20.34 \pm 6.50$ & $6.87 \pm 5.51$ \\
\hline CXOMP J005633.8-272954 …………............... & $59.47 \pm 9.69$ & $12.47 \pm 4.99$ & $36.54 \pm 7.48$ & $49.01 \pm 8.56$ & $10.46 \pm 5.37$ & $57.77 \pm 10.00$ & $46.50 \pm 8.28$ & $11.27 \pm 5.60$ \\
\hline CXOMP J005644.3-274936 ……....................... & $14.48 \pm 5.91$ & $0.94 \pm 2.67$ & $9.03 \pm 4.44$ & $9.97 \pm 4.73$ & $4.51 \pm 4.32$ & $14.06 \pm 6.30$ & $9.27 \pm 4.44$ & $4.79 \pm 4.47$ \\
\hline CXOMP J005645.6-272444 ………………....... & $10.36 \pm 4.58$ & $1.69 \pm 2.66$ & $5.59 \pm 3.60$ & $7.28 \pm 3.97$ & $3.08 \pm 3.19$ & $10.36 \pm 5.09$ & $7.33 \pm 3.97$ & $3.02 \pm 3.19$ \\
\hline CXOMP J005645.9-275522 ………………....... & $85.54 \pm 12.10$ & $22.51 \pm 6.40$ & $49.65 \pm 8.77$ & $72.16 \pm 10.43$ & $13.38 \pm 6.92$ & $83.65 \pm 12.35$ & $64.45 \pm 9.75$ & $19.20 \pm 7.58$ \\
\hline CXOMP J005649.8-273319 ............................... & $17.78 \pm 6.50$ & $6.47 \pm 4.14$ & $9.74 \pm 4.73$ & $16.21 \pm 5.80$ & $1.57 \pm 3.82$ & $18.91 \pm 6.96$ & $16.24 \pm 5.57$ & $2.66 \pm 4.16$ \\
\hline CXOMP J005651.7-272856 ................................. & $36.83 \pm 7.48$ & $0.47 \pm 2.33$ & $9.79 \pm 4.44$ & $10.25 \pm 4.58$ & $26.57 \pm 6.47$ & $36.98 \pm 7.90$ & $7.74 \pm 4.13$ & $29.24 \pm 6.74$ \\
\hline CXOMP J005654.5-275116 …………….......... & $10.10 \pm 5.49$ & $1.26 \pm 2.96$ & $6.44 \pm 4.14$ & $7.69 \pm 4.60$ & $2.40 \pm 3.82$ & $9.72 \pm 5.87$ & $8.10 \pm 4.45$ & $1.63 \pm 3.83$ \\
\hline CXOMP J005657.0-274025 ............................... & $45.14 \pm 9.17$ & $5.42 \pm 4.15$ & $31.94 \pm 7.25$ & $37.36 \pm 7.94$ & $7.78 \pm 5.38$ & $45.96 \pm 9.55$ & $33.75 \pm 7.41$ & $12.21 \pm 6.03$ \\
\hline CXOMP J005659.0-274913 ……………........... & $10.24 \pm 4.73$ & $0.61 \pm 2.33$ & $4.96 \pm 3.61$ & $5.57 \pm 3.80$ & $4.67 \pm 3.61$ & $9.39 \pm 5.11$ & $5.21 \pm 3.61$ & $4.18 \pm 3.62$ \\
\hline CXOMP J005659.3-272251 …............................ & $10.57 \pm 4.43$ & $4.89 \pm 3.40$ & $2.84 \pm 2.94$ & $7.73 \pm 3.96$ & $2.84 \pm 2.94$ & $7.62 \pm 4.50$ & $2.84 \pm 2.94$ & $4.78 \pm 3.40$ \\
\hline CXOMP J005701.0-272334 ………................... & $4.47 \pm 3.41$ & $\ldots$ & $2.84 \pm 2.94$ & $2.63 \pm 2.94$ & $1.84 \pm 2.66$ & $4.57 \pm 3.97$ & $2.84 \pm 2.94$ & $1.73 \pm 2.66$ \\
\hline CXOMP J005701.4-274344 ………………........ & $7.77 \pm 6.99$ & $0.01 \pm 3.44$ & & & $9.51 \pm 6.04$ & $8.43 \pm 7.34$ & & $8.51 \pm 6.15$ \\
\hline CXOMP J005704.7-280145 ............................... & $83.77 \pm 11.30$ & $15.47 \pm 5.47$ & $47.30 \pm 8.36$ & $62.77 \pm 9.57$ & $21.00 \pm 6.79$ & $79.52 \pm 11.49$ & $57.01 \pm 9.08$ & $22.51 \pm 7.05$ \\
\hline CXOMP J005705.3-274554 ………………....... & $6.93 \pm 5.15$ & $0.40 \pm 2.68$ & $6.46 \pm 4.31$ & $6.87 \pm 4.61$ & $0.06 \pm 3.22$ & $7.56 \pm 5.64$ & $6.52 \pm 4.31$ & $1.04 \pm 3.65$ \\
\hline CXOMP J005710.1-274952 …............................. & $40.96 \pm 8.09$ & $9.60 \pm 4.44$ & $32.81 \pm 6.99$ & $42.40 \pm 7.85$ & ... & $41.69 \pm 8.47$ & $39.86 \pm 7.55$ & $1.83 \pm 3.83$ \\
\hline CXOMP J005710.2-274954 _.............................. & $24.52 \pm 6.18$ & $1.86 \pm 2.66$ & $16.65 \pm 5.22$ & $18.51 \pm 5.45$ & $6.01 \pm 3.80$ & $24.52 \pm 6.66$ & $16.72 \pm 5.22$ & $7.80 \pm 4.14$ \\
\hline CXOMP J005712.9-272650 ………………........ & $4.43 \pm 3.41$ & $\ldots$ & $3.91 \pm 3.18$ & $3.76 \pm 3.18$ & $0.67 \pm 2.33$ & $4.43 \pm 3.97$ & $1.81 \pm 2.66$ & $2.62 \pm 2.94$ \\
\hline CXOMP J005714.0-272536 ............................... & $2.52 \pm 2.94$ & $1.00 \pm 2.33$ & $1.86 \pm 2.66$ & $2.86 \pm 2.94$ & & $1.52 \pm 3.29$ & $0.86 \pm 2.33$ & $0.67 \pm 2.33$ \\
\hline 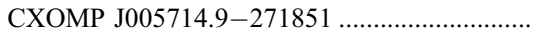 & $15.62 \pm 5.10$ & $8.86 \pm 4.12$ & $4.95 \pm 3.40$ & $13.81 \pm 4.84$ & $1.81 \pm 2.66$ & $15.67 \pm 5.55$ & $12.86 \pm 4.71$ & $2.81 \pm 2.94$ \\
\hline 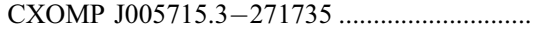 & $9.21 \pm 4.29$ & $0.67 \pm 2.33$ & $7.86 \pm 3.96$ & $8.53 \pm 4.13$ & $0.67 \pm 2.33$ & $9.35 \pm 4.74$ & $8.72 \pm 4.13$ & $0.63 \pm 2.33$ \\
\hline CXOMP J005716.6-273230 ……………............ & $33.84 \pm 8.46$ & $6.46 \pm 4.14$ & $17.39 \pm 5.90$ & $23.85 \pm 6.76$ & $9.99 \pm 5.83$ & $32.67 \pm 8.79$ & $19.95 \pm 6.20$ & $12.72 \pm 6.23$ \\
\hline CXOMP J005716.8-272128 .............................. & $24.57 \pm 6.08$ & $4.95 \pm 3.40$ & $14.86 \pm 4.97$ & $19.81 \pm 5.56$ & $4.76 \pm 3.40$ & $22.62 \pm 6.32$ & $17.90 \pm 5.33$ & $4.71 \pm 3.40$ \\
\hline CXOMP J005717.3-274236 ……....................... & $29.94 \pm 7.34$ & $8.08 \pm 4.13$ & $17.73 \pm 5.57$ & $25.82 \pm 6.47$ & $4.12 \pm 4.32$ & $28.27 \pm 7.63$ & $23.78 \pm 6.18$ & $4.49 \pm 4.47$ \\
\hline CXOMP J005717.5-272617 ………………......... & $19.71 \pm 5.56$ & $4.95 \pm 3.40$ & $5.95 \pm 3.60$ & $10.90 \pm 4.43$ & $8.81 \pm 4.12$ & $17.71 \pm 5.82$ & $5.91 \pm 3.60$ & $11.81 \pm 4.57$ \\
\hline 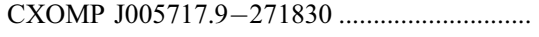 & $34.71 \pm 6.98$ & $17.95 \pm 5.33$ & $11.95 \pm 4.57$ & $29.90 \pm 6.55$ & $4.81 \pm 3.40$ & $24.71 \pm 6.53$ & $18.90 \pm 5.45$ & $5.81 \pm 3.60$ \\
\hline CXOMP J005719.7-272035 ……………........... & $4.48 \pm 3.40$ & $2.81 \pm 2.94$ & $0.95 \pm 2.33$ & $3.76 \pm 3.18$ & $0.71 \pm 2.33$ & $3.52 \pm 3.75$ & $2.81 \pm 2.94$ & $0.71 \pm 2.33$ \\
\hline
\end{tabular}

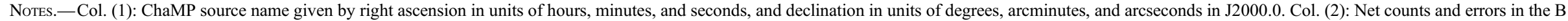

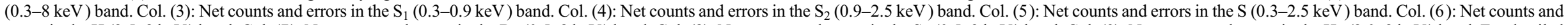

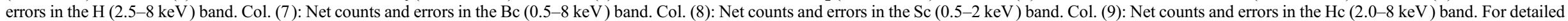

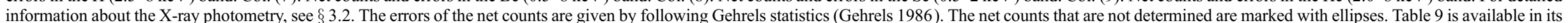
entirety in the electronic edition of the Astrophysical Journal Supplement. A portion is shown here for guidance regarding its form and content. 
TABLE 10

The Main ChaMP X-Ray Point Source Hardness Ratio and X-Ray Colors

\begin{tabular}{|c|c|c|c|c|c|c|c|c|c|}
\hline $\begin{array}{c}\text { Source Name } \\
\text { (1) }\end{array}$ & $\begin{array}{l}\text { HR_168 } \\
\text { (2) }\end{array}$ & $\begin{array}{c}\text { HR_mean } \\
\text { (3) }\end{array}$ & $\begin{array}{l}\text { HR_u68 } \\
\text { (4) }\end{array}$ & $\begin{array}{c}\mathrm{C} 21_{-} 168 \\
(5)\end{array}$ & $\begin{array}{c}\mathrm{C} 21 \text { _mode } \\
\text { (6) }\end{array}$ & $\begin{array}{c}\text { C21_u68 } \\
(7)\end{array}$ & $\begin{array}{c}\text { C32_168 } \\
(8)\end{array}$ & $\begin{array}{c}\text { C32_mode } \\
\text { (9) }\end{array}$ & $\begin{array}{c}\text { C32_u68 } \\
\text { (10) }\end{array}$ \\
\hline CXOMP J001709.7+162604 „.............. & -1.00 & -0.46 & -0.32 & -0.70 & -0.22 & 0.20 & -0.10 & 0.36 & 0.96 \\
\hline CXOMP J001716.0+162722.. & -0.53 & -0.35 & -0.10 & -0.45 & -0.28 & -0.08 & 0.02 & 0.23 & 0.53 \\
\hline CXOMP J001739.7+162330 .............. & -0.53 & -0.45 & -0.37 & -0.48 & -0.39 & -0.32 & 0.30 & 0.37 & 0.51 \\
\hline CXOMP J001743.5+163144.. & -0.90 & -0.54 & -0.33 & -1.22 & -0.70 & -0.36 & 0.04 & 0.34 & 0.74 \\
\hline CXOMP J001756.6+163006 ............... & -0.53 & -0.31 & -0.10 & -0.66 & -0.44 & -0.22 & 0.08 & 0.28 & 0.52 \\
\hline CXOMP J001758.4+162647 .............. & -0.62 & -0.38 & -0.14 & -0.68 & -0.40 & -0.14 & -0.08 & 0.14 & 0.38 \\
\hline CXOMP J001758.9+163119 ............. & -0.67 & -0.53 & -0.40 & -1.10 & -0.84 & -0.62 & 0.35 & 0.50 & 0.68 \\
\hline CXOMP J001801.0+163139 .............. & -0.36 & -0.15 & 0.08 & -0.76 & -0.44 & -0.20 & -0.14 & 0.05 & 0.26 \\
\hline CXOMP J001801.4+163237 .............. & 0.35 & 0.54 & 0.78 & -1.20 & -0.66 & -0.26 & -0.50 & -0.26 & -0.06 \\
\hline CXOMP J001801.7+163426 ............. & -0.09 & 0.02 & 0.14 & -1.32 & -1.00 & -0.76 & 0.01 & 0.10 & 0.22 \\
\hline CXOMP J001803.4+162751 .............. & -0.70 & -0.41 & -0.14 & -0.42 & -0.16 & 0.08 & -0.08 & 0.20 & 0.54 \\
\hline CXOMP J001805.7+162852 .............. & 0.17 & 0.37 & 0.62 & -0.90 & -0.44 & -0.10 & -0.42 & -0.20 & 0.02 \\
\hline CXOMP J001805.9+163038 ...... & -0.99 & -0.71 & -0.63 & -0.60 & -0.34 & -0.12 & 0.32 & 0.66 & 1.14 \\
\hline CXOMP J001806.7+162517 .............. & -0.66 & -0.26 & 0.10 & -0.96 & -0.46 & -0.10 & -0.12 & 0.22 & 0.64 \\
\hline CXOMP J001807.2+163551 ............. & -0.33 & -0.16 & 0.03 & -1.60 & -1.10 & -0.78 & 0.13 & 0.29 & 0.47 \\
\hline CXOMP J001807.9+163120 ............. & -0.20 & -0.10 & 0.00 & -1.08 & -0.88 & -0.74 & 0.17 & 0.27 & 0.37 \\
\hline CXOMP J001808.5+163231 .............. & -0.35 & -0.22 & -0.12 & -0.65 & -0.50 & -0.37 & 0.09 & 0.19 & 0.32 \\
\hline CXOMP J001809.3+162532 .............. & -0.54 & -0.43 & -0.33 & -0.63 & -0.48 & -0.37 & 0.23 & 0.36 & 0.47 \\
\hline CXOMP J001809.8+162556 .............. & -0.26 & -0.05 & 0.17 & -1.24 & -0.84 & -0.56 & 0.36 & 0.60 & 0.92 \\
\hline CXOMP J001810.0+163208 „............. & 0.04 & 0.24 & 0.46 & -1.12 & -0.66 & -0.32 & -0.30 & -0.12 & 0.08 \\
\hline CXOMP J001810.2+162942 ............. & -0.81 & -0.59 & -0.42 & -0.42 & -0.20 & -0.02 & 0.24 & 0.52 & 0.90 \\
\hline CXOMP J001810.2+163223 ............. & -0.50 & -0.44 & -0.39 & -0.49 & -0.42 & -0.35 & 0.39 & 0.46 & 0.53 \\
\hline CXOMP J001812.4+162713 „.............. & -0.46 & -0.22 & 0.01 & -0.96 & -0.58 & -0.28 & -0.04 & 0.18 & 0.40 \\
\hline CXOMP J001817.6+163107 .............. & -0.14 & 0.04 & 0.20 & -1.48 & -1.06 & -0.72 & -0.01 & 0.14 & 0.29 \\
\hline CXOMP J001818.0+163316 .............. & -0.49 & -0.34 & -0.20 & -1.06 & -0.82 & -0.60 & 0.28 & 0.43 & 0.60 \\
\hline CXOMP J001818.8+163748 „............. & -0.28 & -0.04 & 0.23 & -0.64 & -0.32 & -0.04 & -0.18 & 0.08 & 0.34 \\
\hline CXOMP J001820.0+162931 .............. & -0.64 & -0.32 & -0.06 & -0.06 & 0.26 & 0.58 & -0.46 & -0.08 & 0.26 \\
\hline CXOMP J001820.7+162315 „............. & -0.56 & -0.22 & 0.11 & -1.20 & -0.68 & -0.32 & -0.02 & 0.28 & 0.66 \\
\hline CXOMP J001821.2+163238 .............. & -0.23 & 0.11 & 0.49 & -1.28 & -0.68 & -0.22 & -0.20 & 0.10 & 0.44 \\
\hline CXOMP J001821.7+161941 .............. & -0.79 & -0.71 & -0.62 & -0.62 & -0.50 & -0.42 & 0.56 & 0.68 & 0.88 \\
\hline
\end{tabular}

Notes._Col. (1): ChaMP source name given by right ascension in units of hours, minutes, and seconds, and declination in units of degrees, arcminutes, and arcseconds in J2000.0. Col. (2): The $68 \%$ lower limit of hardness ratio HR. Col. (3): Mean of hardness ratio [HR $=(\mathrm{Hc}-\mathrm{Sc}) /(\mathrm{Hc}+\mathrm{Sc})]$. Col. (4): The $68 \%$ upper limit of hardness ratio HR. Col. (5): The 68\% lower limit of color C21. Col. (6): Mode of X-ray color C21 [ $\left.\log \left(\mathrm{S}_{1} / \mathrm{S}_{2}\right)\right]$. Col. (7): The 68\% upper limit of color C21. Col. (8): The 68\% lower limit of color C32. Col. (9): Mode of X-ray color C32 [ $\left.\log \left(\mathrm{S}_{2} / \mathrm{H}\right)\right]$. Col. (10): The $68 \%$ upper limit of color C32. For detailed information about photometry and

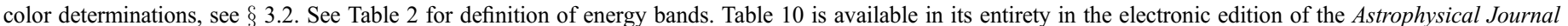
Supplement. A portion is shown here for guidance regarding its form and content. 
TABLE 11

The Supplementary ChaMP X-Ray Point Source Hardness Ratio and X-Ray Colors

\begin{tabular}{|c|c|c|c|c|c|c|c|c|c|}
\hline $\begin{array}{c}\text { Source Name } \\
\text { (1) }\end{array}$ & $\begin{array}{c}\text { HR_168 } \\
\text { (2) }\end{array}$ & $\begin{array}{c}\text { HR_mean } \\
\text { (3) }\end{array}$ & $\begin{array}{c}\text { HR_u68 } \\
\text { (4) }\end{array}$ & $\begin{array}{c}\mathrm{C} 21 \_168 \\
(5)\end{array}$ & $\begin{array}{c}\text { C21_mode } \\
(6)\end{array}$ & $\begin{array}{c}\mathrm{C} 21 \_\mathrm{u} 68 \\
(7)\end{array}$ & $\begin{array}{c}\text { C32_168 } \\
\text { (8) }\end{array}$ & $\begin{array}{c}\text { C32_mode } \\
\text { (9) }\end{array}$ & $\begin{array}{c}\text { C32_u68 } \\
\text { (10) }\end{array}$ \\
\hline CXOMP J005618.9-272429 ...... & -0.43 & -0.11 & 0.26 & -1.14 & -0.70 & -0.36 & 0.10 & 0.40 & 0.80 \\
\hline CXOMP J005622.4-272223 ................ & -0.80 & -0.63 & -0.48 & -0.44 & -0.28 & -0.13 & 0.40 & 0.64 & 0.98 \\
\hline CXOMP J005622.8-272746 ................ & -0.40 & -0.06 & 0.32 & -0.52 & -0.16 & 0.16 & -0.28 & 0.06 & 0.46 \\
\hline CXOMP J005630.6-273141 .................. & -0.71 & -0.46 & -0.22 & -0.54 & -0.30 & -0.10 & 0.16 & 0.42 & 0.76 \\
\hline CXOMP J005633.8-272954 ................. & -0.73 & -0.59 & -0.47 & -0.60 & -0.44 & -0.30 & 0.33 & 0.50 & 0.70 \\
\hline CXOMP J005644.3-274936 ................ & -0.60 & -0.29 & 0.04 & -1.08 & -0.64 & -0.28 & -0.08 & 0.22 & 0.54 \\
\hline CXOMP J005645.6-272444 .................. & -0.68 & -0.35 & -0.10 & -0.76 & -0.38 & -0.06 & -0.10 & 0.20 & 0.52 \\
\hline CXOMP J005645.9-275522 ................ & -0.66 & -0.53 & -0.40 & -0.46 & -0.33 & -0.22 & 0.34 & 0.51 & 0.72 \\
\hline CXOMP J005649.8-273319 ................ & -0.90 & -0.61 & -0.44 & -0.40 & -0.16 & 0.08 & 0.12 & 0.44 & 0.90 \\
\hline CXOMP J005651.7-272856 .................. & 0.43 & 0.55 & 0.71 & -1.26 & -0.80 & -0.42 & -0.58 & -0.40 & -0.26 \\
\hline CXOMP J005654.5-275116 .................. & -0.89 & -0.47 & -0.24 & -0.88 & -0.44 & -0.06 & -0.10 & 0.24 & 0.68 \\
\hline CXOMP J005657.0-274025 ............... & -0.63 & -0.46 & -0.29 & -0.94 & -0.68 & -0.48 & 0.32 & 0.54 & 0.78 \\
\hline CXOMP J005659.0-274913 ................. & -0.43 & -0.10 & 0.24 & -1.00 & -0.52 & -0.12 & -0.26 & 0.02 & 0.32 \\
\hline CXOMP J005659.3-272251 ................. & -0.08 & 0.20 & 0.56 & -0.10 & 0.18 & 0.50 & -0.34 & 0.00 & 0.34 \\
\hline CXOMP J005701.0-272334 ................ & -0.61 & -0.17 & 0.19 & -1.20 & -0.58 & -0.10 & -0.24 & 0.12 & 0.50 \\
\hline CXOMP J005701.4-274344 ................. & 0.40 & 0.51 & 1.00 & -0.54 & 0.08 & 0.74 & -1.28 & -0.70 & -0.26 \\
\hline CXOMP J005704.7-280145 ................ & -0.55 & -0.42 & -0.31 & -0.62 & -0.45 & -0.35 & 0.21 & 0.35 & 0.48 \\
\hline CXOMP J005705.3-274554 ................ & -0.93 & -0.44 & -0.23 & -1.08 & -0.56 & -0.16 & 0.08 & 0.48 & 1.02 \\
\hline CXOMP J005710.1-274952 ................ & -0.99 & -0.84 & -0.80 & -0.66 & -0.50 & -0.34 & 0.88 & 1.24 & 1.80 \\
\hline CXOMP J005710.2-274954 ................ & -0.54 & -0.34 & -0.16 & -1.10 & -0.78 & -0.52 & 0.20 & 0.40 & 0.60 \\
\hline CXOMP J005712.9-272650 ................ & -0.24 & 0.12 & 0.58 & -1.30 & -0.70 & -0.24 & 0.04 & 0.42 & 0.90 \\
\hline CXOMP J005714.0-272536 ................ & -0.62 & -0.04 & 0.50 & -0.62 & -0.16 & 0.26 & -0.06 & 0.46 & 1.08 \\
\hline CXOMP J005714.9-271851 ….............. & -0.81 & -0.57 & -0.43 & 0.00 & 0.22 & 0.46 & 0.00 & 0.32 & 0.68 \\
\hline CXOMP J005715.3-271735 ................. & -1.00 & -0.70 & -0.63 & -1.14 & -0.68 & -0.32 & 0.32 & 0.68 & 1.14 \\
\hline CXOMP J005716.6-273230 ................. & -0.43 & -0.22 & 0.01 & -0.62 & -0.38 & -0.18 & -0.02 & 0.20 & 0.44 \\
\hline CXOMP J005716.8-272128 ................ & -0.74 & -0.54 & -0.40 & -0.64 & -0.42 & -0.22 & 0.22 & 0.44 & 0.66 \\
\hline CXOMP J005717.3-274236 ................ & -0.85 & -0.63 & -0.47 & -0.50 & -0.32 & -0.14 & 0.24 & 0.50 & 0.84 \\
\hline CXOMP J005717.5-272617 ................. & 0.11 & 0.30 & 0.54 & -0.32 & -0.06 & 0.18 & -0.38 & -0.16 & 0.06 \\
\hline CXOMP J005717.9-271830 .................. & -0.68 & -0.49 & -0.35 & 0.01 & 0.17 & 0.32 & 0.14 & 0.34 & 0.58 \\
\hline CXOMP J005719.7-272035 ................ & -0.89 & -0.37 & -0.12 & -0.10 & 0.28 & 0.74 & -0.48 & 0.02 & 0.56 \\
\hline
\end{tabular}

Notes.-Col. (1): ChaMP source name given by right ascension in units of hours, minutes, and seconds, and declination in units of degrees, arcminutes, and arcseconds in J2000.0. Col. (2): The $68 \%$ lower limit of hardness ratio HR. Col. (3): Mean of hardness ratio [ HR $=(\mathrm{Hc}-\mathrm{Sc}) /(\mathrm{Hc}+\mathrm{Sc})]$. Col. (4): The $68 \%$ upper limit of hardness ratio HR.

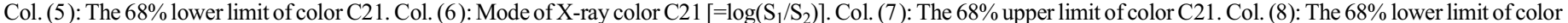

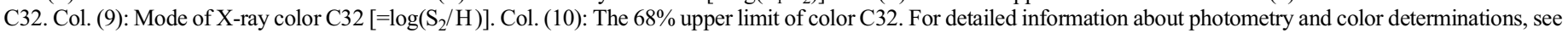

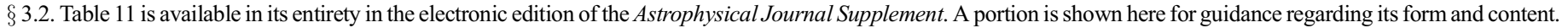

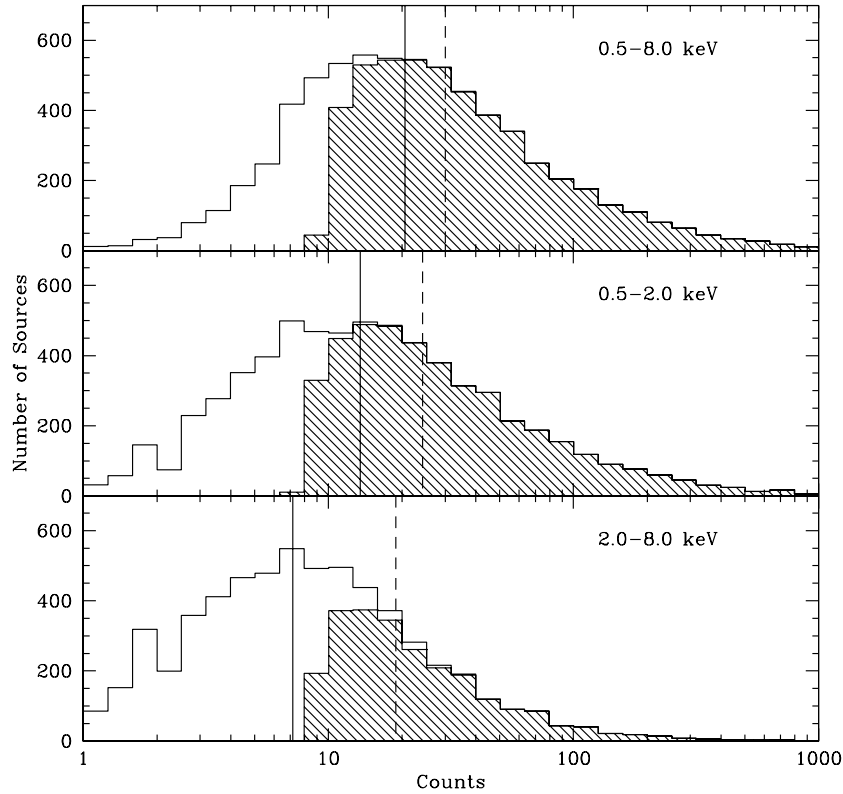

FIG. 16.-Distributions of source net counts in the $\mathrm{Bc}$ (top), Sc (middle), and $\mathrm{Hc}$ (bottom) bands, respectively. The open and shade histograms are for all sources and sources with $\mathrm{S} / \mathrm{N}>2.0$, respectively, in each energy band. The solid and dashed vertical lines indicate the medians of the total sample and high-S/N sample, respectively.

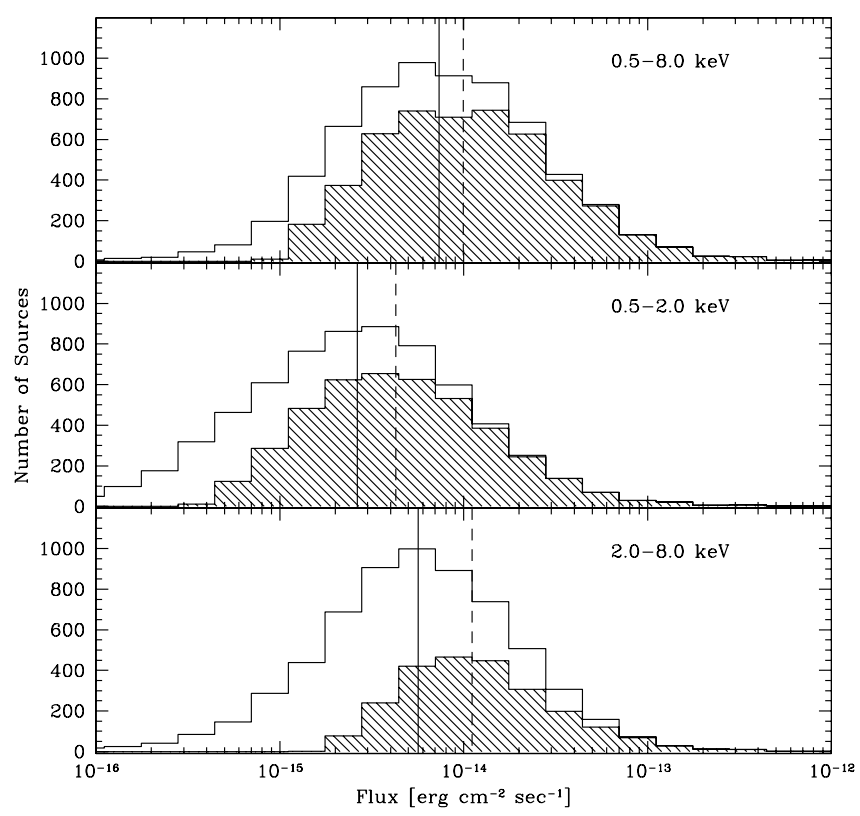

FIG. 17.- Same as Fig. 16, but for flux. The flux was determined assuming a photon index of $\Gamma_{\mathrm{ph}}=1.7$ and Galactic absorption $N_{\mathrm{H}}$. 


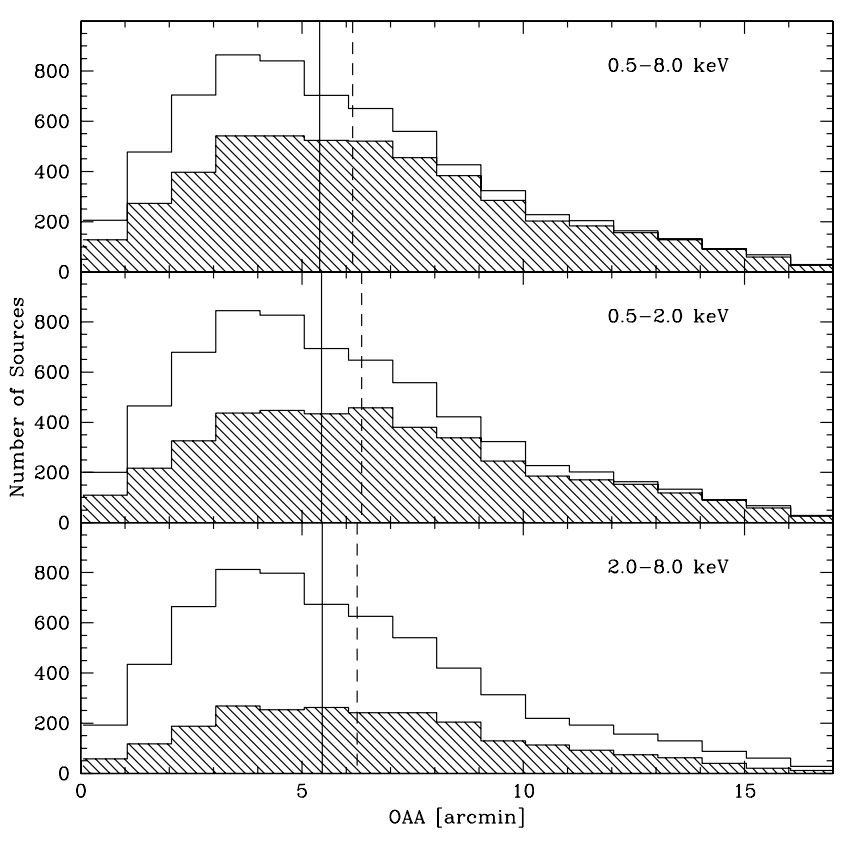

FIG. 18.- Same as Fig. 16, but for off-axis angle.

a 95\% confidence level positional uncertainty (see eq. [12] in $\S 4.2 .1)$. These sources also have a source flag of 52 (see $\S 3.2 .5$ ). Table 7 allows us to investigate the variability of X-ray source brightness.

The ChaMP source name is given by its right ascension and declination. We note that the position of some sources in Paper I and this paper could be slightly different because the position refinement process was applied to sources with an off-axis angle of $>400^{\prime \prime}$ in Paper I, whereas in this paper it was applied to all sources. For the sources that were published in previous papers (Kim et al. 2004a; Green et al. 2004; Silverman et al. 2005a, $2005 \mathrm{~b}$ ), we use the published name, even if the source position has been changed. The full versions of the tables are available in the electronic version of this paper and also on the ChaMP Web site. $^{22}$

\subsection{The ChaMP X-Ray Point Sources}

To understand the properties of the ChaMP X-ray point sources, we investigated their statistical characteristics. After eliminating source duplications in the main and supplementary ChaMP catalogs, the ChaMP X-ray point source catalog contains 6889 unique sources. To eliminate the source duplication, we selected a source having the smallest positional uncertainty among the same source candidates in Table 7: since the positional uncertainty is a function of off-axis angle and source counts (see $\S 4.2 .1$ ), this criterion automatically selects a higher quality source. With these individual sources, we display the distributions of source count, flux, off-axis angle, and hardness ratio and colors of these individual sources with their median values in Figures 16, 17, 18, and 19, respectively. Sources with signal-to-noise ratio $\mathrm{S} / \mathrm{N}>2.0$ are displayed with shaded histograms. Figures 20 and 21 show the $\mathrm{X}$-ray color-color diagrams of the ChaMP X-ray point sources in four CCD chips observed with ACIS-I and ACIS-S, respectively. The grid in the X-ray color-color diagram indicates the predicted locations of sources at redshift $z=0$ with various photon indices $\left(0 \leq \Gamma_{\mathrm{ph}} \leq 4\right)$ and neutral hydrogen column densities $\left(10^{20} \leq\right.$

\footnotetext{
${ }^{22}$ See http://hea-www.cfa.harvard.edu/CHAMP.
}
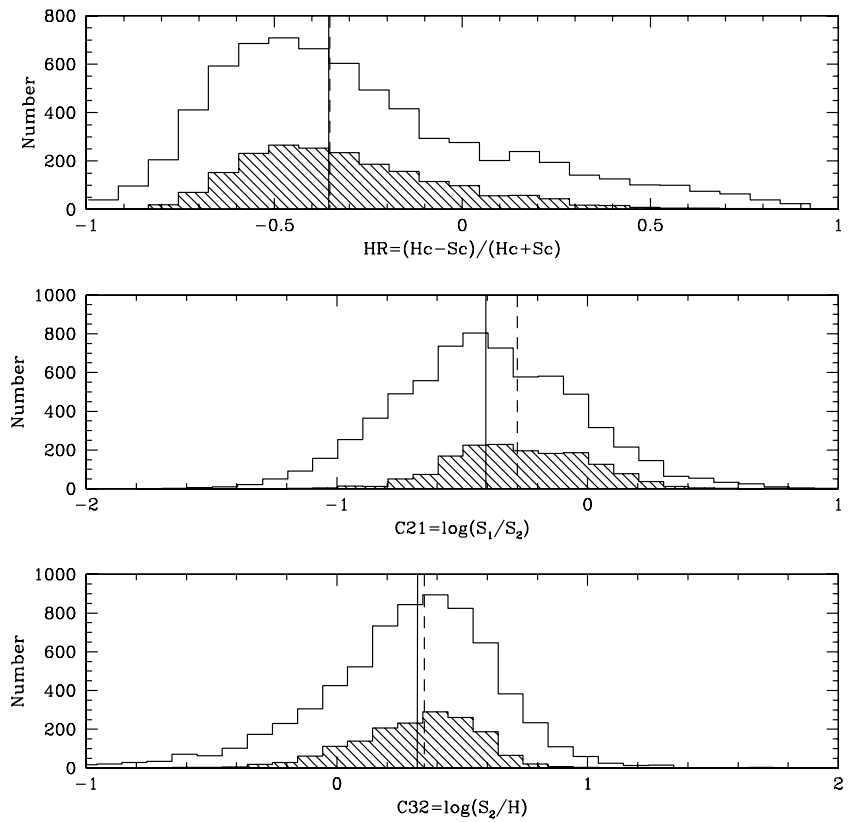

FIG. 19.-Number distribution of mean hardness ratio (top), the most probable values of the color C21 (middle) and C32 (bottom) of ChaMP X-ray point sources. The open and shaded histograms are for sources with $\mathrm{S} / \mathrm{N} \geq 0$ and $\mathrm{S} / \mathrm{N}>2$ in both energy bands, respectively. This constraint yields a small number of shaded histograms. The solid and dashed vertical lines indicate the medians of the total sample and high-S/N sample, respectively.

$N_{\mathrm{H}} \leq 10^{22}$ ). Sources with $\mathrm{S} / \mathrm{N}>1.5$ (open circles) and sources with $\mathrm{S} / \mathrm{N}>2.0$ (filled circles) are plotted. Most sources have absorption in the range $10^{20} \mathrm{~cm}^{-2} \lesssim N_{\mathrm{H}} \lesssim 10^{21} \mathrm{~cm}^{-2}$ and the photon index, $1 \lesssim \Gamma_{\mathrm{ph}} \lesssim 2.5$. We note that the absorbed sources $\left(N_{\mathrm{H}}>10^{21} \mathrm{~cm}^{-2}\right)$ in the ChaMP sample are not statistically significant $(\mathrm{S} / \mathrm{N}<1.5$ in at least one energy band) and so are not plotted in Figures 20 and 21.

In Table 12, we summarize the statistical properties of the ChaMP X-ray point sources: number of sources, minimum, maximum, median, and mean for the source counts, source fluxes, effective exposure times, off-axis angle, hardness ratio HR, color $\mathrm{C} 21$, and color $\mathrm{C} 32$. We define the properties of typical ChaMP $\mathrm{X}$-ray point source as the median values of these quantities for sources with $\mathrm{S} / \mathrm{N}>2.0$.

The typical source fluxes are $4.3 \times 10^{-15} \mathrm{erg} \mathrm{cm}^{-2} \mathrm{~s}^{-1}(0.5-$ $2 \mathrm{keV})$ and $11.1 \times 10^{-15} \mathrm{erg} \mathrm{cm}^{-2} \mathrm{~s}^{-1}(2-8 \mathrm{keV})$, respectively. The typical flux ranges are $3.7 \times 10^{-16}-2.5 \times 10^{-11} \mathrm{erg} \mathrm{cm}^{-2} \mathrm{~s}^{-1}$ $(0.5-2 \mathrm{keV})$ and $1.7 \times 10^{-15}-6.7 \times 10^{-11} \mathrm{erg} \mathrm{cm}^{-2} \mathrm{~s}^{-1}(2-$ $8 \mathrm{keV}$ ), respectively. The flux ranges of ChaMP X-ray sources cover the flux gap between the Chandra Deep Fields and previous surveys such as $A S C A$ and $R O S A T$, and fully cover the flux range around the break in the X-ray number counts (Kim et al. 2006b).

Thanks to the ChaMP's medium depth $\left(9.4 \times 10^{-16}-5.9 \times\right.$ $10^{-11} \mathrm{erg} \mathrm{cm}^{-2} \mathrm{~s}^{-1}$ in the $\left.0.5-8 \mathrm{keV}\right)$, wide sky coverage area $\left(\sim 10 \mathrm{deg}^{2}\right)$, and large number of sources $(\sim 6800)$, we can investigate populations and evolution models of cosmic X-ray sources with small statistical errors. The ChaMP is a serendipitous survey, therefore it is suitable for investigating the field-to-field variations of X-ray sources. In later ChaMP papers, we will provide number counts of X-ray point sources and their contributions to the CXRB, spatial angular correlations, X-ray color-color analysis, and optical/IR/radio properties of X-ray sources. Also, we will extend our study of X-ray galaxies (Kim et al. 2006a) and X-ray galaxy clusters (Barkhouse et al. 2006) in ChaMP fields. 

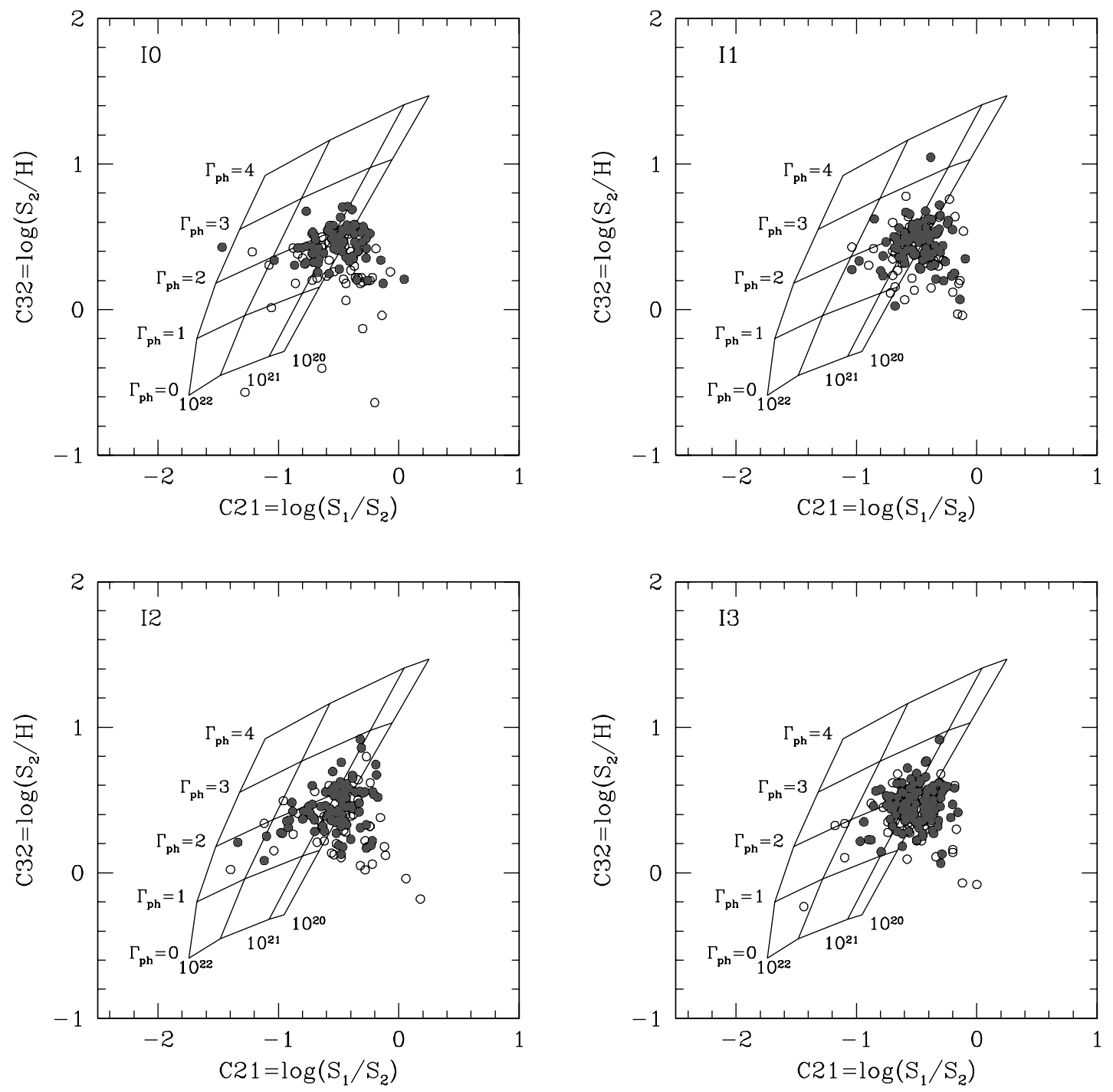

FIG. 20.-Color-color diagram of ChaMP X-ray point sources observed with ACIS-I. The open circles and filled circles represent sources with S/N $>1.5$ and with $\mathrm{S} / \mathrm{N}>2.0$, respectively. The grid indicates the predicted locations of the sources at redshift $z=0$ with various photon indices $\left(0 \leq \Gamma_{\mathrm{ph}} \leq 4\right.$, from bottom to top $)$ and absorption column densities $\left(10^{20} \mathrm{~cm}^{-2} \leq N_{\mathrm{H}} \leq 10^{22} \mathrm{~cm}^{-2}\right.$, from right to left). Most sources are located within the ranges of Galactic absorption $10^{20} \mathrm{~cm}^{-2} \leq N_{\mathrm{H}} \leq$ $10^{22} \mathrm{~cm}^{-2}$ and photon index $1 \lesssim \Gamma_{\mathrm{ph}} \lesssim 2.5$. [See the electronic edition of the Supplement for a color version of this figure.] 

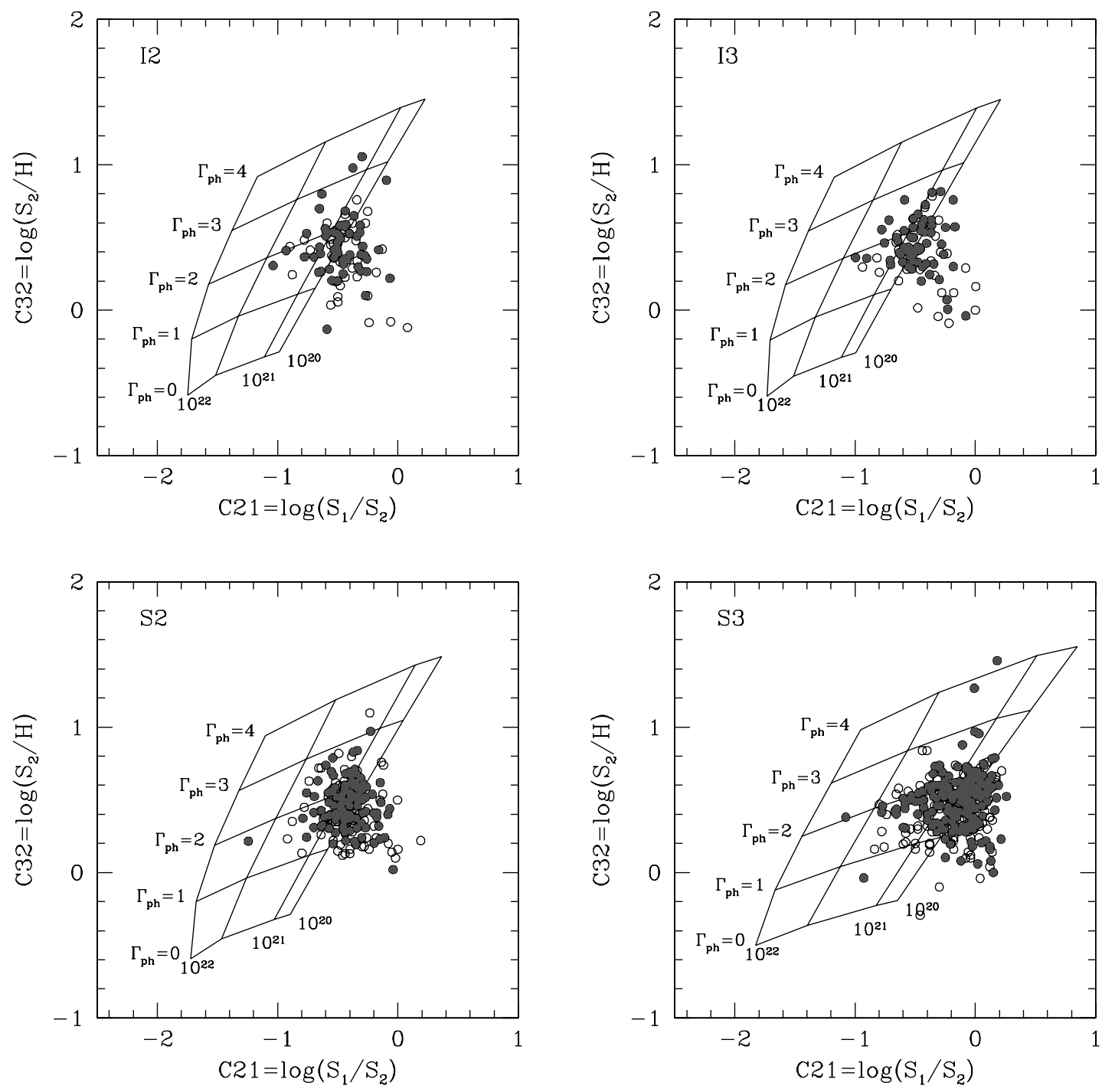

FIG. 21.-Same as Fig. 20, but for ACIS-S observations. [See the electronic edition of the Supplement for a color version of this figure.] 
TABLE 12

Statistical Summary of the ChaMP X-Ray Point Sources

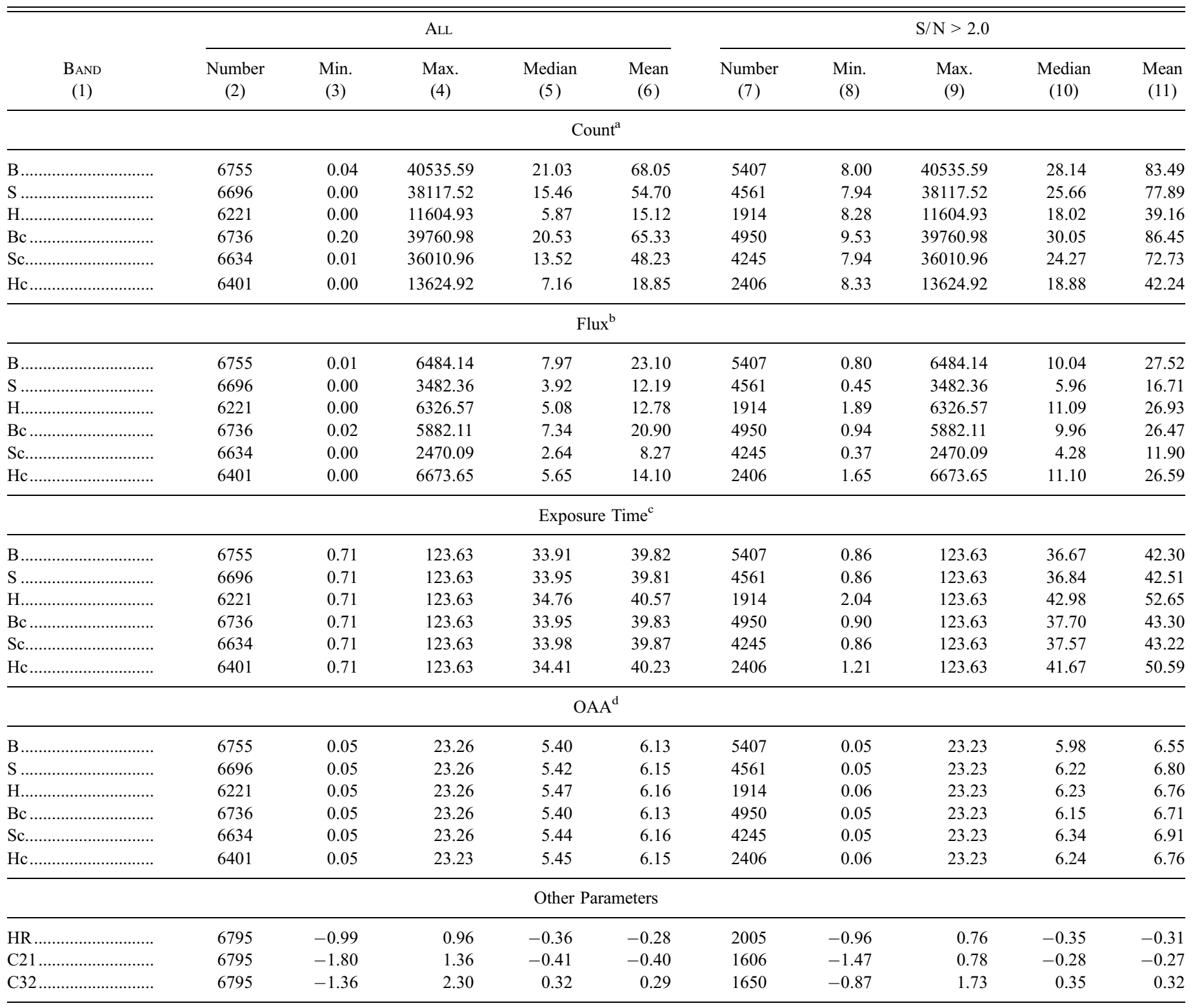

Notes.—Col. (1): X-ray energy band. Cols. (2) -(6): Statistical properties of all ChaMP X-ray point sources. Cols. (7)-(11): Statistical properties of sources with S/N > 2.0.

${ }^{a}$ Source counts.

${ }^{\mathrm{b}}$ Source flux with $\Gamma_{\mathrm{ph}}=1.7$ in units of $1 \times 10^{-15} \mathrm{erg} \mathrm{cm}^{-2} \mathrm{~s}^{-1}$.

c Effective exposure time in units of ks.

$\mathrm{d}$ Off-axis angle in units of arcminutes.

\section{SUMMARY AND CONCLUSIONS}

1. We present the full ChaMP X-ray point source catalog. The main catalog contains 6512 sources from 130 ChaMP fields, and the supplementary catalog contains 853 sources from 19 ChaMP fields that are partly overlapping in their field of view with those in the main catalog. After eliminating duplications, our catalogs contain $\sim 6800$ individual point sources, in a sky area of $\sim 10 \mathrm{deg}^{2}$.

2. The ChaMP X-ray sources are uniformly reduced with the ChaMP XPIPE pipeline and carefully confirmed by visual inspections. Photometry in eight X-ray energy bands, hardness ratio, and $\mathrm{X}$-ray colors of ChaMP X-ray point sources are provided. To calculate the flux of ChaMP X-ray point sources, we also provide the energy conversion factors (ECFs) for each CCD chip and obser- vation for photon indices, $\Gamma_{\mathrm{ph}}=1.2,1.4$, and 1.7, and Galactic absorption, $N_{\mathrm{H}}$, and the vignetting-corrected effective exposure times.

3. To understand the sensitivity and reliability of ChaMP $\mathrm{X}$-ray point sources, we have performed extensive simulations. The detection probability of ChaMP sources is greater than $95 \%$ for source counts of $\gtrsim 30$ and off-axis angle of $<5^{\prime}$. The count recovery rate is $96 \% \pm 1 \%$ regardless of source counts. The wavdetect tool tends to underestimate the net counts for faint sources ( $87 \%$ for $\$ 50$ counts). The false source detection probability is $\sim 1 \%$ of the total detected sources, and $\sim 80 \%$ of these have source counts of $\lesssim 30$.

4. Empirical equations for the positional uncertainties were derived from the ChaMP simulations. The positional uncertainty in ChaMP fields exponentially increases with off-axis angle and 
decreases as the source counts increase with a power-law form. Background fluctuations do not affect the positional uncertainty in our ChaMP sample. The $68 \%, 90 \%$, and $95 \%$ confidence levels of equations for the positional uncertainties are provided.

5. The absolute positional accuracy of the ChaMP X-ray sources is $0.7^{\prime \prime} \pm 0.4^{\prime \prime}$, estimated by matching with SDSS optical sources.

6. The typical ChaMP X-ray point source in the $0.5-2 \mathrm{keV}$ band has counts of 24.3, flux of $4.3 \times 10^{-15} \mathrm{erg} \mathrm{cm}^{-2} \mathrm{~s}^{-1}$, effective exposure time of $37.6 \mathrm{ks}$, and off-axis angle of $6.3^{\prime}$. In the $2-8 \mathrm{keV}$ band, the typical source has counts of 18.9 , flux of $11.1 \times 10^{-15} \mathrm{erg} \mathrm{cm}^{-2} \mathrm{~s}^{-1}$, effective exposure time of $41.7 \mathrm{ks}$, and off-axis angle of $6.24^{\prime}$.

7. The hardness ratio and X-ray colors were calculated with a Bayesian approach that models the detected counts as a Poisson distribution. The typical hardness ratio of the ChaMP X-ray source is -0.35 , and X-ray colors C21 and C32 are -0.29 and 0.36 , respectively.

8. The flux levels (in ergs $\mathrm{cm}^{-2} \mathrm{~s}^{-1}$ ) of sources are $9.4 \times$ $10^{-16}-5.9 \times 10^{-11}(0.5-8 \mathrm{keV}), 3.7 \times 10^{-16}-2.5 \times 10^{-11}(0.5-$ $2 \mathrm{keV})$, and $1.7 \times 10^{-15}-6.7 \times 10^{-11}(2-8 \mathrm{keV})$, respectively. In the X-ray color-color diagram, typical ChaMP sources are located within absorption range $10^{20} \mathrm{~cm}^{-2} \lesssim N_{\mathrm{H}} \lesssim 10^{21} \mathrm{~cm}^{-2}$ and within photon index range $1 \lesssim \Gamma_{\mathrm{ph}} \lesssim 2.5$.

We gratefully acknowledge support for this project under NASA CXC archival research grant AR4-5017X and AR67020X. P. J. G., D. W. K., H. T., and B. J. W. also acknowledge support through NASA contract NAS8-03060 (CXC). M. G. L. is in part supported by the KOSEF grant (R01-2004-000-10490-0).

\section{Facilities: CXO (ACIS)}

Alexander, D. M., et al. 2003, AJ, 126, 539

Barkhouse, W. A., et al. 2006, ApJ, 645, 955

Basilakos, S., Plionis, M., Georgakakis, A., \& Georgantopoulos, I. 2005, MNRAS, 356, 183

Bauer, F. E., Alexander, D. M., Brandt, W. N., Schneider, D. P., Treister, E., Hornschemeier, A. E., \& Garmire, G. P. 2004, AJ, 128, 2048

Brandt, W. N., \& Hasinger, G. 2005, ARA\&A, 43, 827

Brandt, W. N., et al. 2001, AJ, 122, 2810

Chiappetti, L., et al. 2005, A\&A, 439, 413

Gehrels, N. 1986, ApJ, 303, 336

Giacconi, R., Gursky, H., Paolini, F. R., \& Rossi, B. B. 1962, Phys. Rev. Lett., 9, 439

Giacconi, R., et al. 2001, ApJ, 551, 624

Green, P. J., et al. 2004, ApJS, 150, 43

Freeman, P. E., Kashyap, V., Rosner, R., \& Lamb, D. Q. 2002, ApJS, 138, 185

Kim, D.-W., \& Fabbiano, G. 2003, ApJ, 586, 826

Kim, D.-W., et al. 2004a, ApJS, 150, 19 (Paper I)

2004b, ApJ, 600, 59

2006a, ApJ, 644, 829

Kim, M., et al. 2006b, ApJ, submitted

\section{REFERENCES}

Manners, J. C., et al. 2003, MNRAS, 343, 293

Martini, P., Kelson, D. D., Kim, E., Mulchaey, J. S., \& Athey, A. A. 2006, ApJ, 644,116

Murray, S. S., et al. 2005, ApJS, 161, 1

Park, T., Kashyap, V. L., Siemiginowska, A., van Dyk, D. A., Zezas, A., Heinke, C., \& Wargelin, B. J. 2006, ApJ, 652, 610

Pierre, M., et al. 2004, J. Cosmology Astropart. Phys., 9, 11

Rosati, P., et al. 2002, ApJ, 566, 667

Silverman, J. D., et al. 2005a, ApJ, 624, 630 2005b, ApJ, 618, 123

Stark, A. A., et al. 1992, ApJS, 79, 77

Tozzi, P., et al. 2001, ApJ, 562, 42 2006, A\&A, 451, 457

van Dyk, D. A., Park, T., Kashyap, V. L., \& Zezas, A. 2004, BAAS, 36, 933

Vikhlinin, A., Forman, W., Jones, C., \& Murray, S. S. 1995, ApJ, 451, 542

Worsley, M. A., Fabian, A. C., Barcons, X., Mateos, S., Hasinger, G., \& Brunner, H. 2004, MNRAS, 352, L28

Worsley, M. A., et al. 2005, MNRAS, 357, 1281

Yang, Y., Mushotzky, R. F., Steffan, A. T., Barger, A. J., \& Cowie, L. L. 2004, AJ, 128, 1501 